\title{
ABSTRACTS COLLECTION ACNP 59th Annual Meeting: Author Index
}

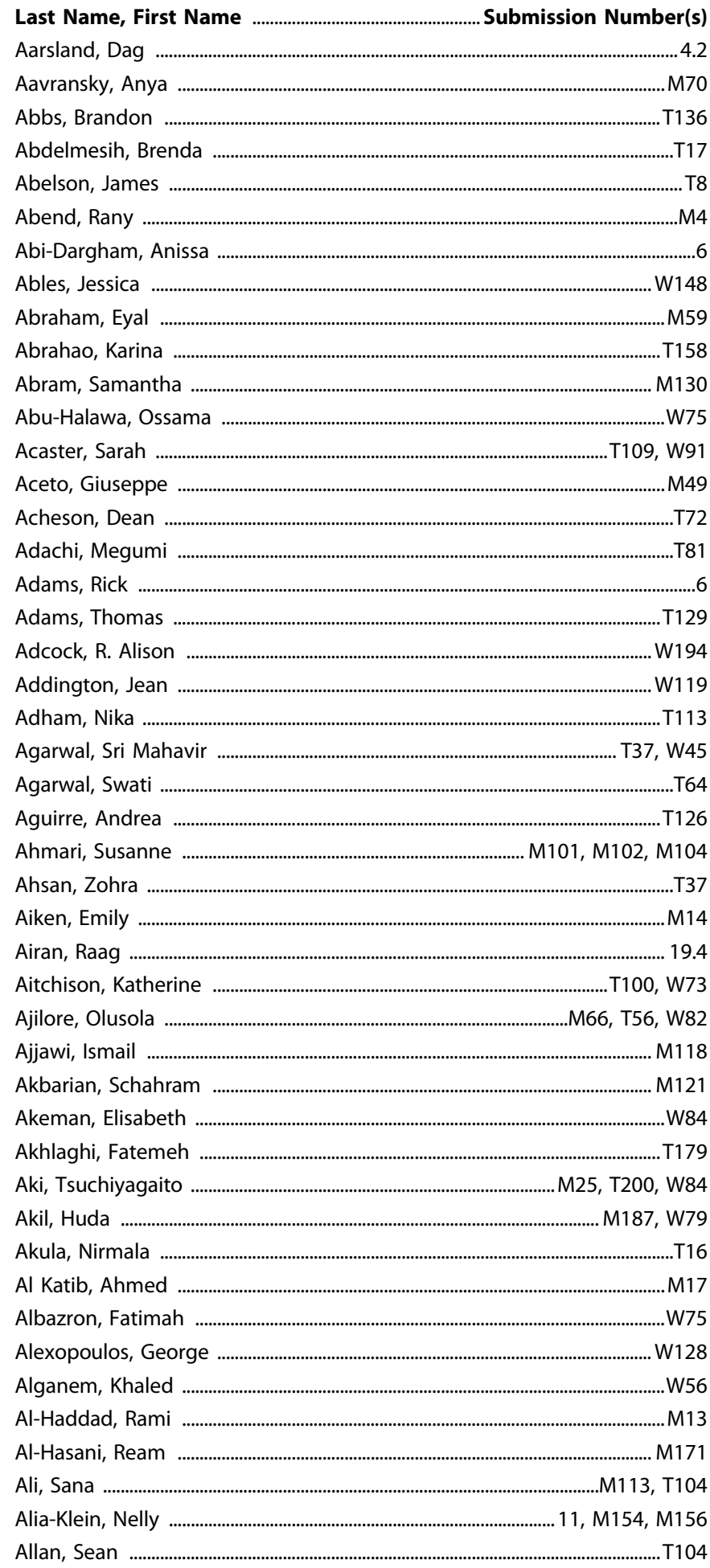

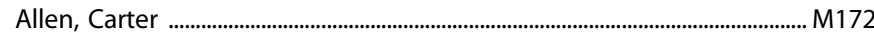

Allene, Camille .......................................................................................................... T126

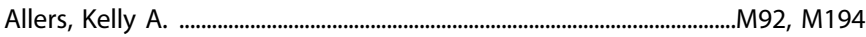

Alleyne, Kiarra ................................................................................................................W27

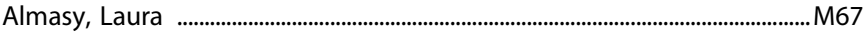

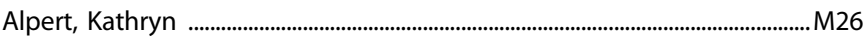

Alpert, Nathaniel M. .................................................................................................... M63

Alphs, Larry ...........................................................................................W110, W118

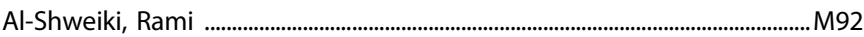

Altar, C. Anthony ........................................................................................................M69

Althaus, Alison ......................................................................................................... T190

Aluri, Jagadeesh .............................................................................................M139, T157

Amara, Susan ......................................................................................................................... W6

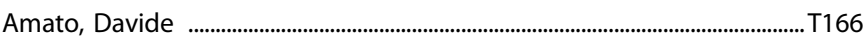

Ameis, Stephanie .........................................................................................M21, T197

Ament, Seth ...................................................................................................

Amodeo, Dionisio ......................................................................................................W200

Anacker, Christoph .......................................................................................M59, W29

Anagnostou, Evdokia ............................................................................................. W101

Ananth, Mala ..................................................................................................................... W3

Ancoli-Israel, Sonia ................................................................................................ T152

Andari, Elissar ................................................................................................................W26

Andersen, Susan .............................................................................................................W38

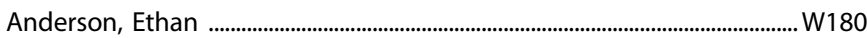

Anderson, Jon Mikael ...............................................................................M191, W171

Anderson, Robyn ...................................................................................................T17

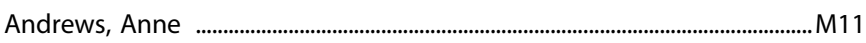

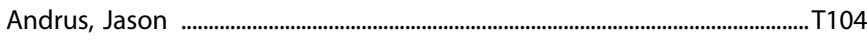

Ang, Yuen-Siang ........................................................................................................... 66

Angarita-Africano, Gustavo ....................................... M165, M191, T7, T45, W171

Angelis, Ariana ..............................................................................................144, T166

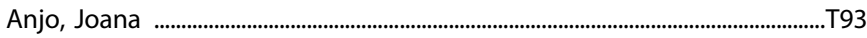

Anthony, Thomas …………………………………………………………………...T144

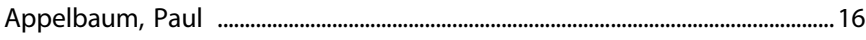

Applebey, Sarah …………………………………………………………....M142, T196

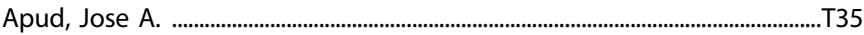

Aquino-Miranda, Guillermo .....................................................................................W20

Arango, Celso ...................................................................................................... 17, T136

Araque, Alfonso ..........................................................................................................T51

Arasappan, Dhivya ...........................................................................................................T16

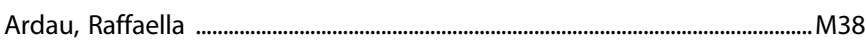

Ardekani, Cory ........................................................................................................... T178

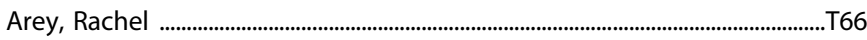

Arfken, Cynthia ........................................................................................................W8 W

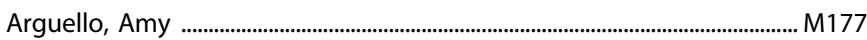

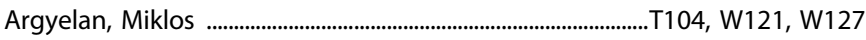

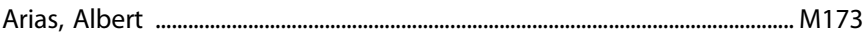




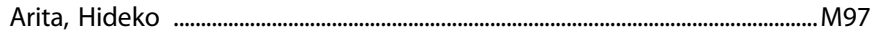

Armand, Sophia

W77

Armstrong, Kristan

M116, M117, T154

Armstrong, Michael W106

Arnow, Bruce .... T5

Arriaza, Jen

$\mathrm{M} 22, \mathrm{~T} 137$

Arruda-Carvalho, Maithe

W30

Artes, Antonio

T159, W43

Arthur, Patrick M17

Arulpragasam, Amanda

W193

Arvidsson, Albin

W77

Arzberger, Thoma

M71

Arzedi, Carlo

M38

Asaoka, Yui

M47

Asch, Ruth

W197

Asci, Irem

... W4

Ash, Peter

T174

Ashford, Elizabeth

W101

Ashkan, Keyoumars W83

Askins, Laura

W5

Aslan, Mihaela

1140

Assaf, Michal

Assali, Ahlem

Aston-Jones, Gary

49.3

Atkins, Alex

M126

Atkinson, Jo-an

M199

Auerbach, Randy

M61

Augustynski, Agatha

T165

Aupperle, Robin

125, T61, W84

Autry, Anita

...T17

Avery, Suzanne

M117

Ayala, Alfred

...T90

Ayub, Rafi ..

W39

Azab, Abed

M79

Azevedo, Estefania

M43

Azuma, Katsunori

Babbs, Richard K.

M95

Baca, Selena

T174, W161

Baca-Garcia, Enrique W81

Bacci, Alberto

..T159, W43

Bach, Snow

...T126

Bachi, Keren

W102

.T180

Bachman, Peter M ..................................................................................................W119

Bachtell, Ryan ...........................................................................................................W18

Badathala, Anusha .................................................................................................W95

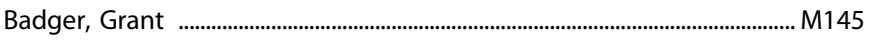

Bae, GiYeul

...T138

Bagot, Rosemary

40, W66

Bai, Yushi

....T48

Bajaj, Thomas

W78

Baker, Dewleen

....T72

Baker, Fiona

...W34

Baker, Krista

M114

Baker, Matthew

W152

Baker, Pamela

W110

Bal, Aneesh

M177, T118
Balana, Bartosz

M89

Baldassarri, Stephen

W171

Bale, Tracy

51, T82

Bali, Purva

W148

Ball, Tali

W67

Ballard, Elizabeth

..T116

Balsam, Peter

M101, W52

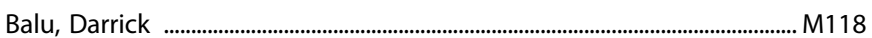

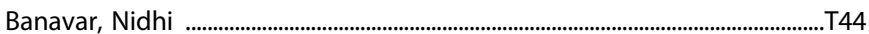

Bangasser, Debra ............................................................................................M48, T178

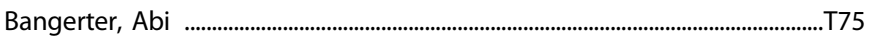

Banihani, Jude ...........................................................................................................T165

Bankole, Kemi ...................................................................................................M80, W92

Bansal, Sonia .............................................................................................................. T138

Baracz, Sarah ........................................................................................................... M161

Barak, Lawrence ......................................................................................................T48

Baram, Tallie Z. ..................................................................................... 34, M162, W28

Baratta, Annalisa ........................................................................................................T160

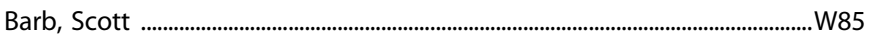

Barbachan e Silva, Mariel ....................................................................................T81

Barbato, Kristiana ....................................................................................................W27

Barber, Anita ..................................................................................................M113, W127

Barch, Deanna ...................................................................................................................... 44

Barkley-Levenson, Amanda .................................................................................W162

Barnes, Samuel .............................................................................................................. M195

Barr, Christina ...................................................................................................................T83

Barr, Justinn ........................................................................................................................ M170

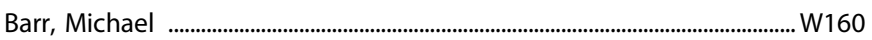

Barrett, Frederick ........................................................................................................T62

Barrow, James .................................................................................... T156, W115

Barry, Brianna ....................................................................................................... W109

Barry, Sarah ................................................................................................................T162

Barson, Jessica ........................................................................................................... T189

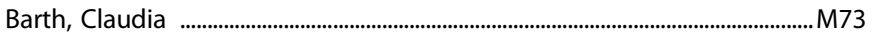

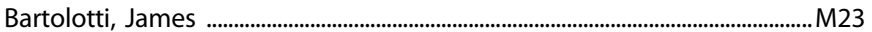

Baruah, Jugajyoti ...................................................................................................... M118

Barzilay, Ran ........................................................................................................... M197

Bassett, Danielle .............................................................................................................T59

Bassetti, Claudio ......................................................................................................... M137

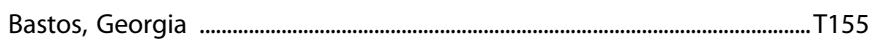

Bateh, Julia ........................................................................................................119, W196

Batra, Kanha .................................................................................................100, T118

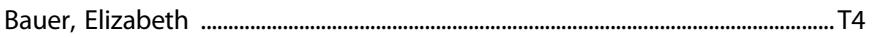

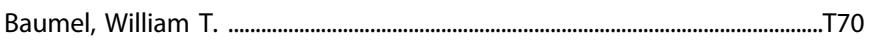

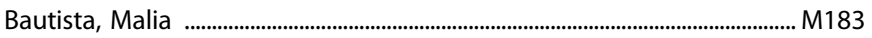

Bavley, Charlotte .....................................................................................................T178

Baxter, Mark .................................................................................................................. W196

Bayshtok, Sabina ...........................................................................................................

Beach, Steven ..........................................................................................................T10

Bearden, Carrie E. ..................................................................................... 8.1, W119

Beardsley, Patrick M. ....................................................................................................... T149

Beardwood, Joy ........................................................................................................T165

Beaumont, Kristin ......................................................................................................W148

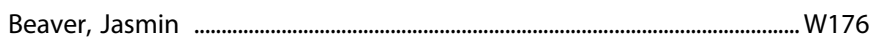

Becker, Georg A. ......................................................................................................M73

Becker, Howard .......................................................................................... 7, 7.2, W180 
Becker, Jill

$3, \mathrm{~T} 193$

Beckmann, Christian

M105, W65

Beckmann, Joshua

M166

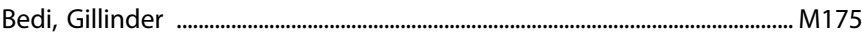

Beerman, Adam ....................................................................................................W138

Behroozi Asl, Bahareh .............................................................................. T100

Beierle, Jacob A.

T174, W161

Belger, Aysenil

.... W119

Bell, Richard

M178

Belleau, Emily L.

M61, M63

Bellon, Alfredo

W108

Belmaker, Robert

...M79

Beloate, Lauren

M150

Beltran, Jacqueline ............................................................................................... T175

Bendl, Jaroslav ......................................................................................................... M121

Benn, Milán .................................................................................................................T190

Benningfield, Margaret M. .......................................................................................T5

Benster, Lindsay ........................................................................................................... M198

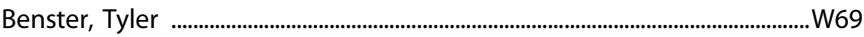

Bentzley, Brandon .................................................................................................... M162

Benveniste, Helene ................................................................................................W151

Bergman, Jack ....................................................................................................... M196

Berlow, Yosef ..............................................................................................................W86

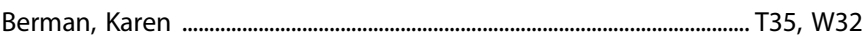

Bernanke, Alyssa .........................................................................................................M44

Berner, Laura ................................................................................................................T8

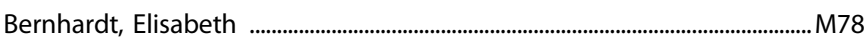

Bernstein, Hans-Gert ..................................................................................................M74

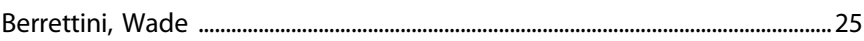

Berry-Kravis, Elizabeth ..........................................................................................W23

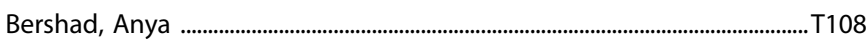

Bersudsky, Yuly ................................................................................................................

Bertherat, Feodora .................................................................................................. M148

Bertholomey, Megan ...………………………………………………………....W146

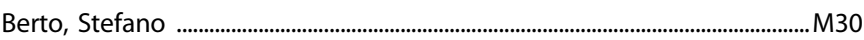

Bertocci, Michele ............................................................................................................ 38.4

Besheer, Joyce ........................................................................................................W13

Besich, Zach ..................................................................................................................W109

Betzel, Richard ..............................................................................................................T59

Beurmann, Silvia ...................................................................................................W112

Beversdorf, David ..........................................................................................................W37

Beyeler, Anna ...............................................................................................................T118

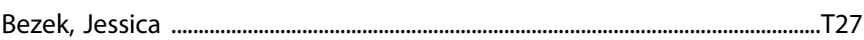

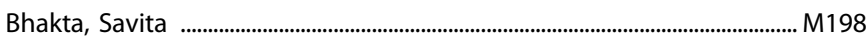

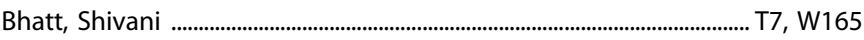

Bhattacharya, Anindya .............................................................................. M88, M89

Bhavna, Solanki ...........................................................................................................W72

Bhupathi, Vasudev ................................................................................................. M1 11

Bi, Guo-hua .................................................................................................................T50

Bidwell, L. Clnnamon ......................................................................................... M174

Biernacki, Kathryn ................................................................................................W153

Biesecker, Catherine ...................................................... M159, W151, W156, W173

Bigdeli, Tim ................................................................................................................... T140

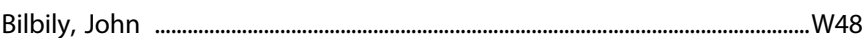

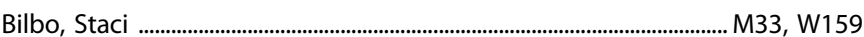

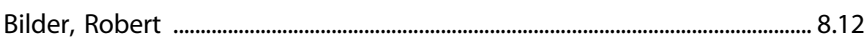

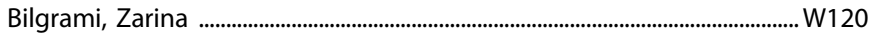

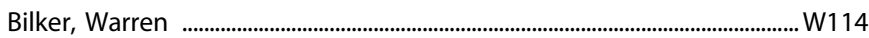

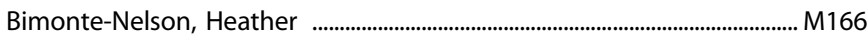

Binder, Elisabeth ...........................................................................................................M71

Bingham, Kathleen ............................................................................................W12

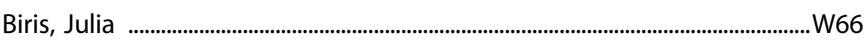

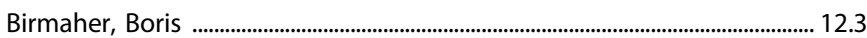

Birnbaum, Michael ........................................................................................M113, W127

Birnbaum, Rebecca ................................................................................................ M120

Birnie, Matthew .............................................................................................. M162, W28

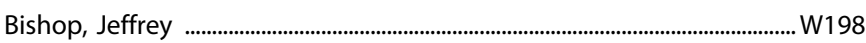

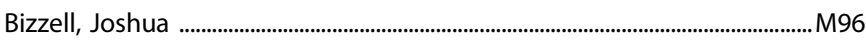

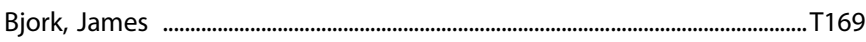

Blackford, Jennifer ............................................................................ M116, T55, T154

Blackman, Rachael ................................................................................................. T35

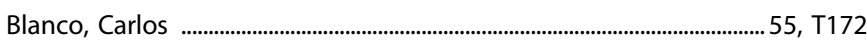

Blazer, Annie ........................................................................................................... M128

Blendy, Julie ....................................................................................................................... 49.2

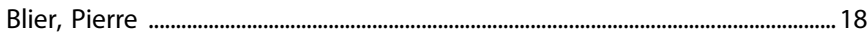

Bloch, Yehudit ........................................................................................................ M79

Blokhin, llya ..........................................................................................................W147

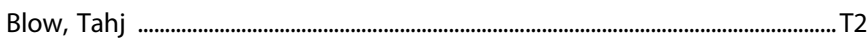

Blumberg, Hilary ......................................................................................................M60

Blumberger, Daniel M. ..........................M21, M93, T25, T92, T133, T153, W107

Boateng, Comfort .......................................................................................................T49

Bobadilla, Ana-Clara ......................................................................M144, W143, W183

Bocchetta, Alberto ....................................................................................................

Bodurka, Jerzy ..........................................................................M25, T73, T200, W84

Boehme, Rebecca ...................................................................................................W172

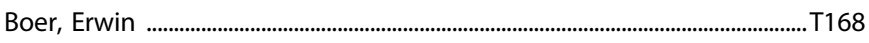

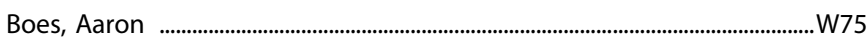

Bogenschutz, Michael .............................................................................................T46

Bohacek, Johannes ........................................................................................................ W8

Bohnsack, John Peyton ...........................................................................M149, T164

Boland, Elaine ................................................................................................................T97

Boley, Angela ........................................................................................................................ M136

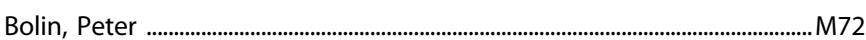

Bollinger, Justin ............................................................................................................. T12

Bolton, James .................................................................................................................W42

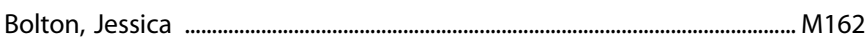

Bonagura, Darla ..........................................................................................................W153

Bonaventure, Pascal ............................................................................... 28, M88, M89

Bondy, Erin ...................................................................................................................M61

Bongiovanni, Angela ...................................................................................... T178

Bonnin, Alexandre .........................................................................................................

Bonthapally, Vijayveer .................................................................................T109, W91

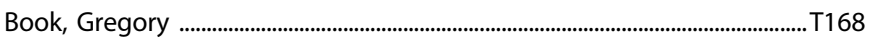

Boomhower, Brent ................................................................................................. M168

Borckardt, Jeffrey .....................................................................................................W170

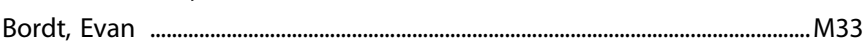

Borentain, Stephane .......................................................................................................... T93

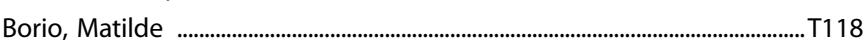

Borkar, Chandrashekhar ............................................................................................W21

Boroda, Elias ................................................................................................................W106

Borrelli, Kristyn ................................................................................................................ W161

Borrie, Sarah ........................................................................................................................ M29 
Bortz, David

Bossert, Jennifer

M155

Bousman, Chad

M142, T196

Bouyssi-Kobar, Marine ..................................................................................................W25

..T100, W73

Bowen, Carrie ....................................................................................................... T149

Box, Adrain ............................................................................................................W73

Boyle, Catherine ......................................................................................................T168

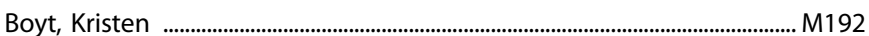

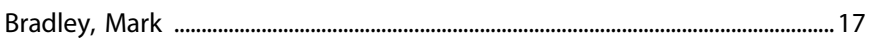

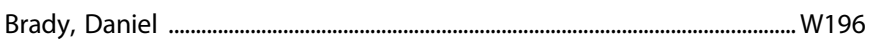

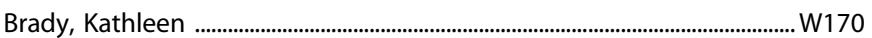

Brady, Lillian .........................................................................................................................

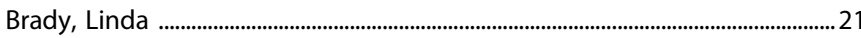

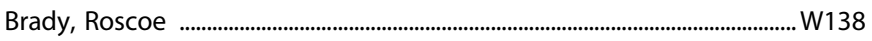

Braff, David .............................................................................................................. M134

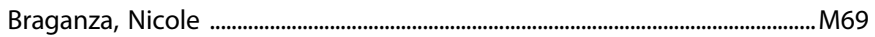

Branchek, Theresa ..........................................................................................................

Brannan, Stephen .......................................................................................21, M110

Bravo-Rivera, Hector ..................................................................................................... M18

Breier, Alan

M110

Breiger, Micah

M195, M196

Breithaupt, Lauren

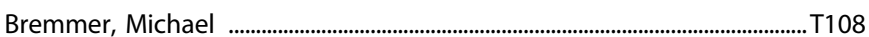

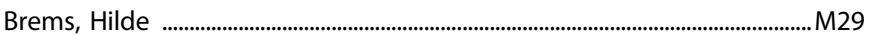

Brenhouse, Heather ...........................................................................................39, W11

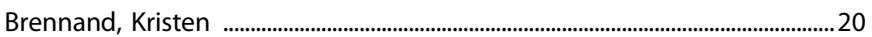

Brenner, Ronald ............................................................................................................ T134

Brewer-Scotti, Heather ..........................................................................................T4

Brian, Kopell .........................................................................................................................W83

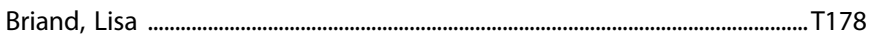

Bridges, Catherine ................................................................................................... M30

Brien, Brittany $\mathrm{O}^{\prime}$.........................................................................................................T106

Briggs, Sherri ......................................................................................................................... M143

Brigman, Jonathan ............................................................................................... M198

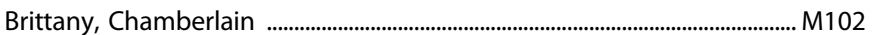

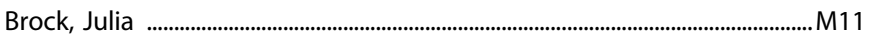

Brooks, Julia ........................................................................................................W36

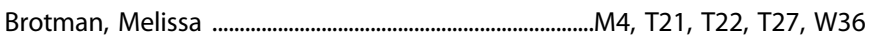

Brown, Brandon ....................................................................................................T74

Brown, E. Sherwood ..................................................................................T117, W68

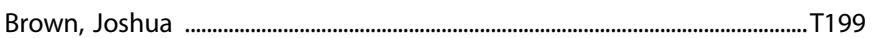

Brown, Krystal ...............................................................................................................W74

Brown, Kyle ............................................................................................................W18

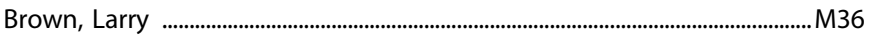

Brown, Lilly .......................................................................................................................... M197

Brown, Natalie ........................................................................................................T163

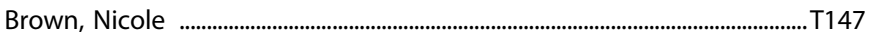

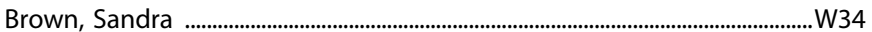

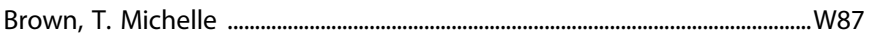

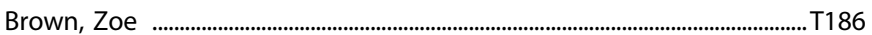

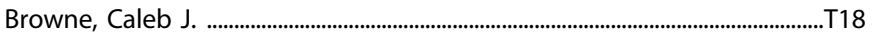

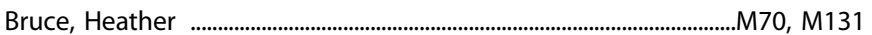

Bruce, Steven ..............................................................................................................W17

Bruchas, Michael .............................................................................................10, T54

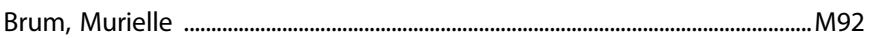

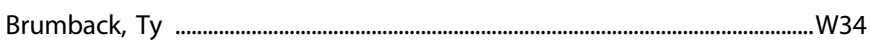

Brunkhorst-Kanaan, Nathalie .................................................................................... M92

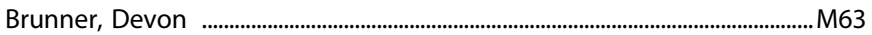

Bruno, Jennifer ............................................................................................................. T13

Bruss, Joel .................................................................................................W75

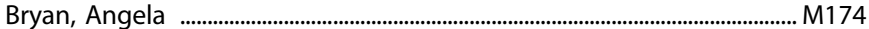

Bryant, Camron D. ......................................................................................T174, W161

Buchanan, Robert .......................................................................................W112, W121

Buchner, Amanda ................................................................................................T100

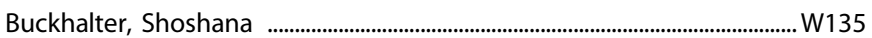

Budac, David .........................................................................................................................W1

Bugarski-Kirola, Dragana ......................................................................................... T136

Bujarski, Spencer ........................................................................................................ M152

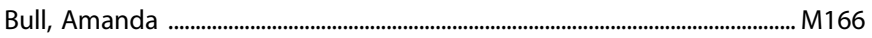

Burdick, Katherine ....................................................................................................... M58

Burghardt, Kyle ........................................................................................................... M19

Burkhouse, Katie ...................................................................................................... M158

Burns, Chloe ........................................................................................................... M94

Busler, Jessica ..........................................................................................................T67

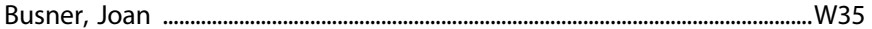

Bustillo, Juan .........................................................................................M124, M130

Byrd, Nia ............................................................................................................ T183

Caballero, Camila ...............................................................................................................

Caceda, Ricardo ……………………………………………………………………...T99

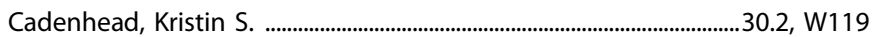

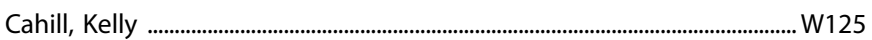

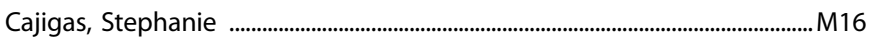

Calabrese, Joseph .........................................................................................................T110

Calabrese, Mauro ………………………………………………………………..... M169

Calakos, Katina .............................................................................................................. W171

Calakos, Nicole ........................................................................................................... 48.4

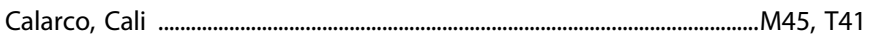

Calhoon, Gwendolyn ................................................................................................T118

Calhoun, Vince ...................................................................42, 42.2, M122, M130

Caligiuri, Michael ............................................................................................................ M125

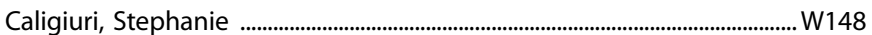

Calipari, Erin ..............................................................M16, M185, M186, M190, W167

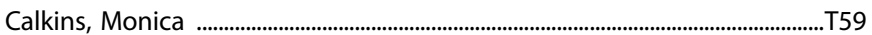

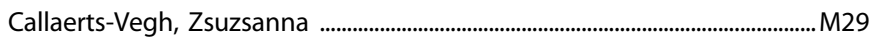

Camchong, Jazmin .....................................................................................................T78

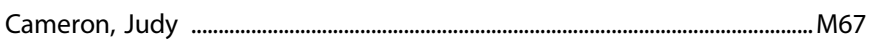

Cameron, Michael .......................................................................................................... M143

Campaña-Montes, Juan José ....................................................................T159, W43

Campbell, Erin ............................................................................................................... M176

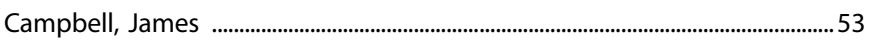

Campbell, Rianne ...................................................................................................T165

Campbell, Una .........................................................................................................T149

Campus, Paolo .................................................................................................................... M187

Canetta, Sarah ..................................................................................................... 24.2

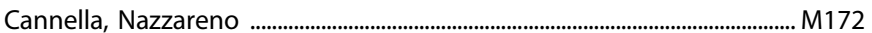

Cannon, Tyrone ................................................................................... 21, T142, W119

Canovali, Gia ............................................................................................................W8

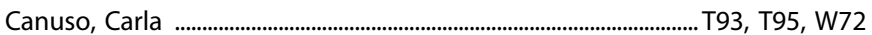

Caplin, Phoebe …………………………………………………………………….... T111

Caradonna, Salvatore .................................................................................................W79

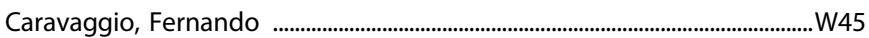

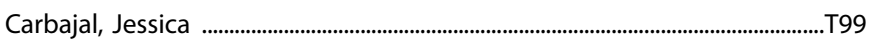


Cardenas, Anjelica

Cardenas, Emili

Cardinale, Elise

Cardoso, Laura

M134

Carelli, Regina

M160

Caria, Paola

Carino, Kathleen

Carla, Yuede

Carlezon, William

Caro, Keaven

Caron, Marc

Carpenter, Linda

Carpenter, Rachel

Carpiniello, Bernardo

Carpio, MJ

Carr, Gregory

Carr, Michael

Carrette, Lieselot

Carrion, Ricardo E

Carrubba, Erin

Carson, Richard

Carter, Cameron

Carter, Jordan

Carter, Sierra

Carvalho Henriques, Beatriz

Cassidy, Clifford

Castillo, Monica Feliz

Castillo-Passi, Rolando

Castro, Daniel

Catallini, Joseph

Cattaneo, Annamaria

Cavalco, Natalie

Cavanagh, James

Cavanagh, Jonathan

Cavanna, Federico

Cavus, Idil

Cayton, Christelle

Ceceli, Ahmet

Cecil, Kim

Celebi, Seyda

Cenik, Basar

Cenik, Can

Centanni, Samuel

Cereceo Page, Stephanie ...........................................................................................M20

Cerfolio, Nina

..T139

Cernasov, Paul M.

....M96

Cesnaite, Elena

Cha, Jiook

Chahine, George

Chakravarty, Mallar

Chalhoub, Reda W183

Chamberlin, Linda

Chan, Shi Yu M123

Chandra, Ramesh ....T41

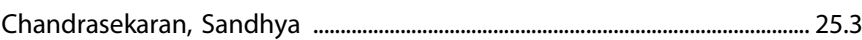

Chang, Fini
Chang, Lijia

M77, T115

Chang, Linda

Chappell, Amy

.. .53

Charlet, Katrin

T176

Charney, Dennis ............. 4.3

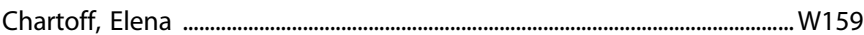

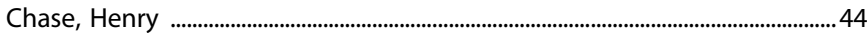

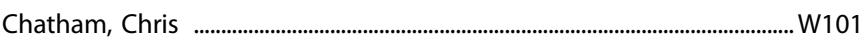

Chatzinakos, Christos ..............................................................................................W78

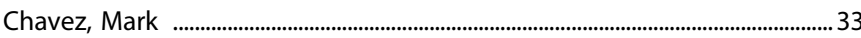

Chawla, Sumedha .................................................................................................... T183

Cheer, Joseph ...........................................................................................180, M188

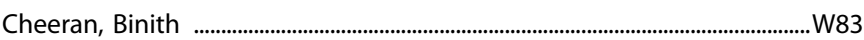

Chen, Briana K. ......................................................................................................M6

Chen, Chao ............................................................................................................... T141

Chen, Haiying ................................................................................................................. M49

Chen, Hannah ................................................................................................................ T9

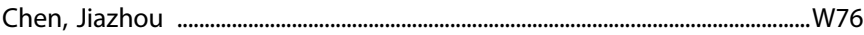

Chen, Joanna ..........................................................................................................T8

Chen, Melanie M. .......................................................................................... T174, W161

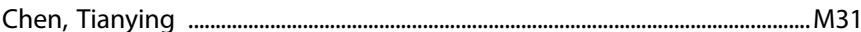

Chen, Ying ........................................................................................................... M149

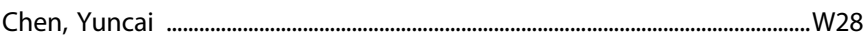

Chen, Zexin ............................................................................................................. M100

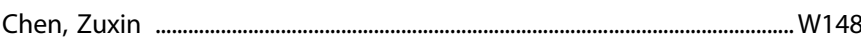

Cheng, Lesley ....................................................................................................................... $\mathrm{T} 142$

Chesler, Elissa ..................................................................................................................W163

Chetkovich, Dane ........................................................................................................M50

Chew, Jennifer ..........................................................................................................W109

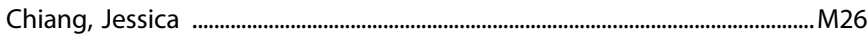

Chiappelli, Joshua ...........................................................................M46, M70, M131

Childress, Anna Rose ..............................................................................M164, W155

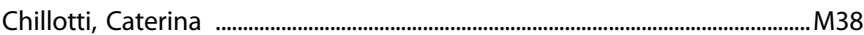

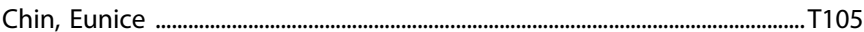

Chin Fatt, Cherise ...........................................................................................T68, W199

Chioma, Vivian .......................................................................................M144, M150

Chitayat, David ............................................................................................................ T111

Chitnis, Om .............................................................................................................. T165

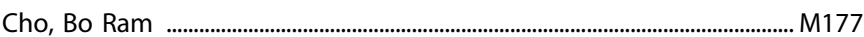

Cho, Christina .............................................................................................................W196

Cho, Kang-lk K. ...................................................................................................W137

Chohan, Muhammad ........................................................................................... M101

Choi, Doo-Sup ................................................................................W41, W139, W152

Choi, Hye Sun ...............................................................................................................T74

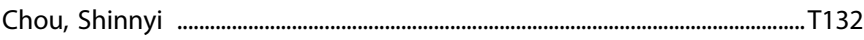

Choudhary, Vikash ...................................................................................................T126

Choudhury, Sourav ......................................................................................................T118

Chowdari, Kodavali .................................................................................................W163

Chowdhury, Tara ........................................................................................................... 27.2

Christensen, Michael Cronquist .............................................................................. W126

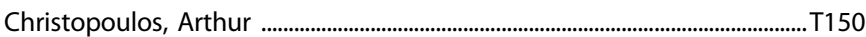

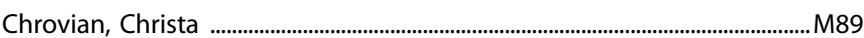

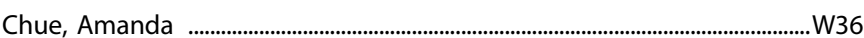

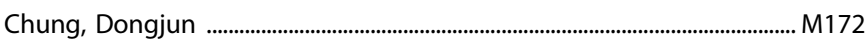

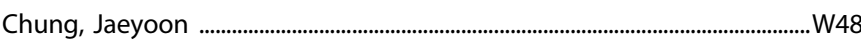

Church, Taylor ..................................................................................................................... T111 
Ciccocioppo, Roberto

M172

Cifani, Carlo

M142, T196

Ciric, Rastko

....T59

Cirrito, John

M171

Cisler, Joshua

M12

Cissé, Yasmine-marie ...........T82

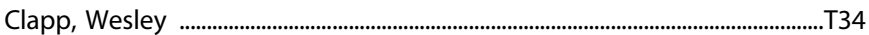

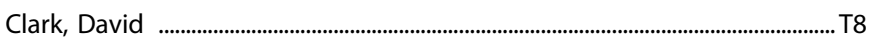

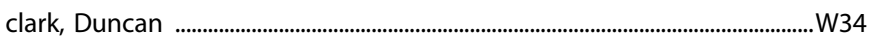

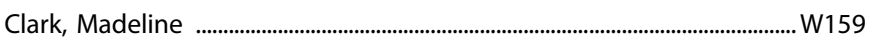

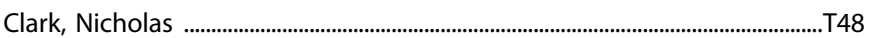

Clarke, Gerard ...........................................................................................................W89

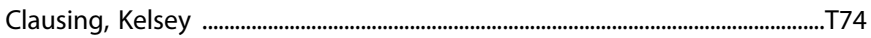

Clauss, Nikki ......................................................................................................... M147

Claxton, Amy

M125

Clem, Roger

M119, W18

Clementz, Brett

W198

Clinch, Susanne

W101

Coccaro, Emil

11

Cocco, Cristina

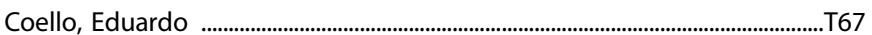

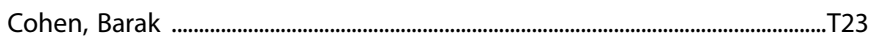

Cohen, Johnathan ............................................................................................................W64

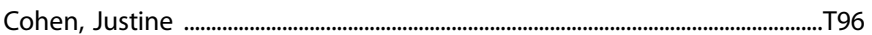

Cohen, Lee ........................................................................................................................... T111

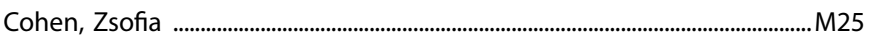

Cohn, Tony .......................................................................................................................T37

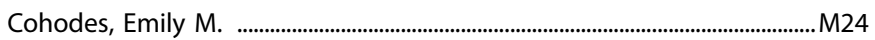

Cole, Anthony .....................................................................................................................W99

Cole, Robert .......................................................................................................... W140

Cole, Shannon ............................................................................................................ M180

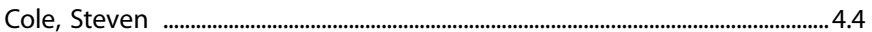

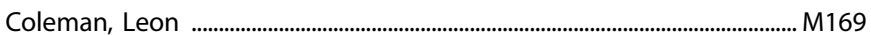

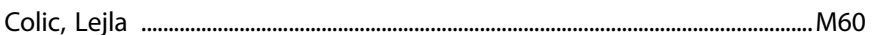

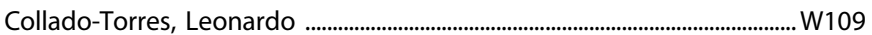

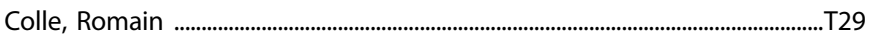

Collins, Gregory ......................................................................................................W175

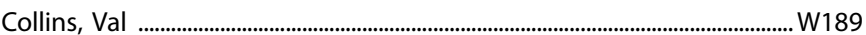

Compere, Laurie ........................................................................................................W8

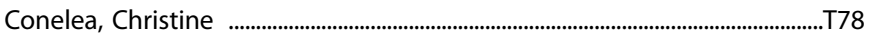

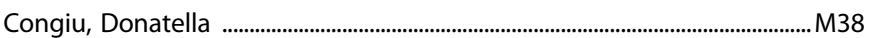

Conklin, Cynthia .........................................................................................................W154

Conn, P. Jeffrey .............................................................................................................. 48

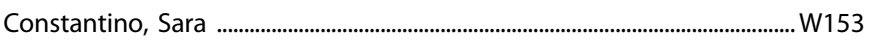

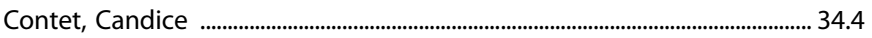

Conway, Charles ........................................................................................................W74

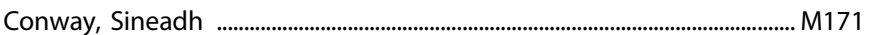

Cook, Phillip ........................................................................................................... T59

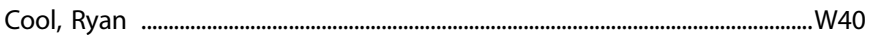

Cooper, Jessica ........................................................................................................... W193

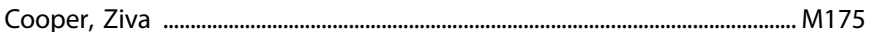

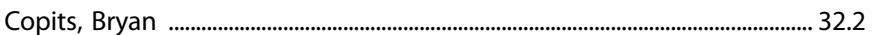

Corcoran, Cheryl ................................................................................................. 8 , W120

Corkrum, Michelle ............................................................................................M35, T51

Corlett, Philip

Cornblatt, Barbara A

... W119
Cornblatt, Brian

$\mathrm{M} 22, \mathrm{~T} 137$

Cornier, Marc-Andre

....W46

Cornish, Jennifer

M161

Corriveau, Taryn J.

..W96

Corruble, Emmanuelle ................................... T29

Cortez-Resendiz, Alonso ................................................................................W10 W

Corvin, Aiden ........................................................................................................W102

Cosgrove, Kelly ....................................M165, M191, T7, T45, W157, W165, W171

Cosmo, Camila .....................................................................................................................14

Cossio, Daniela ......................................................................................................W140

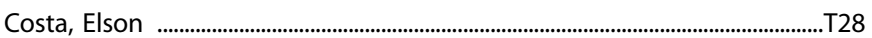

Costa, Vincent .............................................................................................T22, W192

Cotton, Andrew ...............................................................................................................W26

Cottone, Pietro ...........................................................................................................T161

Covault, Jonathan ...................................................................................................... M173

Cover, Kara ...............................................................................................................W190

Cowan, Christopher …………………………….....M30, T162, W55, W145, W180

Cox, Sylvia ......................................................................................................................W18

Crane, Natania ................................................................................................................... M158

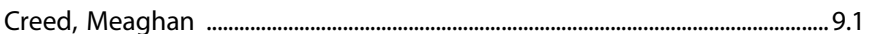

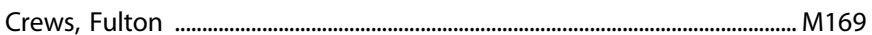

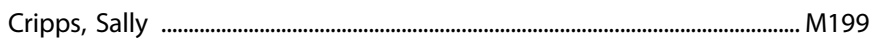

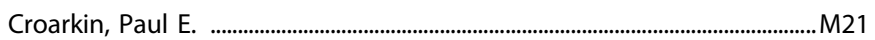

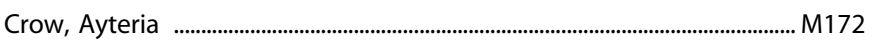

Crowell, Andrea ........................................................................................................W8

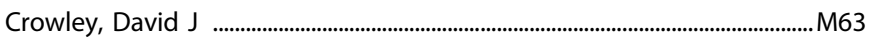

Cruz-Martin, Alberto ................................................................................................W161

Cruz-Sanchez, Arely ..................................................................................................W30

Cubells, Joseph ..........................................................................................................W26

Cuerdo, Arlene .......................................................................................................... M114

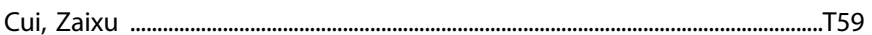

Culkin, Hannah ..............................................................................................................T71

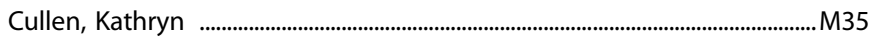

Cullum, C. Munro …………………………………………………………………..... M78

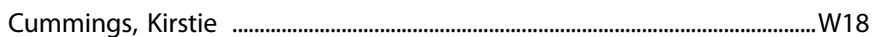

Cummings, Richard .......................................................................................................T T36

Cunningham, Jacobi ............................................................................................. T151

Curley, James .............................................................................................................. M100

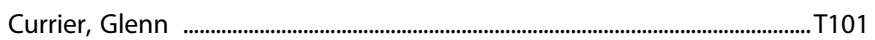

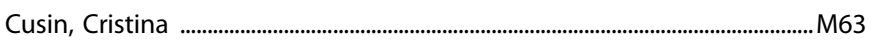

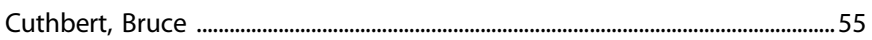

Cvetkovska, Vedrana ...............................................................................................W66

Cyr, Marilyn .........................................................................................................T31, T128

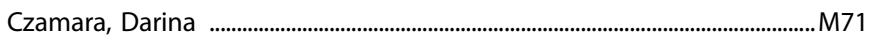

Czarnecki, Kyle .............................................................................................................. W7

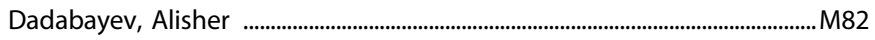

Dagher, Alain .................................................................................................................. W18

Dagher, Merel ............................................................................................................................ M11

D'Agostino, Anthony ................................................................................................W83

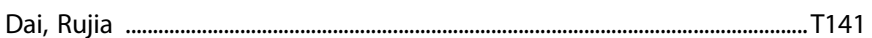

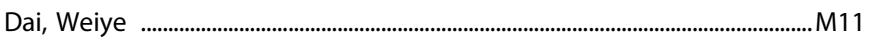

Daly, Rebecca ....................................................................................................W130

D'Amour-Horvat, Valérie ....................................................................................W186

Daniel, David G. ..........................................................................................................W35

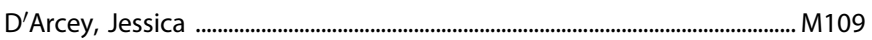

Darnley, Stefanie ........................................................................................................... M164 
Darwish, Mona

Das, Ravi

das Gupta, Rebecca

D'Ascenzo, Marcello

Daskalakis, Nikolaos

Daskalakis, Zafiris J. $\mathrm{M} 21, \mathrm{~T} 25, \mathrm{~T} 92, \mathrm{~T} 133, \mathrm{~T} 153, \mathrm{~W} 107$

Davatolhagh, M. Felicia ....T63

Davatzikos, Christos

T59, W114

David, Denis ....T29

Davidow, Juliet

39.3

Davids, Antonette

W27

Davidson, Michael

.....21

Davie, William

...W17

Davies, Paige

...M69

Davis, John M

M22, T137

Davis, Margaret

...W40

Davis, Michael

Davis, Robert

T110, T148

Daws, Lynette

M147, W176

Dax, Scott

....53

De Bellis, Michael ................................................................................................W34

De Boer, Peter ............................................................................................................W60

de Brito van Velze, Marcel ..........................................................................................T126

de Guglielmo, Giordano .......................................................................................... M170

De la Fuente, Laura Alethia ........................................................................................T58

de la Fuente-Sandoval, Camilo ..........................................................................W1 W10

de Laat, Bart ...........................................................................................................................W157

De Landri, Allegra V. ........................................................................................................M6

de Lecea, Luis ....................................................................................................................... 28.1

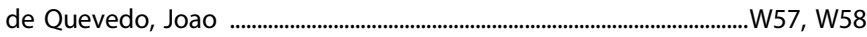

de Wit, Harriet .......................................................................................................... 47, $\mathrm{T} 108$

Deacon, Terrence ........................................................................................................W136

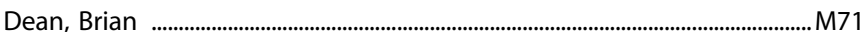

DeBattista, Charles ..................................................................................................W W74

deBettencourt, Megan ...............................................................................................W64

Debonee, Sarah ..............................................................................................................W40

DeBrosse, Adrienne ............................................................................................. T156

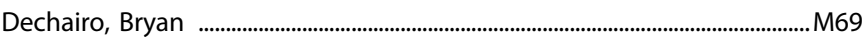

Decker, Amanda ………………………………………………………………….... T112

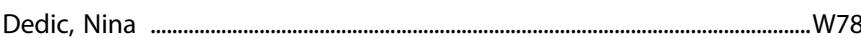

Defaix, Céline .................................................................................................................T29

Deisseroth, Karl ................................................................................................... M187, W69

Del Zompo, Maria ...........................................................................................................M38

Delaney, Christopher ............................................................................................ T102

Delano-Wood, Lisa ......................................................................................................W100

DelBello, Melissa P. ................................................................................... 12.2, T70

Deligiannidis, Kristina M. ....................................................M80, T109, W92, W92

Demaestri, Camila .....................................................................................................W11

Demars, Michael ............................................................................................M119, W196

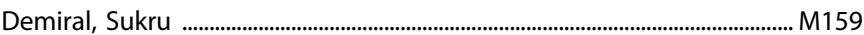

Demitrack, Mark ................................................................................................ T125, W105

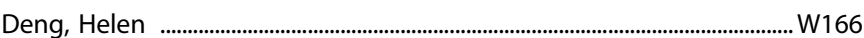

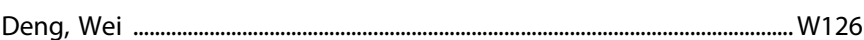

Denney, Thomas .......................................................................................................... T69

Denny, Bryan ...…………………………………………………………………………... W191

Denny, Christine Ann ............................................................... M6, T29, T114, W19
Depalmer, Giuliana .....................................................................................................T129

Depp, Colin .............................................................................................................W132

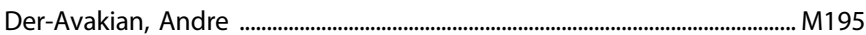

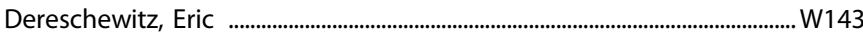

DeRosse, Pamela ..........................................................................113, W121

Desarkar, Pushpal ......................................................................................................M21

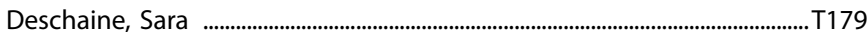

Deshpande, Rangaprakash .................................................................................. M103

Desrochers, Stephanie .......................................................................................W52

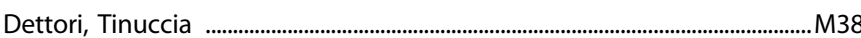

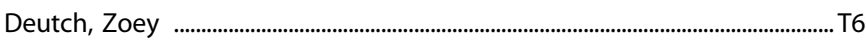

DeVille, Danielle .............................................................................................................W84

Devlin, Bernie ................................................................................................................W124

DeVries, Derek M. ................................................................................................... T162

DeVries, William H. .................................................................................................... T199

Dharanikota, Ayarah ...................................................................................................... M96

D'Hooge, Rudi ............................................................................................................. M29

Diaz, Joel .................................... M103

Diazgranados, Nancy .............................................T172, T183, T195, W151, W156

Dichter, Gabriel S. ................................................................................................M96

Dickerson, Faith ......................................................................................................W113

Dickerson, Kathryn C. ................................................................................................ W194

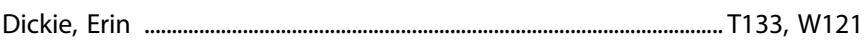

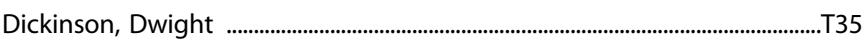

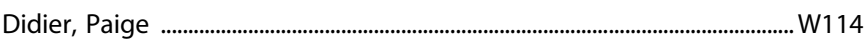

DiDomenico, Grace .................................................................................................... M197

Diedrich, Jolene ...........................................................................................................T39

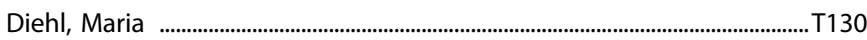

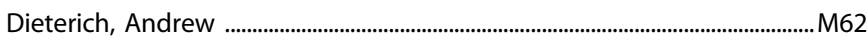

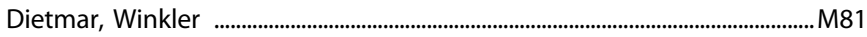

Dietz, David ...............................................................................................................T40

Difeliceantonio, Alexandra ...................................................................................W148

Dillon, Daniel G. ..............................................................................M63, M96, M195

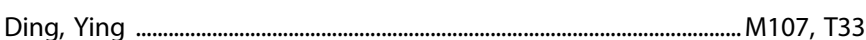

Dingemanse, Jasper ............................................................................................. W1

DiPetrillo, Lauren ............................................................................................. M111

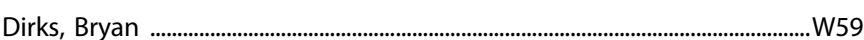

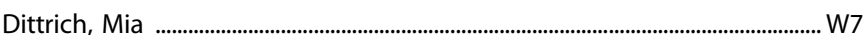

Dixon, Rushell ................................................................................................M59, W29

Do Monte, Fabricio ....................................................................................................W20

Dobbs, Lauren .............................................................................................................. 10.4

Dobrowolny, Henrik ..................................................................................................... M74

Doernberg, Ellen ...........................................................................................................W24

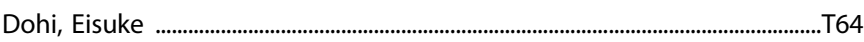

Dolder, Patrick ...................................................................................................................W61

Dombrovski, Alexandre .......................................................................................W76

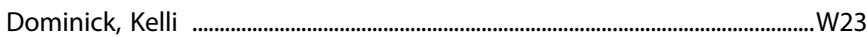

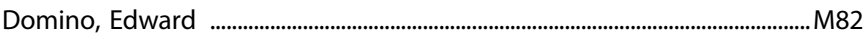

Donaldson, Zoe _................................................................................................T119

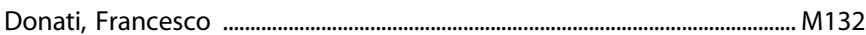

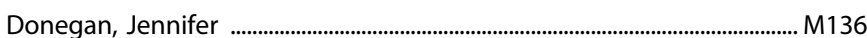

Dong, Pengfei ................................................................................................................ M121

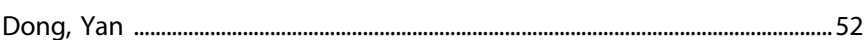

D'Onofrio, Gail ................................................................................................................ 50

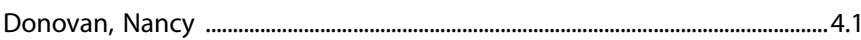

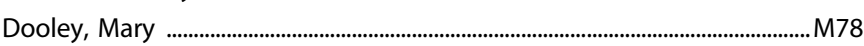


Doolin, Kelly M92

Doucet, Gaelle

W120

Doucette, Kristin

Doucette, Wilder

Dougherty, Joseph

Dowling, Joseph

Downar, Jonathan

Downey, Melissa

Downing, Nancy

Doyle, Alysa

Doyle, Michelle

Drake, Lindsey

Drevets, Wayne

Dror, Ron

Drysdale, Andrew

D'Souza, Deepak

Du, Han

DuBois, Samantha

Duckworth, Kenneth

Duda, Jessica

Dudek, Serena

Dugan, Elizabeth

Dugovic, Christine

Dukes, Angeline

Duncan, Erica

Duncan, Laramie

Duncan, Markus

Duncan, Wallace

Dunlop, Boadie

Dunn, Erin

Dunsmoor, Joseph

Duong, Timothy

Durgam, Suresh

Durham, Katherine

Duss, Sian

Duval, Elizabeth

Dvorak, Nolan M.

Dwiel, Lucas

Dworak, Heather

Dworsky-Fried, Zoe

Dyer, Samya

Dymecki, Susan

Dzyubachyk, Oleh

Ebata, Yuko

Ebdrup, Bjørn

Echevarria-Rivera, Angel

Eck, Samantha

Eckert, Anne

Eckert, William

Eddy, Kamryn

Einhorn, Nathan

Eisenberg, Daniel

Eitan, Renana

El Fakhri, Georges

Elam, Hannah

Elayouby, Karim
T161

W49

T23, T174

M39

....T92

..W90

.... 44

W31

W175

M165

M88, W60, W72

...T150

.... T6

.....16

M152

M114

....21

...T96

W78

.... W1

M88

M183

T146, W119, W129

.........

..T37

T116

.W74

W31

.... T2

M67

T110, T148

M61

... W8

.... $\mathrm{T} 8$

M49

W49

M108

M99

M94

...T91

...T60

M97

42.5

W169

M48

W61

M88

..T15

W79

T35, W32

.W83

M63

W134

W148
Elbekai, Reem .........................................................................................................W5

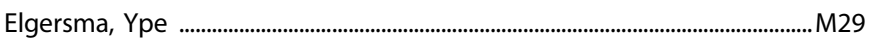

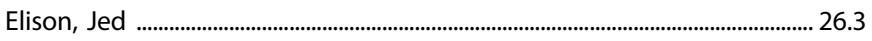

El-Kalliny, Mostafa ..........................................................................................................T119

Ellingson, Benjamin M. ........................................................................W110, W118

Elliott, Mark .....................................................................................................................W114

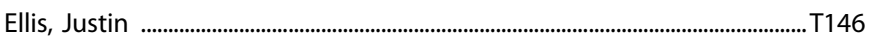

Ellison, Zachary ............................................................................................................ M144

ElSayed, Mohamed ......................................................................................................M17

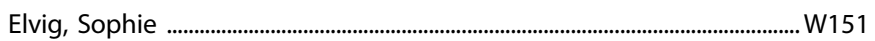

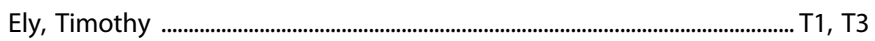

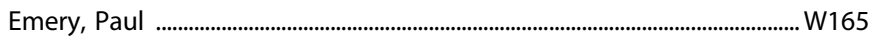

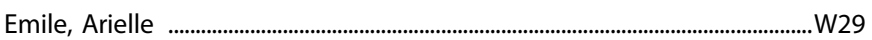

Endo, Nozomi .........................................................................................................

Engelhard, Matthew .................................................................................. M27, W154

Engelke, Douglas ..................................................................................................W2 W

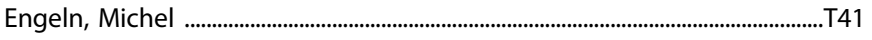

Enomoto, Shingo .........................................................................................................T81

Epperson, C. Neill .....................................................................3.3, M75, M80, W92

Erdmann-Gilman, Petra .......................................................................................... M171

Erickson, Craig ..................................................................................... T11, T80, W23

Erickson, Susan ................................................................................................... M107

Erpelding, Christina ................................................................................................W46

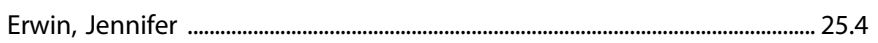

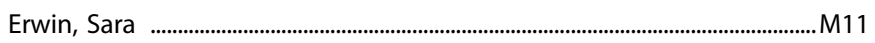

Eshel, Neir ................................................................................................................ $5.2,11$

Esmaeili, Habib .......................................................................................................T94

España, Rodrigo ............................................................................................................T63

Esterlis, Irina ................................................................................................... M191, W40

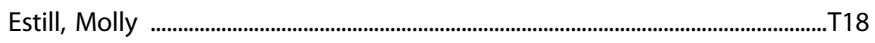

Etkin, Amit ........................................................................................................................... T5

Eto, Kei ...................................................................................................................... M127

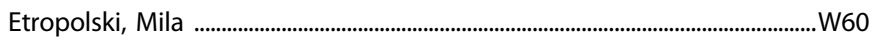

Euston, Tanner ................................................................................................................ M185

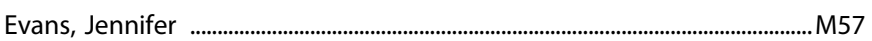

Everett, Nick ................................................................................................................. M161

Eversole, Elizabeth ....................................................................................................W192

Evins, A. Eden ............................................................................................................ T182

Eyler, Lisa .................................................................................................. 1, 31.3, T103

F. Pardiñas, Antonio .............................................................................................W12

Fadok, Jonathan ..........................................................................................................W21

Fahey, Jed ..........................................................................................................M22, T137

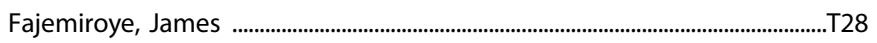

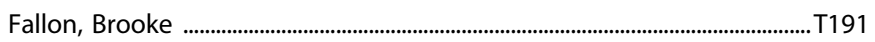

Famularo, Sydney ..............................................................................................T178

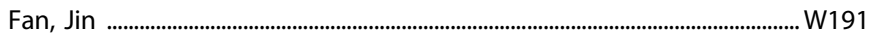

Fan, Mikayla ..................................................................................................................W73

Fang, Hao Shu ........................................................................................................... M100

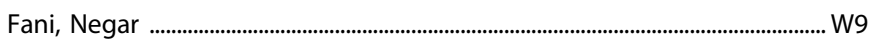

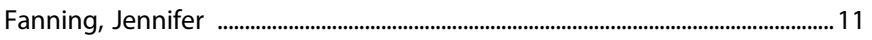

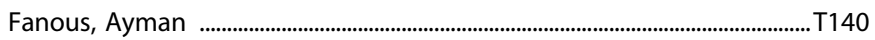

Farmer, Charlene .....................................................................................................T147

Farokhnia, Mehdi ...........................................................................M167, T179, T183

Farrell, Mitchell ........................................................................................................... T170

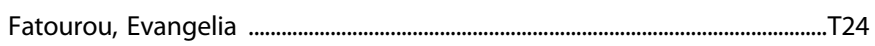

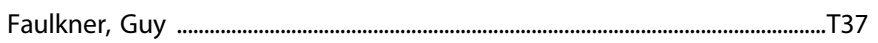


Faulkner, Monica

Fava, Maurizio

... M54, M63, W59

Fayyazuddin, Amir

.......T81

Feather, Abigayle

Federoff, Monica

Feinn, Richard

Feinstein, Justin

Felder, Christian

T150

Feldman, Dana

Felger, Jennifer M159, W151, W156, W173

Felix-Ortiz, Ada

23, 23.5

Fenske, Alicia

...T118

Feola, Brandee

W106

Ferguson, Bradley

M116, T55

Ferguson, Brielle

....W37

Ferguson, Susan

....M40

Fernandes, Brisa

W188

Fernandez-Leon, Jose

M64

Ferrarelli, Fabio

W20

Ferrell, Antanovia ................................................................................................... T155

Ferretti, Arianna .............................................................................................................. T186

Ferretti, Casara ..........................................................................................................W24

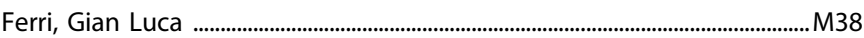

Ferris, Craig ................................................................................................................W11

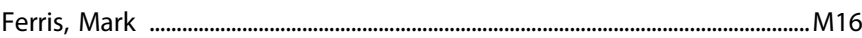

Feurer, Cope .....................................................................................................................T56

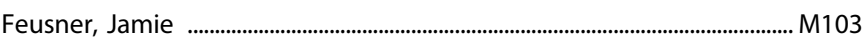

Fielding, Amanda ......................................................................................................W61

Fiellin, David .................................................................................................................... M167

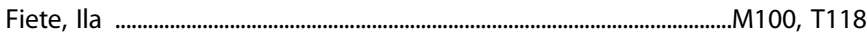

Fietze, Ingo ...................................................................................................M137, W150

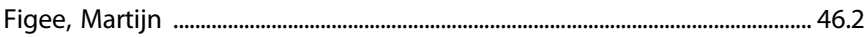

Figueroa, Jose ....................................................................................................................

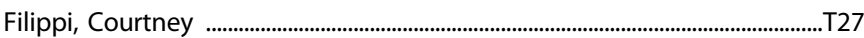

Filippov, Gleb …………………………………………………………......M139, T157

Fillinger, Clementine .....................................................................................................T38

Findling, Robert L. .................................................................................W35, W50

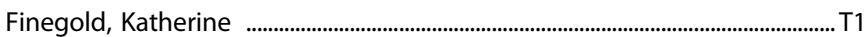

Fink-Jensen, Anders .................................................................................................W151

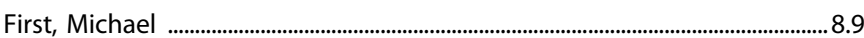

Fischer, Adina ...............................................................................................................W67

Fischer, Bernard ................................................................................................................ 21

Fischer, Corinne ....................................................................................................... W107

Fish, Kenneth ............................................................................................................... T132

Fisher, Hayley .................................................................................................................. M135

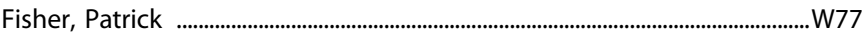

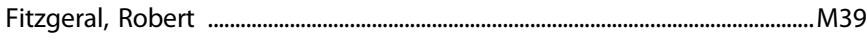

Fitzgerald, Paul .....................................................................................................................T92

Flagel, Shelly

27, 44, M187

Flanigan, Meghan

28.2, M192, W196

Fleig, Carline

T186

Fleischhacker, W Wolfgang

...T134

Fleming, Scott

Flint, Alastair

... W128

Flook, Elizabeth M116, T55

Flores, Cecilia
Flores, Christopher

Floresco, Stan

$\mathrm{T} 178, \mathrm{~T} 191$

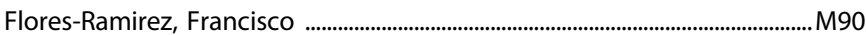

Floriou Servou, Amalia ................................................................................................. W8

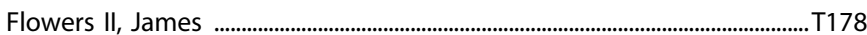

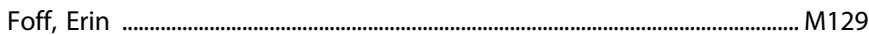

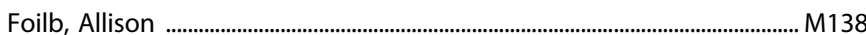

Folorunso, Oluwarotimi ...............................................................................M49, M118

Fontaine, Martine ................................................................................................... T128

Fonzo, Gregory ............................................................................................................. T5

Foran, William .......................................................................................................... M128

Forbes, Erika ............................................................................................33, M153

Ford, Judith .................................................................................... M130, T34, W136

Forde, Natalie J. .....................................................................................21, W128

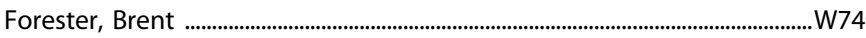

Forman, Mark ................................................................................................................ M129

Forte, Jennifer ............................................................................................................ T129

Fossati, Philippe ................................................................................................................T29

Fossler, Michael ..............................................................................................T125, W105

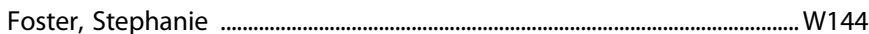

Fourgeaud, Lawrence ..................................................................................................... M88

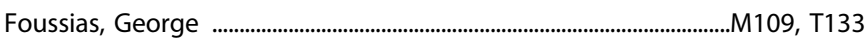

Fowler, Christie .............................................................................................. M181, M183

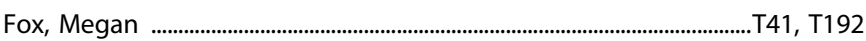

Fraiser, Claire M. ..................................................................................W112, W168

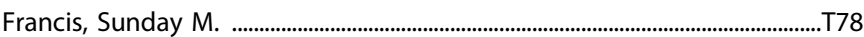

Franco, Daniela ........................................................................................................... T192

Frangou, Sophia ..................................................................................................... T198

Frank, Guido ..................................................................................................................T76

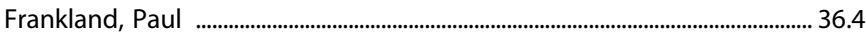

Frankovich, Kyle ...................................................................................................... T138

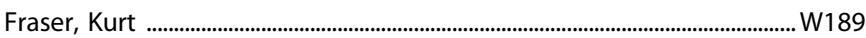

Frau, Daniela Virginia .....................................................................................................

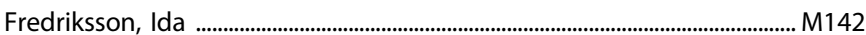

Free, R. Benjamin ............................................................................................................. T49

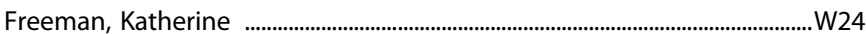

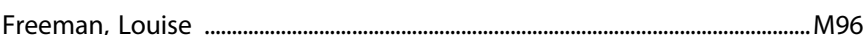

Freeman, Marlene ...........................................................................................T111, W59

Freitag, Gabrielle ........................................................................................................T27

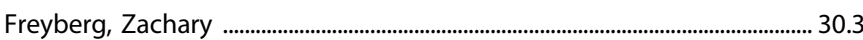

Fridman, Moshe ............................................................................................T109, W91

Fried, Jamie ...................................................................................................................... T44

Friedman, Allyson ............................................................................................................M51

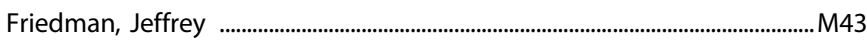

Friedmann, Peter ................................................................................................................. 50

Friend, Samantha ........................................................................................................T72

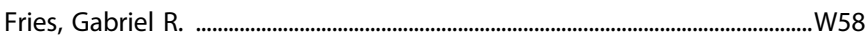

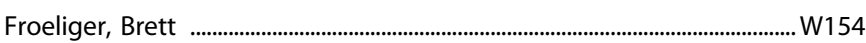

Froger-Colleaux, Christelle .................................................................................. T149

Fu, Dong-Jing ....................................................................................... T93, T95, W62

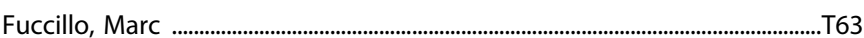

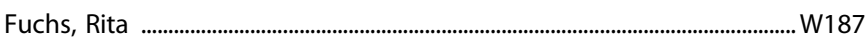

Fuertig, René ........................................................................................................ T94, W65

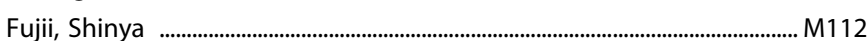

Fujita, Masayo ...............................................................................................M98, M127

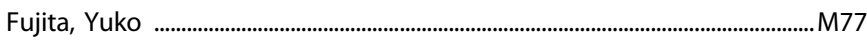




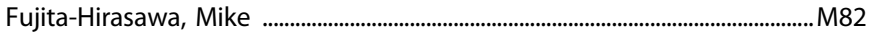

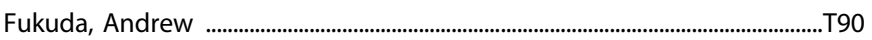

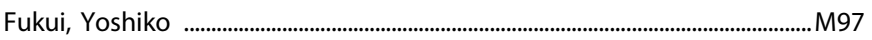

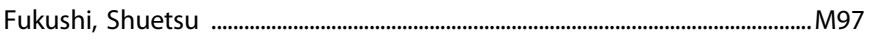

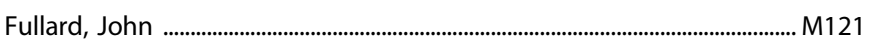

Fung, Lawrence ............................................................................................................W39

Funkhouser, Carter ........................................................................................................ M158

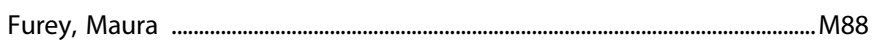

Furuyashiki, Tomoyuki ............................................................................................... M76

Gabbay, Vilma ........................................................................................................ T180

Gaccione, Peter ..............................................................................................................T111

Gagnon, Leona .................................................................................................... W163

Gajipara, Nileshkumar .......................................................................................T151

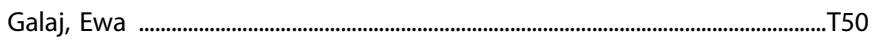

Gallego, Juan ................................................................................M113, T104, W127

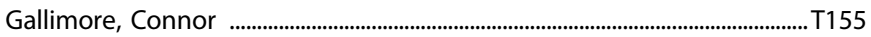

Galliou, Justine ...........................................................................................................W18

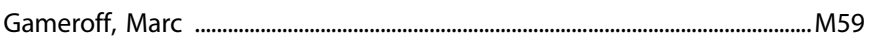

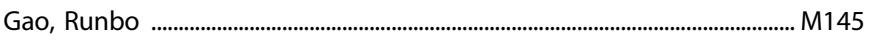

Gao, Shichao .............................................................................................................W104

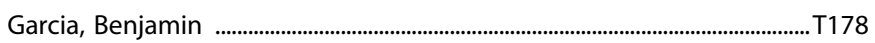

Garcia, Ronald ........................................................................................................... 3.4

Garcia de la Garza, Angel .....................................................................................T59

Garcia-Keller, Constanza ......................................................... M3, M150, W14, W183

Gardier, Alain .................................................................................................................T29

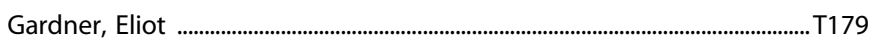

Garg, Sahil .................................................................................................................W120

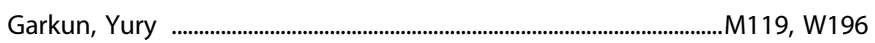

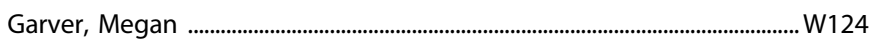

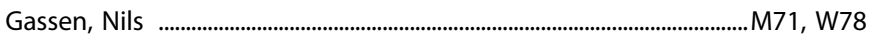

Gastinger, Matthew .......................................................................................... T163

Gatta, Eleonora ...............................................................................................................W79

Gawrysiak, Michael ................................................................................................ M164

Gee, Dylan $38.1,38.2, \mathrm{M} 24$

Gehrman, Philip .............................................................................................................T97

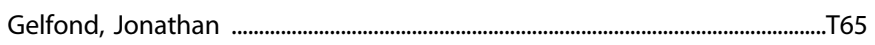

Gelpi-Dominguez, Ursula .......................................................................................W169

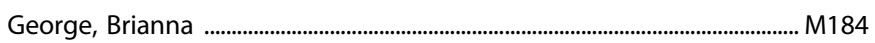

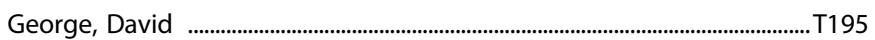

George, Mark ..................................................................................................................T199

George, Olivier ............................................................................... 7.4, M39, M168, M170

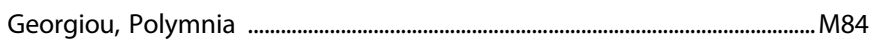

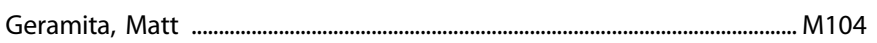

Gereau, Graydon ............................................................................................ M171, W48

Gerena, Jennifer ................................................................................................ M177

Gergues, Mark ..................................................................................................................T74

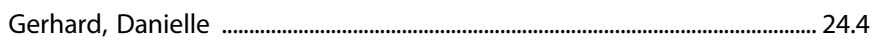

Germain, Pierre-Luc ........................................................................................................ W8

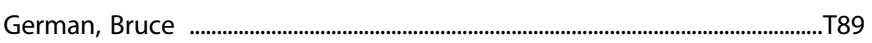

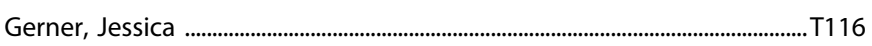

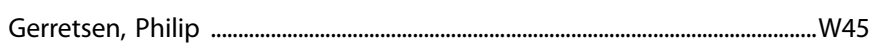

Gershon, Elliot .............................................................................................................. W198

Geuze, Elbert .................................................................................................................M9

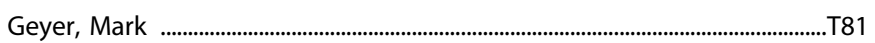

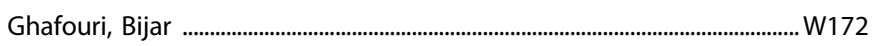

Ghoshal, Soham ...................................................................................................T38

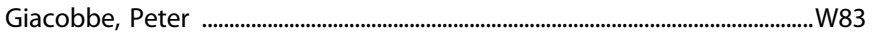

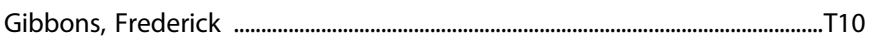

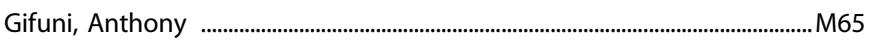

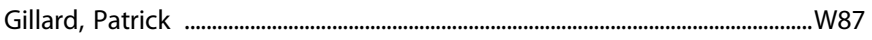

Gilman, Jodi ..................................................................................................................T182

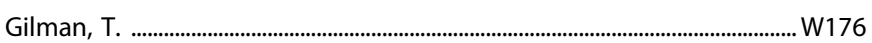

Gilmore, Casey ..........................................................................................................W106

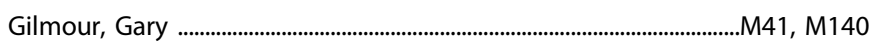

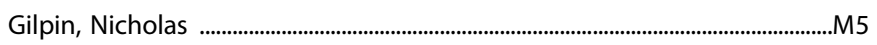

Giovanetti, Simone .................................................................................................. M141

Gipson, Cassandra ................................................................................................. M166

Girdhar, Kiran ............................................................................................................. M121

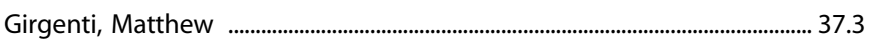

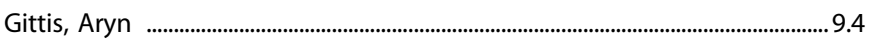

Giusepponi, Maria Elena .................................................................................W150

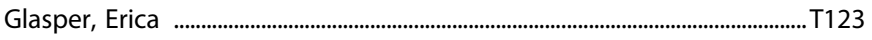

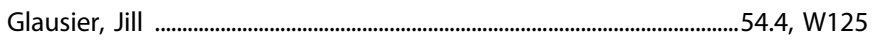

Glenn, Daniel ................................................................................................................T72

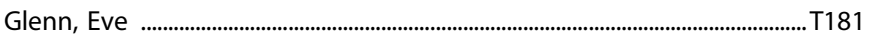

Glick, Ira .........................................................................................................T139, W44

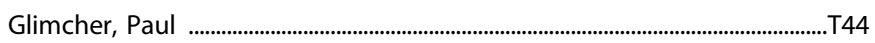

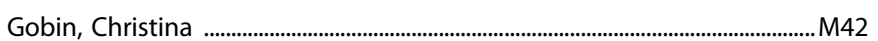

Godino, Arthur ..................................................................................................T18, W167

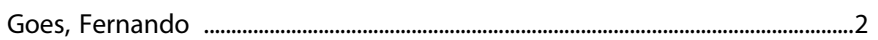

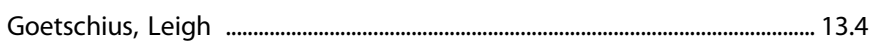

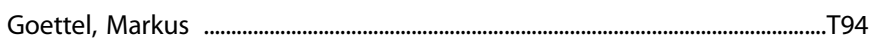

Goez Mogollon, Lina ................................................................................................ T111

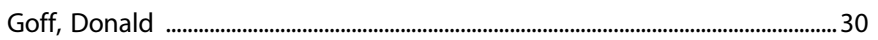

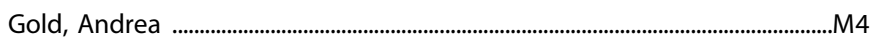

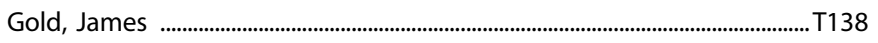

Goldberg, Andrew ..........................................................................................W122

Goldberg, Pablo H. ..............................................................................................T128

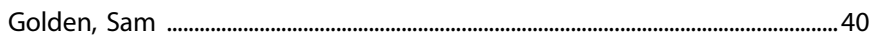

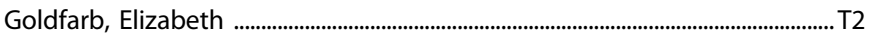

Goldman, Danielle ....................................................................................................... M60

Goldman, David ..................................................................................T83, T172, T195

Goldschmied, Jennifer ............................................................................................T97

Goldsmith, David ........................................................................................ T146, W129

Goldstein, Benjamin .................................................................................................... 12.4

Goldstein, Jill ........................................................................................................37.5, T96

Goldstein, Rita ..............................................................................................M154, M156

Goldwaser, Eric ...........................................................................................M46, M131

Gollub, Randy ..............................................................................................................T15

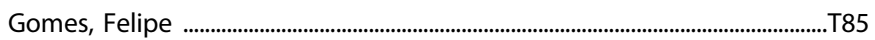

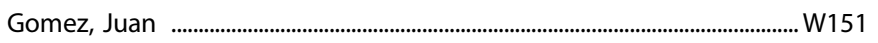

Gonçalves, Vanessa ............................................................................................W126

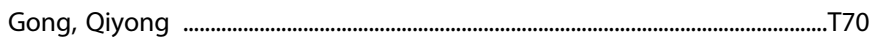

Gonsalves, Meghan ...................................................................................................T186

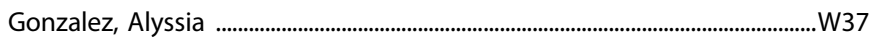

Goodkind, Madeleine .............................................................................................. T5

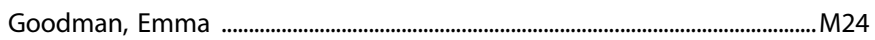

Goodrich, Lisa ...............................................................................................................T91

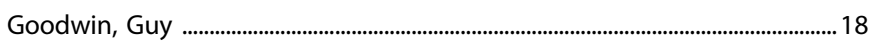

Gordon, Joshua .....................................................................................................21, T30

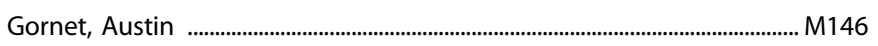

Goswamee, Priyodarshan .......................................................................................M94 
Gothelf, Doron

Gotlib, lan

...M65

Goto, Yukiori

Gould, Robert

M182

Gould, Todd

Goulet, Joseph

Grace, Anthony

Gracias, Alexa

Graff-Guerrero, Ariel

M155, T85

Grafman, Jordan

M112, W45, W107

Graham, Christine

....W75

Granata, Lauren

M111

Grant, Steven

..W11

Grant, Yanabel

......22

Grant-Beurmann, Silvia

....T47

Grasser, Lana

W168

Grassie, Hannah

...W17

Graves, Morgan

..W36

Gray, Madelyn

Grayson, Dennis

M149

Grazioplene, Rachael

...T129

Greally, John

.... W142

Greden, John

...W74

Green, Michael

4.5, T145

Green, ReJoyce

M152, W181

Green, Tamar

...T13

Greenberg, David

M197

Greene, Rachel K.

..M96

Greenstein, Dede

T116

Gregg, Allison

..M78

Gregory, Michael

T35, W32

Gregory-Flores, Adriana

M167, T179

Grennan, Laura

.T197

Griffiths, Roland

Grigorova, Yoana

Grillon, Christian

Grimm, Simone

...M75

Grodin, Erica

M105, W65

Groeschl, Martina

T42, W181

Grogan, Shayna

..T135

W187

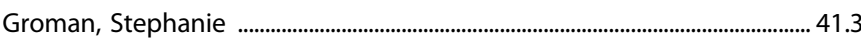

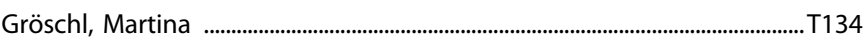

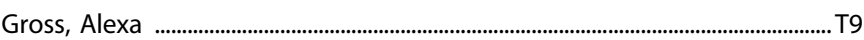

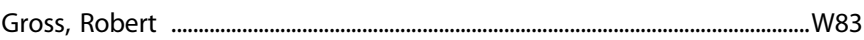

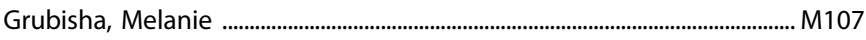

Grzenda, Adrienne ...............................................................................................W71

Gu, Shi .................................................................................................................

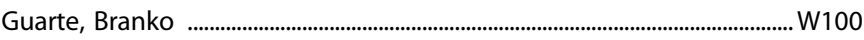

Guasoni, Paolo ..............................................................................................................W102

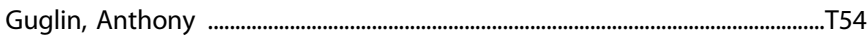

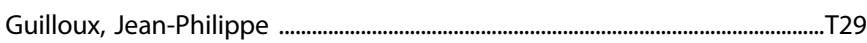

Gunawan, Tommy ..................................................................................................... T195

Gunduz-Bruce, Handan ..............................................................................T109, W91

Guo, Shien .................................................................................................................... T93

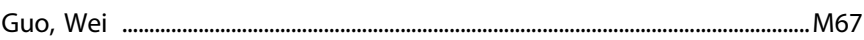

Guo, Weirui

.T175
Gur, Raquel

8.3, M197, T59

Gur, Ruben

M197, T59

Gur, Tamar

29.2

Gurney, Mark ............................................................................................................... W104

Gurrell, lan .........................................................................................................................W177

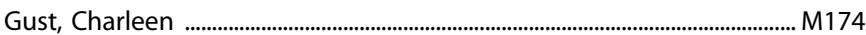

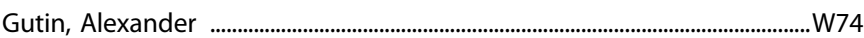

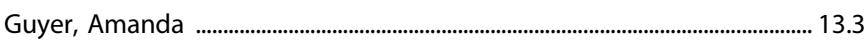

Gyertyán, István .................................................................................................................T113

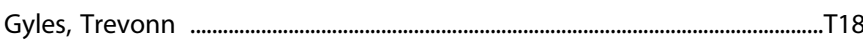

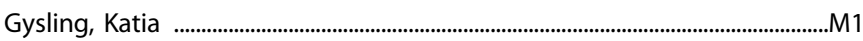

Ha, Sangtae ................................................................................................................W4

Haas, Shalaila .............................................................................................................. W120

Haass-Koffler, Carolina ................................................................................T185, T186

Haber, Suzanne .......................................................................................................... T130

Haberman, Jason .............................................................................................................

Hablitz, John .................................................................................................................... M133

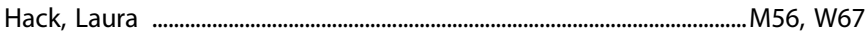

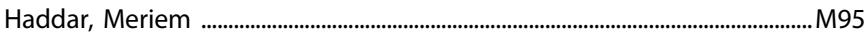

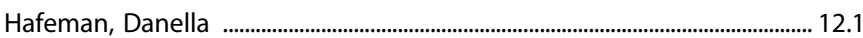

Hafenbreidel, Madalyn ....................................................................................... M143

Hafizi, Sina ..........................................................................................................W42

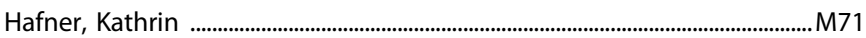

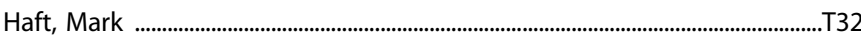

Hagihara, Hideo .................................................................................................... M200

Hagino, Yoko .......................................................................................M28, M98, M127

Hahn, Margaret ............................................................................30.1, 30.4, T37, W45

Hain, Daniel ..............................................................................................................W74

Hake, Sanjay ................................................................................................M126, T134

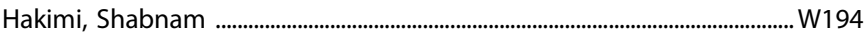

Halaris, Angelos ............................................................................................................. T24

Halberstadt, Adam ....................................................................................................... W200

Haley, Evan .......................................................................................................................... W4

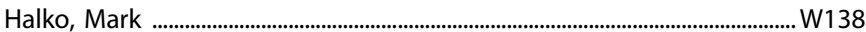

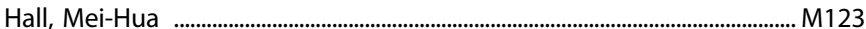

Hall, Nancy ......................................................................................................M139, T157

Haller, Simone ................................................................................................ T21, T22

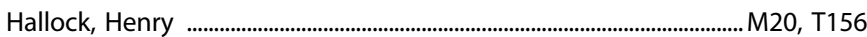

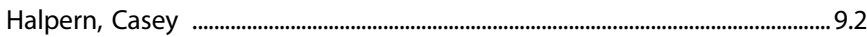

Hamani, Clement ...........................................................................................................W8

Hamborg, Madeline .................................................................................................W32

Hamilton, Holly K. .......................................................................................T34, W119

Hamm, Jordan ......................................................................................................... T155

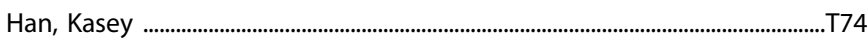

Han, Ming-Hu ...................................................................................................... M186, T98

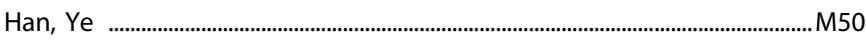

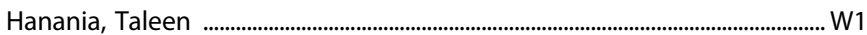

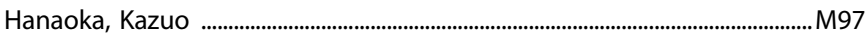

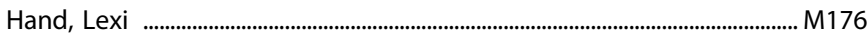

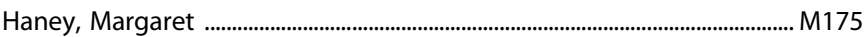

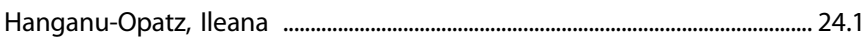

Hanham, Kate ...............................................................................................

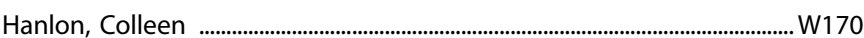

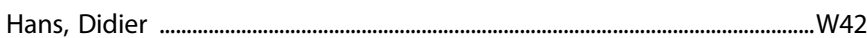

Hänsel, Katrin ...........................................................................................................W121

Hansen, Hanne ............................................................................................................W16 


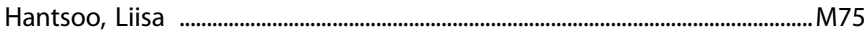

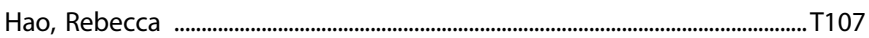

Hardiman, Gary ........................................................................................................ M172

Harkin, Andrew ..................................................................................................................992

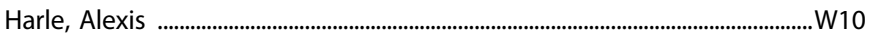

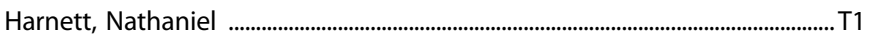

Haroon, Ebrahim ................................................................................................................. 15.3

Harper, Jennifer ................................................................................................................ T6

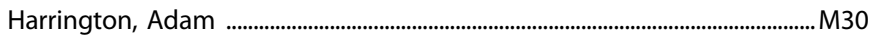

Harrington, Amanda .......................................................................................................W8

Harris, Alexander ..........................................................................................................T30

Harris, Erin ................................................................................................................W89

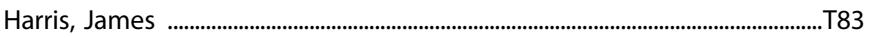

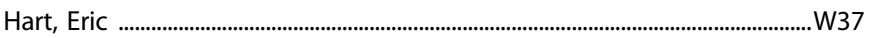

Hartmann, Jakob ........................................................................................................W78

Harvey, Deon .............................................................................................................T179

Harvey, Meredith .................................................................................................... T5

Harvey, Philip ........................................................................21, M114, T140, W132

Harvey, Theresa ................................................................................................ M1

Hasegawa, Junko ................................................................................................................

Hashimoto, Kenji ................................................................................................M77, T115

Hassan, Omron ............................................................................................................ W200

Haubrick, Kayla .................................................................................................................M36

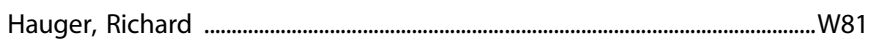

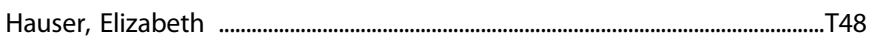

Hauser, Sheketha ......................................................................................................... M178

Haussmann, Sebastien ................................................................................................ M100

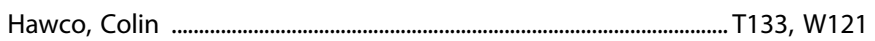

Hayashida, Masakazu ..................................................................................................99

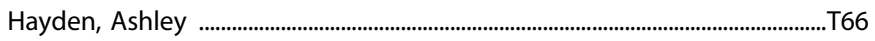

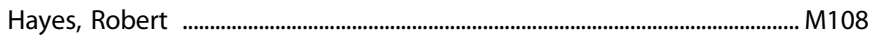

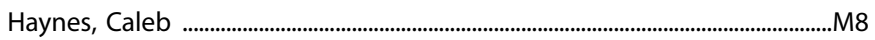

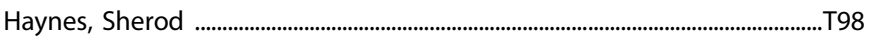

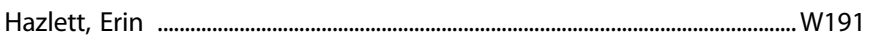

Heal, David ..................................................................................................T149, W51

Healy, Margaret .............................................................................................................W164

Heckers, Stephan ..................................................M115, M116, M117, T154, W133

Heilig, Markus ......................................................................................................... W172

Heinsbroek, Jasper ....................................................................................W143, W183

Hellemann, Gerhard ..............................................................................................T145

Heller, Carina ........................................................................................................W137

Heller, Elizabeth ............................................................................................................W7

Helmer, Cassandra ...................................................................................................... M107

Hen, Rene ......................................................................................................................T29

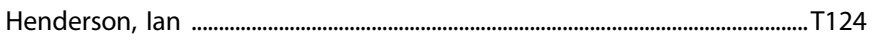

Hendrickson, Timothy .......................................................................................W106

Henningfield, Jack .................................................................................................W160

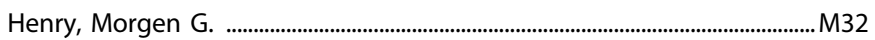

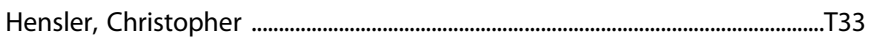

Herbstman, Julie .............................................................................................................T77

Heredia, Vanessa ......................................................................................................... T131

Herich, Lena ...................................................................................................................W65

Herman, James ..............................................................................................................W56

Herman, Kimberly ....................................................................................................W151

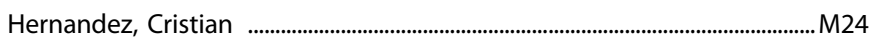

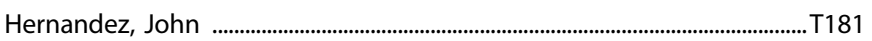

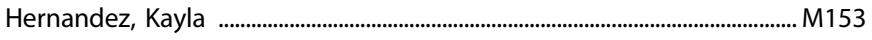

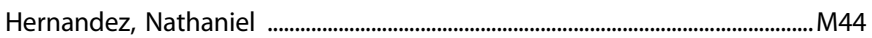

Hernández-Díaz, Sonia ...........................................................................................T111

Hernandez-Vasquez, Adriana ................................................................................. M183

Herrera, Shaynna ..................................................................................................W120

Herrera Charpentier, Andre ............................................................................. M177

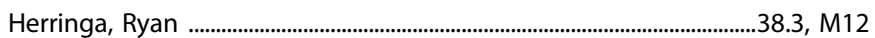

Herrmann, Evan ...................................................................................................... M175

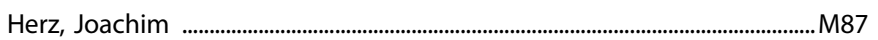

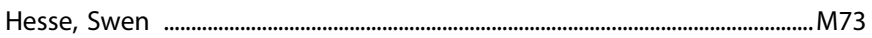

Hesselgrave, Natalie ......................................................................................................W99

Hetherington, Hoby …………………………………………………………………..... M128

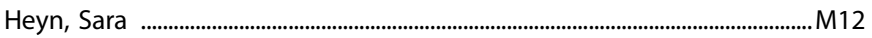

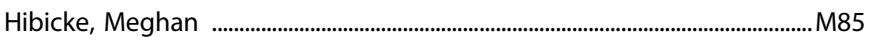

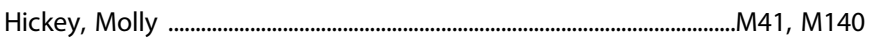

Hickey, Sarah .......................................................................................................... T182

Hickie, lan .................................................................................................................... M199

Hicks, Stephanie .........................................................................................................W10 W

Higgins, Amy ............................................................................................................ M123

Higley, J. Dee .............................................................................................................

Hikida, Takatoshi ............................................................................................................. M201

Hildebrand, Mariah ..................................................................................................... W125

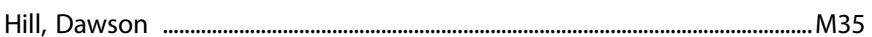

Hillmer, Ansel ....................................................M165, M191, T7, T45, W165, W171

Hindley, Lauren .........................................................................................................T90

Hiramoto, Takeshi ..........................................................................................................T81

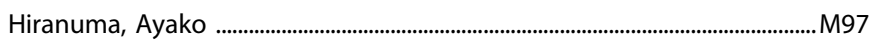

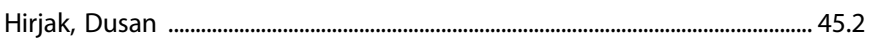

Hiroi, Noboru .............................................................................................................T81

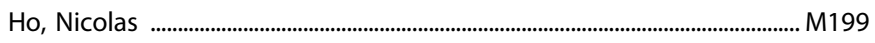

Ho, S. Shaun ............................................................................................. T167, W158

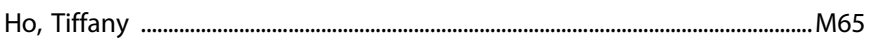

Hobbs, Taylor ...........................................................................................................T53

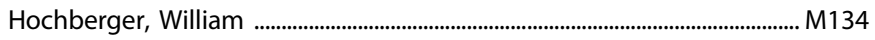

Hodebourg, Ritchy ....................................................................................................W14

Hoderbourg R, Ritchy ..................................................................................................

Hodes, Georgia ................................................................................................................. 3.1

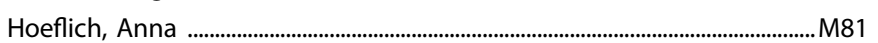

Hoeppner, Daniel ...............................................................................................................T81

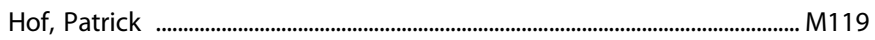

Hoffer, Lauren ......................................................................................................................M26

Hoffman, Ellen ........................................................................................................................

Hoffman, Gabriel ........................................................................................................ M121

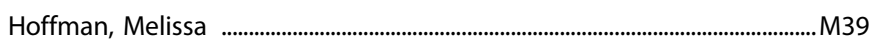

Hofford, Rebecca ......................................................................................M185, W167

Hogeveen, Jeremy ...........................................................................................W192

Hollander, Eric ...................................................................................................W24, W101

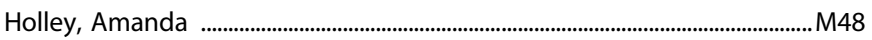

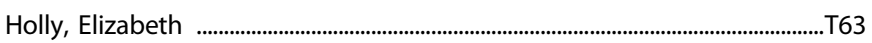

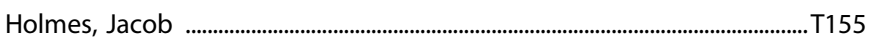

Holsen, Laura .................................................................................................... T15, T96

Holt, Emma ........................................................................................................T30

Holter, Kimberly ............................................................................................................ M182

Holt-Gosselin, Bailey ...................................................................................W67, W195

Holt-Lunstad, Julianne .......................................................................................................... 4.6

Holtzheimer, Paul .............................................................................................................W W 
Holze, Friederike

Homanics, Gregg

Hon, Olivia

Honda, Shiori

Honeycutt, Jennifer

Hong, Elliot

Hong, Sa-lk M46, M70, M131

Hood, Lauren E. W152

Hooker, Jacob M. .....T194

Hope, Bruce M63, M96

Hopf, Frederic

M142

Hopkins, Jordan

...T188

Hopkins, Seth

....M3

Hopkinson, Casey M54, M108, W96

Hopkinson, Craig

....W31

Hoptman, Matthew

M111

Horan, Bill

W128

Horga, Guillermo

.... M126

Horneffer, Yvonne

W122, W153

Horovitz, David

T195

Hossein, Shabnam

Hou, Wei

W193

Hougland, James

T179

Howe, James

.......T9

Howe, William

W148

Howes, Oliver

43.2

$\mathrm{Hu}, \mathrm{Che}$-Lin

....T124

$\mathrm{Hu}$, Xiuying

...T100

$\mathrm{Hu}$, Yang

..W47

Huang, Anna

Huang, Henry

M115

Huang, Jingyu

M165, T7, W165

Huang, Mei

Huang, Ming-Yi

....T101

.... W2

Huang, Songqiao

M80, T109, W91, W92

Huang, Xiaoqi

M126, T134

Huang, Yanhua

Huang, Yiyun

Huber, Kimberly

Huber, Rebekah

Huckins, Laura

Huckstep, Kate

Hudgens-Haney, Matthew

Huege, Steven

Huertas-Pérez, Carlos

Huestis, Marilyn

Hughes, Brandon

Hughes, Dylan

Huguenard, John

Humburg, Bree

Hume, Stacey

Hunker, Avery

Huo, Jason

Huppert, Theodore

Hurd, Yasmin

Hurley, Shaun
Husain, Mustafa

M78, W83

Hutchison, Kent

M174

Hutten, Nadia

Huys, Quentin

Hwa, Lara M71, M120, W109

Hyde, Thomas $M$

M19, T12

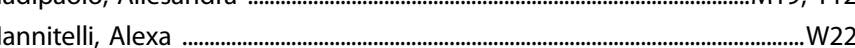

Ibanez-Tallon, Ines ................................................................................................W148

Ibarra, Christopher ...................................................................................................W18

Ibrahim, Hicham ........................................................................................................W68

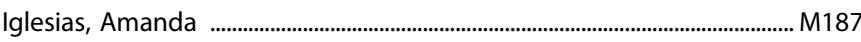

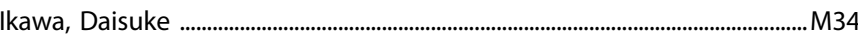

Ikeda, Kazutaka ..........................................................................28, M97, M98, M127

Ikehara, Minobu .......................................................................................................... M34

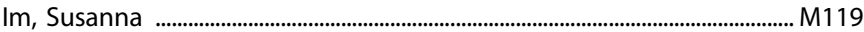

Imperatore, Julia ...........................................................................................................W170

Inamdar, Amir ...................................................................................................W177

Ing, Petr Kudlicka .....................................................................................................W137

Iñiguez, Sergio ....................................................................................................

Innis, Robert .......................................................................................................................... 15

Inslicht, Sabra ..............................................................................................W12, W16

Investigators, GENDEP ............................................................................................. T100

Investigators, T1000 ................................................................................................... T61

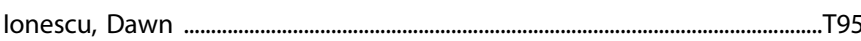

lorfino, Frank .......................................................................................................... M199

lqbal, Sidra .............................................................................................................. T106

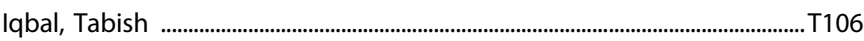

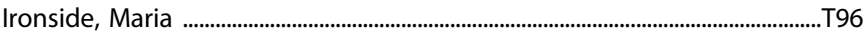

Iseki, Masako .................................................................................................................. M97

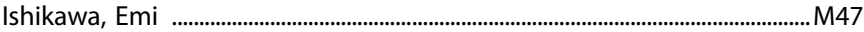

Ishima, Tamaki ....................................................................................................... M77

Ishizuka, Koko ....................................................................................................T32

Isiktas, Atagun ........................................................................................................... M190

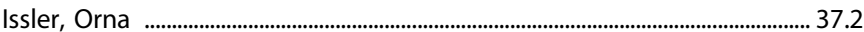

Itoga, Christy A. ..................................................................................................W28

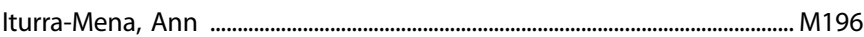

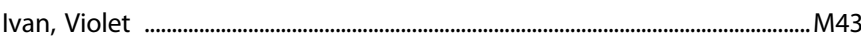

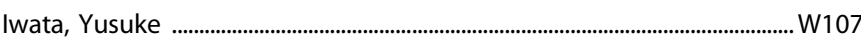

lyengar, Smriti ..........................................................................................................53, W1

Iyer, Anjali .......................................................................................................................... W10

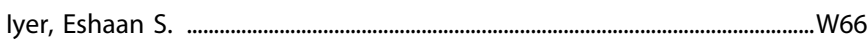

Izquierdo-Garcia, David ..........................................................................................96

Izuo, Naotaka .................................................................................................................. M95

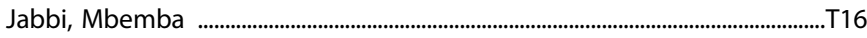

Jablonski, Michael .............................................................................................................W74

Jack, Mi'Anjel ....................................................................................................... T131

Jackson, Daphne .........................................................................................W164

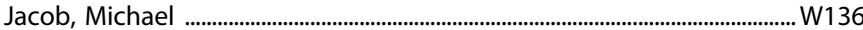

Jacob, Suma ………………………………………………………………....T78, W101

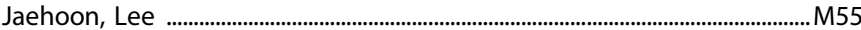

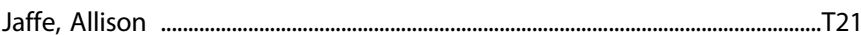

Jaffe, Andrew ..................................................................................... M71, T156, W109

Jaganjac, Adna .............................................................................................................W64

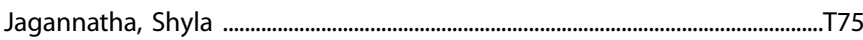


Jagannathan, Kanchana

M164, W155

Jahan, Aava B

....M63

Jain, Rakesh

James, Andrew

W164

James, Hyde M102

Jamie, Jamie D

Jamieson, Carol ....T95

Janak, Patricia

W189

Janes, Amy

W138

Jani, Martin

W137

Jank, Alexander

M73

Jaquish, Abigail

M170

Jarcho, Johanna

.....M8

Jaric, Ivana

W142

Jasarevic, Eldin

51.4

Javitt, Daniel

.......6

Jaworowicz, David

M129

Jaworska, Natalia

M13

Jaymes, Asa

W179

Jenck, Francois

...W15

Jenkins, Bryan

W135

Jenkins, Lisanne

.M26

Jentsch, James

......22

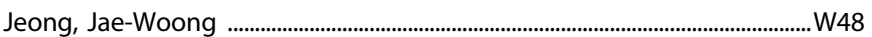

Jessica Deslauriers, Jessica

.....M2

Jeste, Dilip

4, T152, W130

Jeste, Shafali

$26,26.2$

Jha, Manish

5.5, T68, W57, W59, W199

Ji, Gang

....W47

Ji, Jie Lisa

... 47.3

Jiang, Huabei

...T101

Jiang, Shixie

... T101

Jiang, Shui

..T100

Jiang, Ying

Jimenez Chavez, C. Leonardo

T175

Jimmy, Jagan

...T56

Jin, David

M31

Jin, Hua

M22, T137

Jin, Xin

20.5, T118

Jing, Deqiang

...T127

Jo, Booil .

...... T5

Jo, Young .................................................................................................................W159

Jo, Young Chan ..............................................................................................................M33

Joanlanne, Andrea ...................................................................................................T104

Joëls, Marian

...W78

Joffe, Max

$.48 .1,48.3$

Johannesen, Jason K

W119

Johansen, Sys S.

John, Majnu

W77

Johnson, Amy

...W121, W127

Johnson, David

M16

...T124

...T184

Johnson, Michael

W148

Johnson, Paul

M75

Johnson, Rachel

T174

Johnson, William

...T65
Johnston, Camille ………………………………………………………………….......M8

Jones, Carrie K. ............................................................................................................. M182

Jones, Marie ...……………………………………………………………………... M174

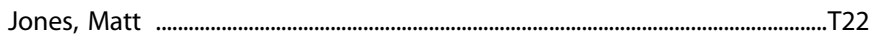

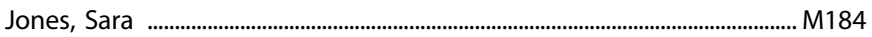

Jones, Tom ...................................................................................................................... M124

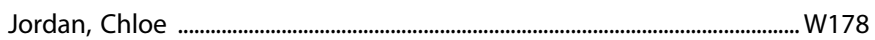

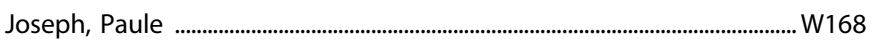

Joshi, Sonalee ........................................................................................................ M82, T8

Joshi, Yash ................................................................................................................ M134

Jou, Roger ...........................................................................................................W10

Jovanovic, Tanja ...........................................................14.4, M18, T1, T3, T82, W17

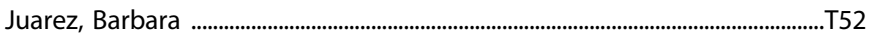

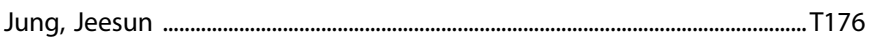

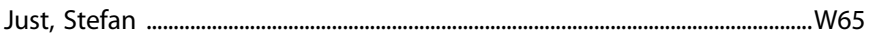

Justice, Amy ........................................................................................................... M167

K Namboodiri, Vijay Mohan ................................................................... M102, T53

Kable, Joseph ....................................................................................................W114

Kaczkurkin, Antonia ........................................................................................55, T59

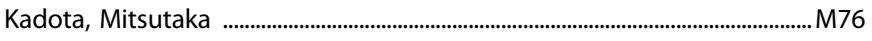

Kahn, Danielle .......................................................................................................................T15

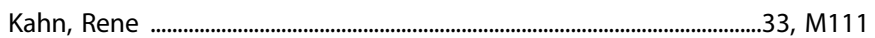

Kalivas, Peter ........................M3, M144, M150, M172, T166, W14, W143, W183

Kallioniemi, Elisa ....................................................................................................... T25

Kallupi, Marsida ...........................................................................................M39, M170

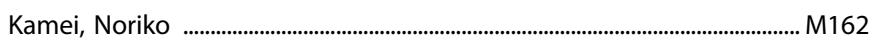

Kamillar-Britt, Philip ................................................................................................. T180

Kaminsky, Jesse ..............................................................................................................T19

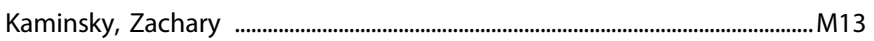

Kamis, Danielle ............................................................................................................ T139

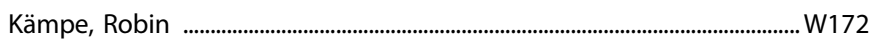

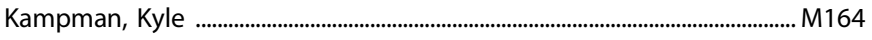

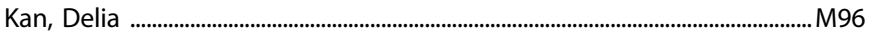

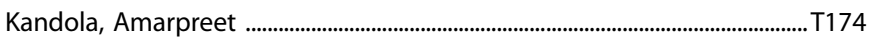

Kane, Maureen ...……………………………………………………………………... W112

Kanekar, Shami ................................................................................................................ 3.2

Kanes, Stephen J. ....................................................................M80, T109, W91, W92

Kang, Gina ................................................................................................................ T81

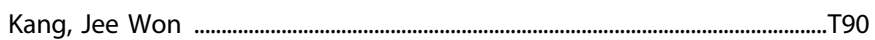

Kang, Seungwoo ………………………………………………………….....W41, W139

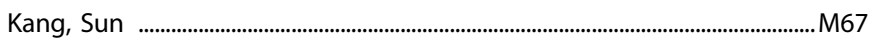

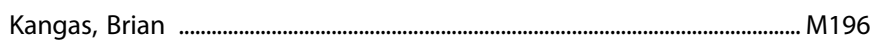

Kano, Shin-ichi .............................................................................................................T64

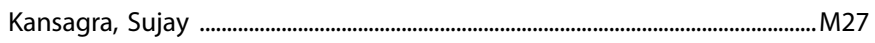

Kapinos, Michael ............................................................................................M165, T7

Karabinas, Isabella ...............................................................................................T19

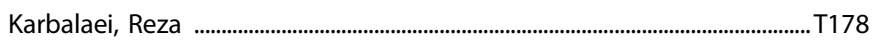

Kardon, Randy .....................................................................................................W10

Karkhanis, Anushree ..................................................................................................T189

Karne, Saumya ...………………………………………………………………... W144

Karoly, Hollis ......................................................................................................... M174

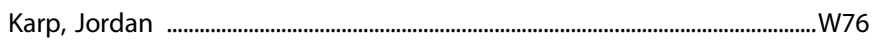

Karst, Edward ................................................................................................................W83

Kasai, Shinya ............................................................................................................M97

Kash, Thomas ..............................................................................................M192, W182

Kaskie, Rachel ........................................................................................................ M132 


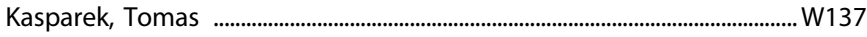

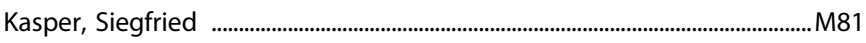

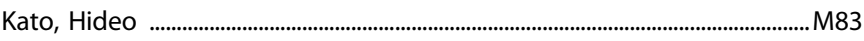

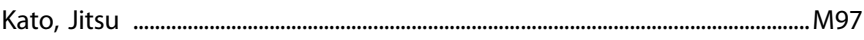

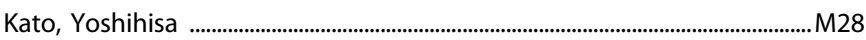

Kauer, Julie ................................................................................................................. 32.4

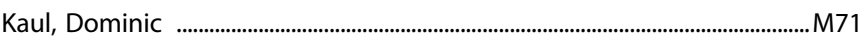

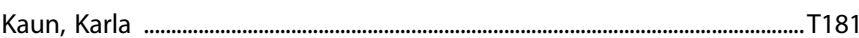

Kavalali, Ege .................................................................................................. M53, M87

Kavanagh, Sarah ........................................................................................................ M1 10

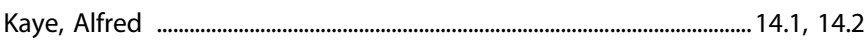

Kaye, Walter ................................................................................................................. T8

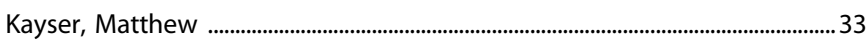

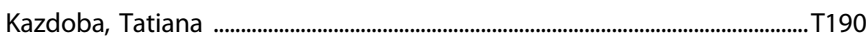

Kearns, Angela M ......................................................................................................M3

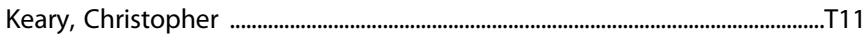

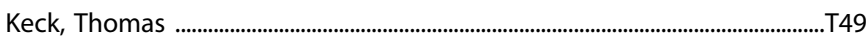

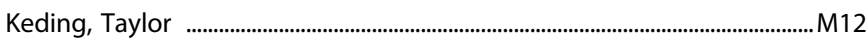

Keedy, Sarah .........................................................................................................W198

Keefe, Richard ............................................................................................M126, W118

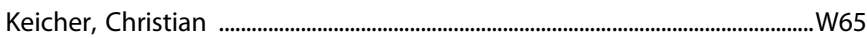

Kellendonk, Christoph ....................................................................................................... 24

Keller, Arielle S. .............................................................................................. M56, W195

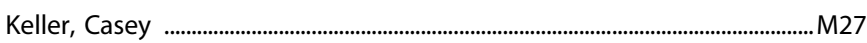

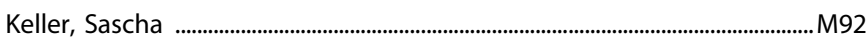

Kelliher, Julia C. .......................................................................................... T174, W161

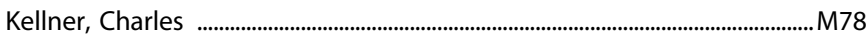

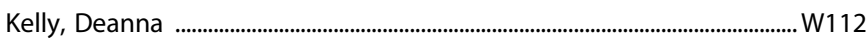

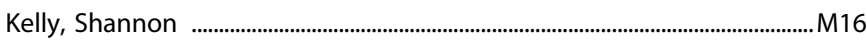

Kelmendi, Benjamin ........................................................................................... T129

Kelsall, Nora ............................................................................................................... M59

Kelsoe, John .............................................................................................W54, W94

Kendzior, Brian ........................................................................................................... T182

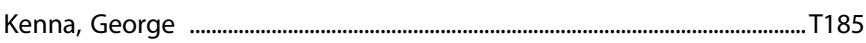

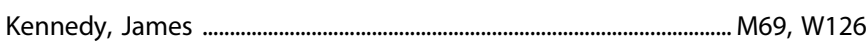

Kennedy, Sidney ......................................................................................................W83

Kenny, Paul ......................................................................36, 36.2, T38, W148, W177

Kent, Justine ....................................................................................................M54, M108

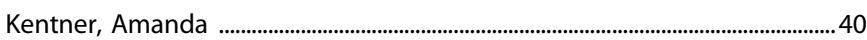

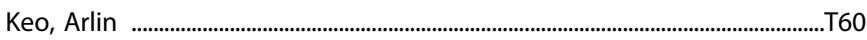

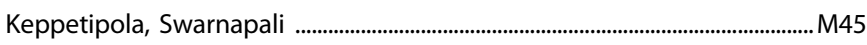

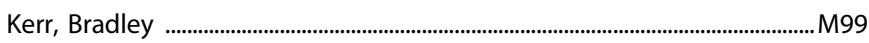

Keshavan, Matcheri .....................................................................................................W198

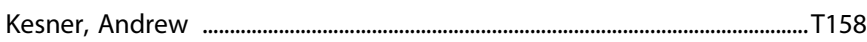

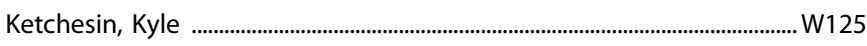

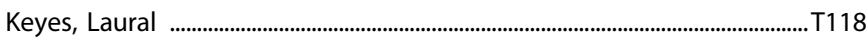

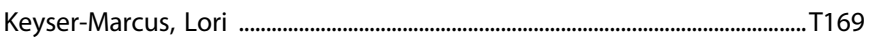

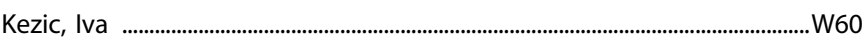

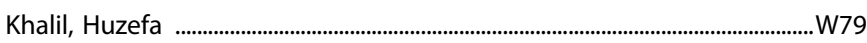

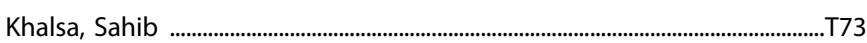

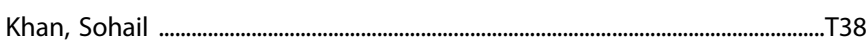

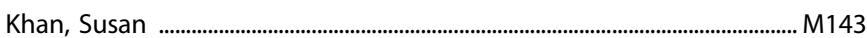

Kheirbek, Mazen ………………………………………………………………………... T74

Khokhar, Jibran ........................................................................................................... W135

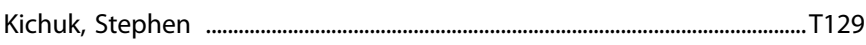

Kidambi, Neha .................................................................................................................... T152

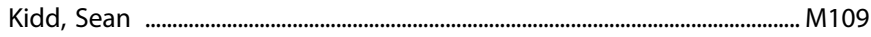

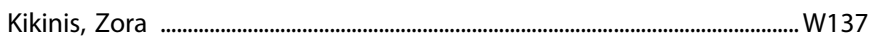

Kilts, Clinton ................................................................................................................. W164

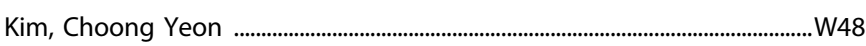

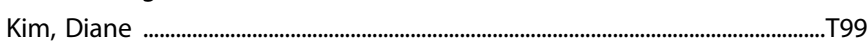

Kim, Jenny .................................................................................................................W4

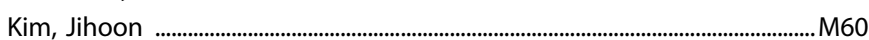

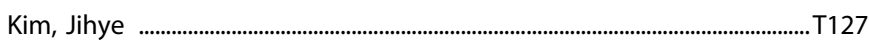

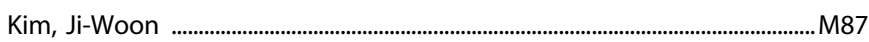

Kim, Jiyoon ................................................................................................................... M201

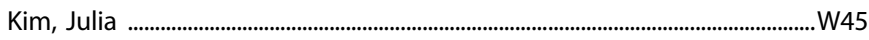

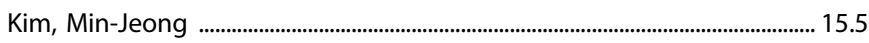

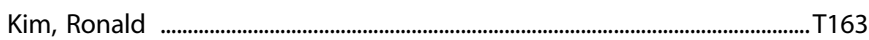

Kim, Sam-Moon ........................................................................................................ W163

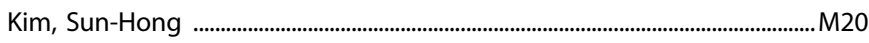

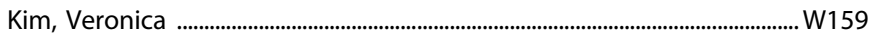

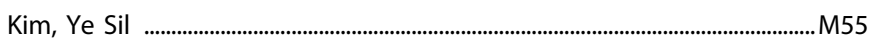

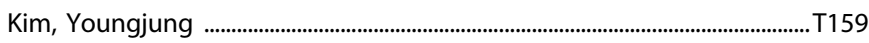

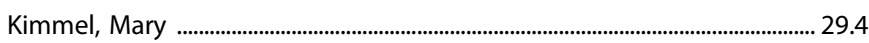

Kim-Schulze, Seunghee ........................................................................................... T180

Kinard, Jessica L. .........................................................................................................M96

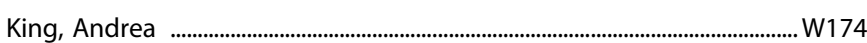

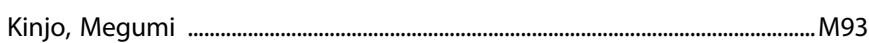

Kiraly, Drew ..................................................................................................M185, W167

Kircanski, Katharina ............................................................................... 5.3, T27, W36

Kirlic, Namik .......................................................................................M25, T61, W84

Kirshenbaum, Jaclyn ....................................................................................................

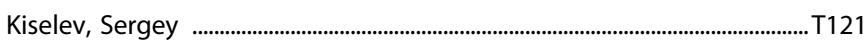

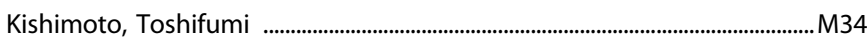

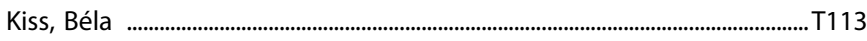

Kitamura, Takashi .....................................................................................................W131

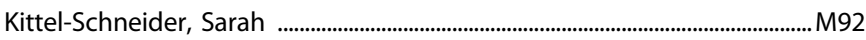

Klaus, Federica ............................................................................................................ T103

Klei, Lambertus ................................................................................................................ W124

Klein, Landon ............................................................................................................ W200

Kleinman, Joel ........................................................................................M71, M120, W109

Klengel, Claudia .........................................................................................................W78

Klengel, Torsten ................................................................................................M18, W78

Klimes-Dougan, Bonnie ............................................................................................... M35

Klodniki, Mike ............................................................................................................... M170

Klumpp, Heide ...................................................................................................... M158, T56

Knapp, Christopher ................................................................................................. T191

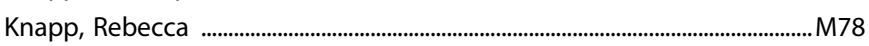

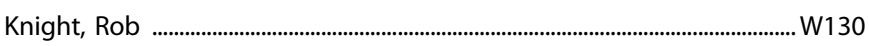

Knippenberg, Anna .......................................................................................................... M86

Knudsen, Gitte ...................................................................................................W77

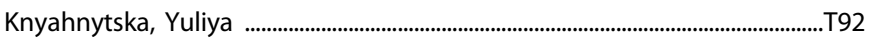

Ko, Insu ..........................................................................................................W54

Kobayashi, Kazuto ..........................................................................................M98, M127

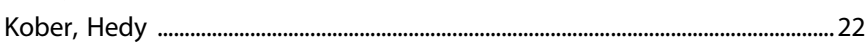

Koblan, Kenneth ..........................................................................21, M54, M108, W96

Kochunov, Peter .................................................................................M46, M70, M131

Kockmann, Tobias ............................................................................................................. W8

Koek, Wouter .................................................................................................................. M147

Koenigsberg, Harold .............................................................................................W191

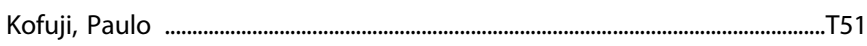


Kohn, Philip

W32

Koike, Hiroyuki

M119, W196

Koistinaho, Jari

T142

Koizumi, Teruki

M83

Kolassa, Iris-Tatjana

Kolivakis, Theodore

M194

Kollins, Scott

W83, W186

Komori, Takashi

Kong, Mi-Seon

M34

Konova, Anna

Koob, George

Kopelman, Jared

Korah, Hannah

Kortagere, Sandhya

Korte, Jeffrey

Kosciolek, Tomasz

Koshiyama, Daisuke

Koskuvi, Marja

Kotajima, Hiroko

Kotnana, Bhuvaneswari

Kotov, Roman

Kotz, Juliana

Koukouli, Fani

Koustova, Elena

Kozauer, Susan

Kozel, Frank

Kozloff, Nicole

Kraguljac, Nina

Kraines, Morganne

Kramar, Eni

Kramer, Edgar

Kramer, Michael

Kraus, Christoph

Kreisl, William

Kremens, Rebecca

Kretsge, Lisa

Kribakaran, Sahana

Krimmel, Samuel

Krishnan, Harish

Krishnan-Sarin, Suchitra

Kroll, Danielle

Kronman, Hope

Krueger, Angeline

Krueger, Bob

Kruyer, Anna

Krystal, John

Kubera, Katharina

Kubicki, Marek

Kuhn, Brittany

Kuhn, Cynthia

Kuiper, Lindsey

Kulikova, Alexandr

Kulkarni, Praveen

Kumar, Anand

Kumar, Sanjeev

Kummerfeld, Erich

44, T44, W153
Kundakovic, Marija

W142

Kupfer, David

18,33

Kuplicki, Rayus

T61, T73

Kuraku, Shigehiro

.M76

Kushner, Steven

M29

Kutlu, Munir

.M16, M190

Kuypers, Kim .............................................................................................W61

Kvarta, Mark

M70, M131

Kwako, Laura

T172, T195

Kwok, Claudina

.... T188

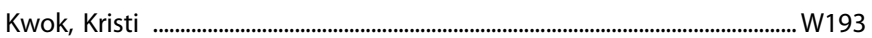

L. Barrigon, Maria ...........................................................................................................W43

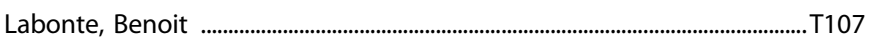

Lacy, Ryan ............................................................................................................... M184

Laezza, Fernanda .......................................................................................................... M49

LaGoy, Alice ............................................................................................................. M132

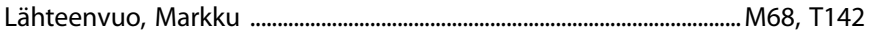

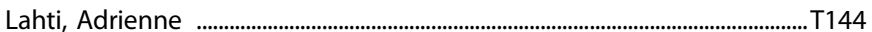

Lai, Meng-Chuan .............................................................................................. M21, T197

Lallai, Valeria ............................................................................................................... M181

Laloudakis, Yiani .........................................................................................................T150

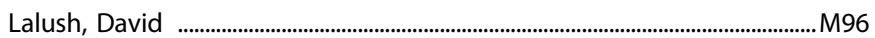

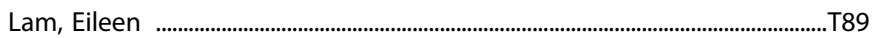

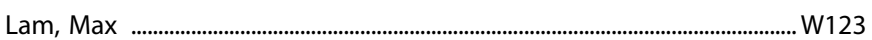

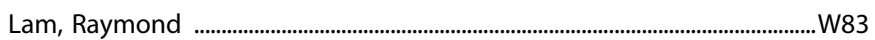

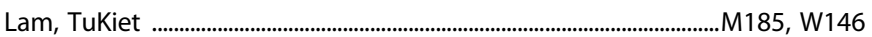

Lam, Vicky .......................................................................................................................W39

Laman-Maharg, Abigail ............................................................................................. T107

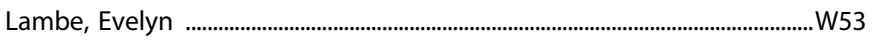

Landa, Yulia .................................................................................................................. 8.8

Landry, Ishani ..........................................................................................139, T157

Lane, Rosanne ............................................................................................................T95

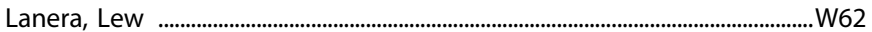

Lang, Ariel .....................................................................................................................W81

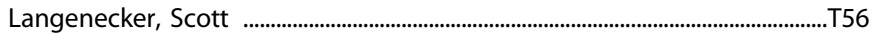

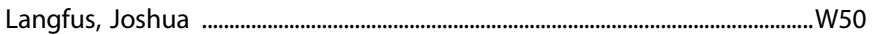

Langleben, Daniel D. ..........................................................................................W15

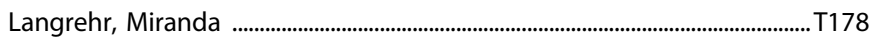

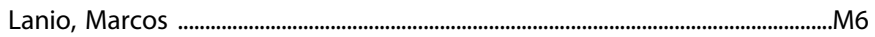

Lanzenberger, Rupert ................................................................................................... M81

Lao-Peregrín, Cristina .............................................................................................T127

Lapidus, Rachel .........................................................................................................T73

Lardenoije, Roy ..............................................................................................M18, W78

Lasser, Robert .................................................................................................T109, W91

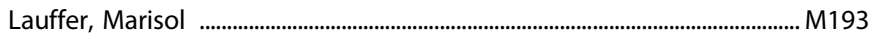

Laurence, Michael .....................................................................................................T139

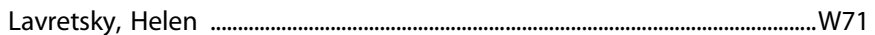

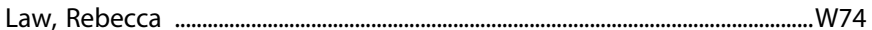

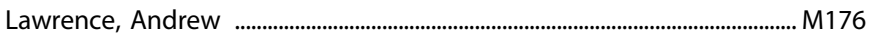

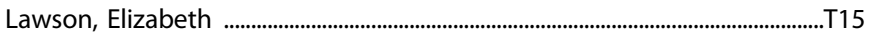

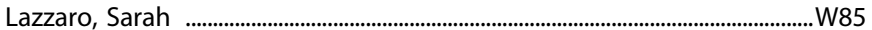

Le, Quan-son ...............................................................................................................W21

Le, Thang ...................................................................................................................... T173

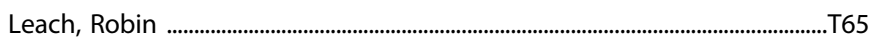

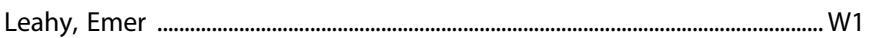

Leal Santos, Sofia .........................................................................................................M6 
LeBois, Lauren

Lecourt, Hugues

Lecza, Bernadette

...W15

Lee, Adam

M60

Lee, Amy

Lee, Chris

Lee, Ellen

Lee, Francis

Lee, Heemin

Lee, Jenna

Lee, Jisoo

Lee, John

Lee, Junghee

Lee, Mary

Lee, Phil

Lee, Young-A

Lee, Zo-Ann

Leger, Damien

Legget, Kristina

Leggio, Lorenzo

Legius, Eric

Lehmann, Vanessa

Lehtonen, Sarka

Lei, Kelly

Leibenluft, Ellen

Leipold, David

Leiser, Steven

Lekander, Alex

Lemieux, MacKenzie

Lencz, Todd

Lenroot, Rhoshel

Lenze, Eric

Leonard, Carly

Leonardo, Eduardo

Leow, Alex

LeRoux, Melissa

Lesko, Emma

Leslie, William

Lesscher, Heidi

Letavic, Michael

Letherby, Bethany

Leutgeb, Stefan

Levin, Frances

Levine, Kayla

Levis, Sophia

Levitt, James

Lewis, Cathryn

Lewis, Charles

Lewis, Crystal

Lewis, David

Lewis, Martin

Leyrer-Jackson, Jonna M.

Leyton, Marco

Lezak, Kim

Li, Anfei .

Li, Chiang-shan

M166, T194

... $\mathrm{M} 138$

...T127

....T173
$\mathrm{Li}$, Chunbo

Li, Guanya

$\mathrm{Li}$, Haihong

$\mathrm{Li}$, Hao

$\mathrm{Li}$, Jim

Li, Qingqin

$\mathrm{Li}$, Shuwen

Li, Songye

Li, Xiaobai

Li, Yan

Li, Yuli

Li, Zibo

Liang, Ana

Liao, Vicky

Liberzon, Israel

Libster, Avi

Licinio, Julio

Lieberman, Abby

Liechti, Matthias

Light, Gregory

Lijffijt, Marijn

Lim, Byungkook

Lim, Hyoyoung

Lim, Kelvin

Lim, Keunpoong

Lima, Camila Nayane de Carvalho

Lin, Alexander

Lin, Da-Ting

Lin, Han-Yu

Lin, Hongyu

Lin, Li

Lin, yingxi

Lindenmeyer, Hannah

Lindley, Steven

Lindsley, Craig W.

Ling, George

Ling, Ruth

Linnet, Kristian

Lintz, Tania

Lisanby, Sarah

Liss, Michael

Liston, Conor

Liu, Chunyu

Liu, Fang

Liu, I-Yuan

Liu, Keith

Liu, Rui ..

Liu, Shelley

Liu, Xin-An

Lix, Lisa

Lizano, Paulo

Lobo, Mary Kay

Locke, Adam

Lockhart, Sedona

Lockwood, Jonathan
Locher, Rebekka

... 17

...W47

.M80, W92

..T118

...M69

W103

...W75

W165

W168

M108

.T140

... M96

M119, W196

....T67

... 82

.T118

.T105

W190 ...W61

M134, M198, W100, W119

M55, T106

... 52.2

...W48

...22, M130, T78, W106

W171

...W58

..T67

52.3

..W8

M135

M143

52.1

W191

.... T5

M182

W198

W195

...W77

W159

11, 19, M78

....T65

.T19

.T141

W112

.T136

...W59

..T80

...W43

W148

...W42

W198 
Lodge, Daniel

Loebel, Antony

M136, W134

Lofaro, Olivia

M54, W96

...T196

Logan, Ryan

49.1, 49.5, W163

Lohoff, Falk T176

Lombroso, Sonia

Lönnqvist, Jouko

Lopez, Alberto

Lopez, Joelle

Lopez, Marcelo

Lopez, Maria

Lopez, Sarah

Lopez-Guzman, Silvia

Lord, Brian

Loren, Alexandra

Losak, Jan

Lossie, Amy

Lotfipour, Shahrdad

.... W7

T142

M16, T165

W66

W180

W191

M119, W196

..22, T44

M88, M89

..W98

W137

25.1

M179

Lotter, Tobias

M105

Lourenço, Joana

T126

Love, Tiffany

27.3

Lovenberg, Timothy

M88, M89

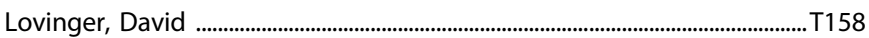

Lowes, Daniel ...............................................................................................................T30

Lozano, Andres ....................................................................................................................W8

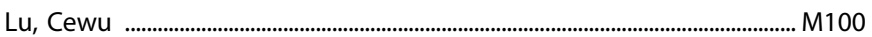

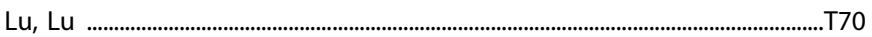

Luc, Oanh ........................................................................................................... M196

Luck, Steven ..................................................................................................................... T138

Lugo-Candelas, Claudia .....................................................................................T102

Luis, Angelica ..................................................................................................................W54

Luna, Beatriz ........................................................................................................ M128

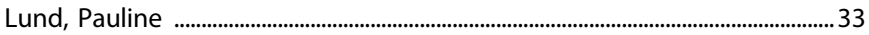

Lunerti, Veronica ................................................................................................... M172

Lunsford-Avery, Jessica ..............................................................................................M27

Luo, Sunny ..................................................................................................................... M100

Lustberg, Daniel ...........................................................................................................W22

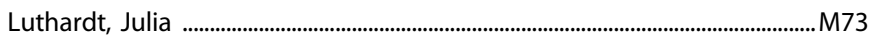

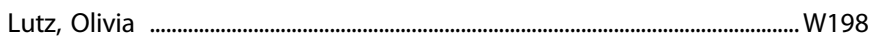

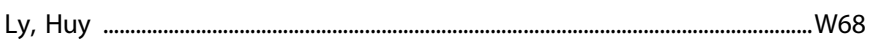

Lyall, Amanda .....................................................................................................................T15

Lydon-Staley, David ............................................................................................................ 22

Lyew, Thandi .................................................................................................................W76

Lynn, Peter …………………………………………………………………………….... W1 16

Lyon, Krissy .................................................................................................................T91

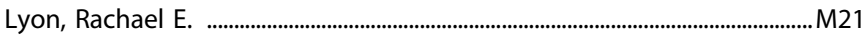

Lyu, Ilwoo ........................................................................................................... W133

Lyu, Jinpeng ……………………………………………………………………..... W104

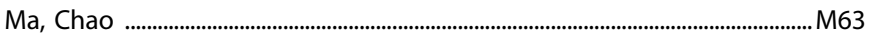

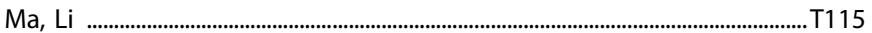

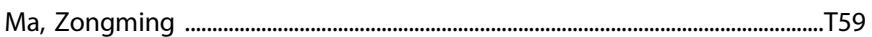

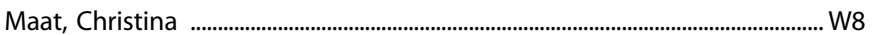

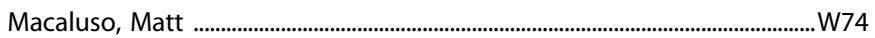

Maccario, Priscilla ...................................................................................................... W196

MacDonald, Matthew ...............................................................................................W124

MacDougall, Derek ...................................................................................................... W18
MacInnes, Jeffrey J.

W194

Mack, Salome Rebecca

T135, W65

MacNamara, Annmarie

.... T4

Macpherson, Tom

M201

Maddock, Richard

T143, T145

Madore, Michelle _.............................................................................W63

Madras, Bertha ................................................16

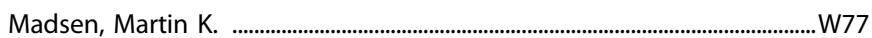

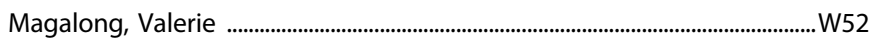

Maguire, Jamie ........................................................................................................................ 48.5

Mahfouz, Ahmed ..............................................................................................................T6

Mahler, Stephen ……………………………………………….......34.1, M162, T170

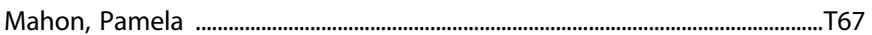

Maienschein-Cline, Mark ....................................................................................... M149

Maihofer, Adam ............................................................................................................T72

Majid, DS-Adnan ..........................................................................................................T87

Makinodan, Manabu ................................................................................................. M34

Makris, Nikos ...........................................................................................................W137

Malacon, Karen ..................................................................................................W159

Malaker, Pias ............................................................................................M154, M156

Malaktaris, Anne .................................................................................................W81

Malhotra, Anil ..........................................................M58, M113, T104, W121, W127

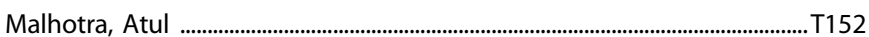

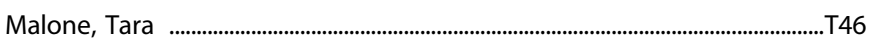

Manca, Elias ....................................................................................................................... M38

Manca, Letizia ............................................................................................................... M181

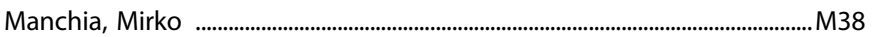

Mancino, Michael ...................................................................................................W164

Mandeville, Joseph .........................................................................................................W16

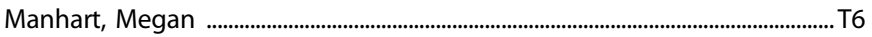

Mani, Bharath .............................................................................................................T179

Manning, Elizabeth .......................................................................................... M102, M104

Mano, Marsel ............................................................................................... T135

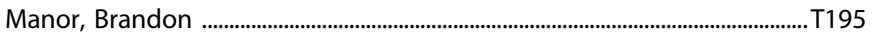

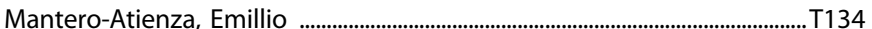

Manvich, Daniel ......................................................................................................T187

Manza, Peter ..................................................................M159, W151, W156, W173

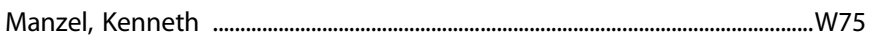

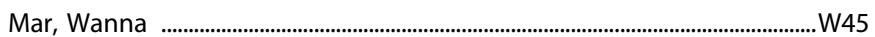

Marchant, Roman .............................................................................................. M199

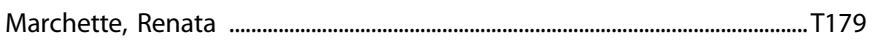

Marder, Stephen ............................................................................................................T145

Margolis, Amy ............................................................................................................T77

Margolis, Russell ........................................................................................................ M114

Maria Rios, Cristina .............................................................................................. W184

Marin, Marie-France ………………………………………………………………..... T3

Marks, William ..........................................................................................................W131

Marlin, Bianca Jones ........................................................................................................ M15

Marrero-Cristobal, Gigliola ....................................................................................W169

Marrocco, Jordan ......................................................................................................W79

Marsh, Rachel ...............................................................................................T31, T128

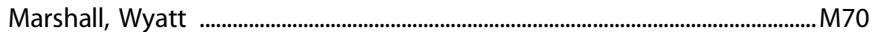

Marstrand-Joergensen, Maja ...............................................................................W77

Martens, Kristina ...................................................................................................... T100

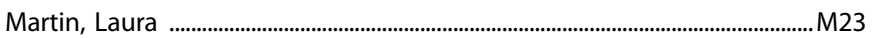

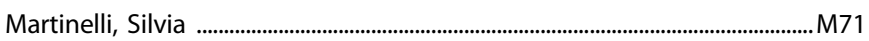


Martinez, Dalia

Martinez, Maria

T124

Martinez-Rivera, Freddyson

T130

Martínez-Rivera, Freddyson J.

Martinowich, Keri

....T18

Marton, Tobias

M20, T156, W109

Marusak, Hilary

Marwari, Subhi

Marx, Wolfgang

T105

Masand, Prakash

Masi, Paul

Mason, Barbara

Mason, Brittany

Mason, Natasha

Massa, Nicholas ...W61

Mastrodonato, Alessia

W119, W129

Mateo, Yolanda

Mates, Sharon

Mathalon, Daniel

M6, T114

.T158

21, T110, T148

Matheos, Dina 6, M130, T34, W95, W119, W136

Mathew, Sanjay J.

...T165

Mathews, Maju

M55, T106

Mathieu-Frasier, Lauren ....T93

Mathur, Brian

....T11

Matoba, Ken

T62, W190

Matosin, Natalie

. M71

Matsumoto, Mitsuyuki

....T81

Matsushita, Karin

M112

Matsushita, Kazutoshi

....M76

Matta, Jose

M89

Matthews, Gillian

Matuskey, David

.... M100

May, Jensen

M165, T7, T45, W171

Mayberg, Helen

M135

Mayeli, Ahmad

...46, W83

Mayer, Grace

Mayfield, Dayne

Mayleben, David

Maynard, Kristen

Mayo, Leah

McBride, Molly

McCall, Adelina

McCall, Jordan

McCall, W. Vaughn

McCalley, Daniel

McCann, Katharine

McCarthy, Michael

McCauley, Sarah

McClain, Lora

McClernon, Francis

McClernon, Joseph

McClintock, Shawn

McCloskey, Michael

McClung, Colleen

McColl, Alison

McCracken, James
McCullough, Kenneth

M138, W78

McCullumsmith, Robert

...W56

McDermott, Tim

..W84

McDonald, Jeffery

McDonald, Kelsey

McDonnell, David M111

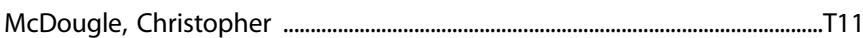

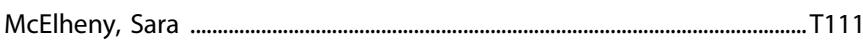

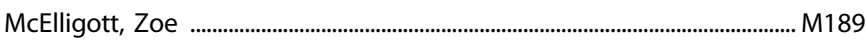

McElroy, Susan ..............................................................................................................W87

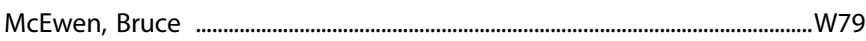

McGinn, Adrienne .................................................................................................. M167

McGinty, Jacqueline ..............................................................................................W140

McGlade, Erin .................................................................................................T79, T122

McGlashan, Thomas H ............................................................................................W119

McGowan, Josephine ...............................................................................................W19

McGuinness, Amelia ....................................................................................................M64

McHugo, Maureen ............................................................. M116, M117, T154, W133

McIntyre, Roger ..............................................................................................T148, W87

McKee, Sherry ................................................................................................................W165

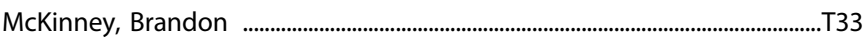

McKinney, Walker ......................................................................................................

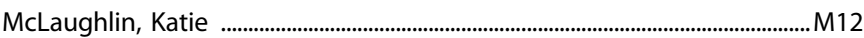

McLean, Caitlin ................................................................................................................ M135

McLean, Samuel ............................................................................................................W17

McLoughlin, Declan M. .............................................................................................M92

McMahon, Francis ........................................................................................ 2, M70, T16

McMullen, David .......................................................................................................... 19.1

McNamara, Nora ...................................................................................................W101

McNamara, Robert ................................................................................................ W197

McPherson, Katherine .................................................. M159, W151, W156, W173

McQuaid, Robyn ........................................................................................................

McQuail, Joseph .............................................................................................................. W5

McQuiston, Adam .......................................................................................................... M94

McTeague, Lisa ...............................................................................................................T57

Meagher, Mary ................................................................................................................ T4

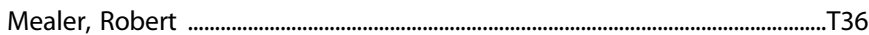

Meckel, Katherine .....................................................................................M185, W167

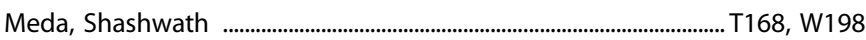

Medhurst, Laurie .................................................................................................M41, M140

Meijer, Mandy ........................................................................................................................T60

Meijer, Onno ...................................................................................................... T60, W78

Meinecke, Douglas ......................................................................................................... 54.1

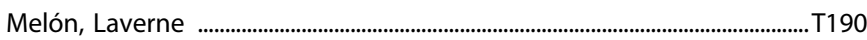

Meloni, Anna .................................................................................................................... M38

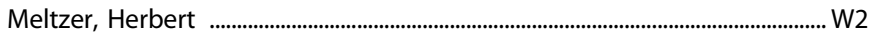

Meltzer-Brody, Samantha .................................................................29, M80, W92

Melugin, Patrick ...............................................................................................16, M190

Menard, Caroline ..................................................................................................36.1, 36.3

Mendez, Adriana ....................................................................................................T52

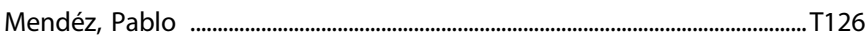

Mendez-David, Indira .............................................................................................. T29

Mennen, Anne ............................................................................................................W64

Mennenga, Sarah .......................................................................................................... T46

Mennes, Maarten .............................................................................................. M105, W65

Merikangas, Kathleen ................................................................................................. 


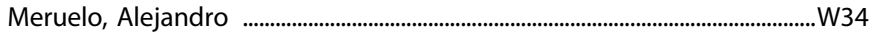

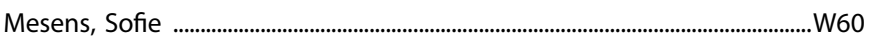

Messing, Robert ........................................................................................................ M141

Messinger, John ...............................................................................................................T44

Meyer, Heidi .......................................................................................................39.2, T14

Meyer, Jeffrey ...................................................................................................... 15.1, 15.2

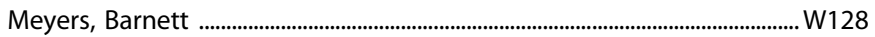

Michaelides, Michael ...............................................................................................W151

Michal, Matthias ........................................................................................................M92

Michopoulos, Vasiliki ....................................................................2. 23.1, 23.2, T3, W9

Mickey, Brian .......................................................................................................... 27.1

Middleton, Jason ..............................................................................................................

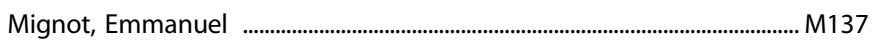

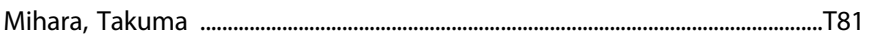

Mikati, Marwa ................................................................................................................. M171

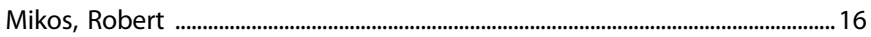

Milad, Mohammed ......................................................................................................... T3

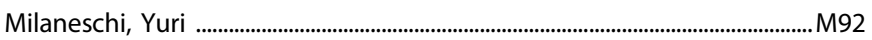

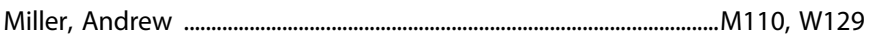

Miller, Brian .................................................................................................... T146, W129

Miller, Courtney ............................................................................................................ M143

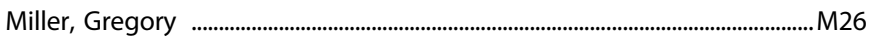

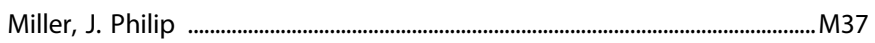

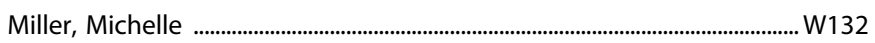

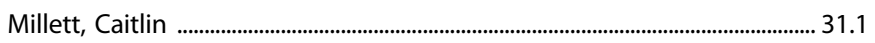

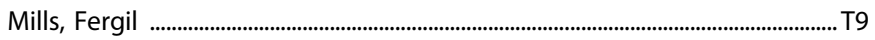

Mills, Jeffrey A. ............................................................................................................T70

Mimura, Masaru ....................................................................................M83, M93, M112

Minhajuddin, Abu ........................................................................................T68, W199

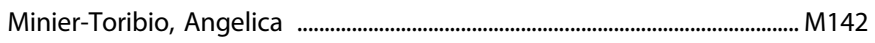

Misa, Madhusmita ....................................................................................................T15

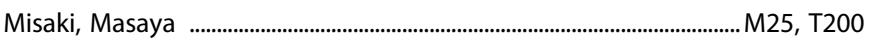

Mischel, Nicholas .............................................................................................M91, W8

Mishra, Himanshu .....................................................................................................W54

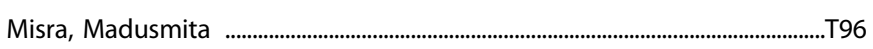

Missig, Galen ............................................................................................................... M138

Mitchell, Braxton ...........................................................................................................

Miura, Yuka .................................................................................................................... M51

Miyakawa, Tsuyoshi ................................................................................................. M200

Mizrahi, Romina .....................................................................................................43, 43.5

Modabbernia, Amirhossein ...................................................................................T198

Moeller, F. Gerard .......................................................................................... 11, T169

Moeller, Scott ............................................................................................................ T180

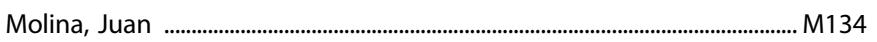

Moline, Margaret ………………………………………………………….......M139, T157

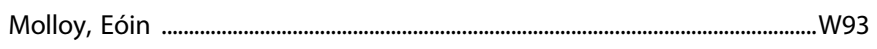

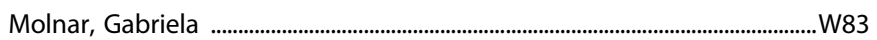

Molofsky, Anna Victoria ............................................................................................T74

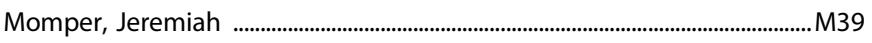

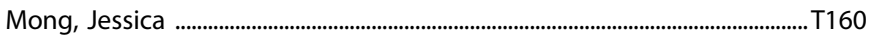

Monk, Catherine ....................................................................................22.1, 51.2, W27

Monk, Christopher ....................................................................................................... 13

Monroe, William ................................................................................................................. T144

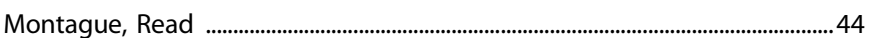

Montano, C. Brendan ..................................................................................W87, W90

Monteggia, Lisa ................................................................................................. M53, M87

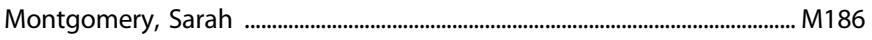

Montmerle, Martin ...........................................................................................................T126

Moody, Teena D. ...................................................................................................T8

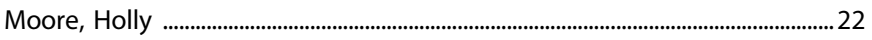

Moore, Raeanne ......................................................................................................W132

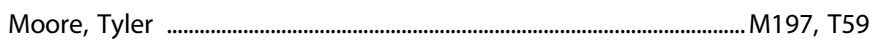

Moose, Jacob .............................................................................................................. T179

Morales-Silva, Roberto .............................................................................................W169

Moran, Lauren ............................................................................................................... W138

Moreira, Thais .............................................................................................................W90

Morel, Carole .............................................................................................................. M18

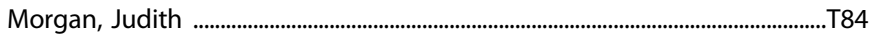

Morilak, David .............................................................................................M86, T65

Morishita, Hirofumi ........................................................................24.3, M119, W196

Morita, Tomonari ..................................................................................................M47

Moron-Concepcion, Jose ....................................................................................T54

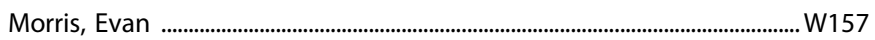

Morris, James .................................................................................................................. M163

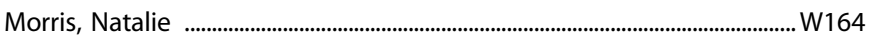

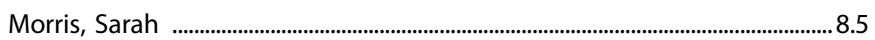

Morrison, Kathleen ............................................................................................................ 51.3

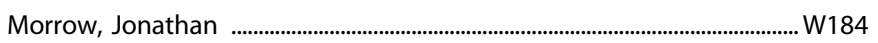

Morton, Mitchell .............................................................................................................T30

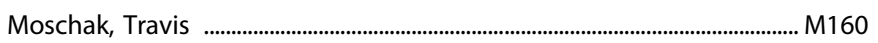

Mosconi, Matthew ...............................................................................................M23, T86

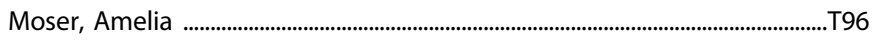

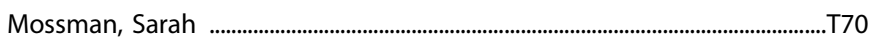

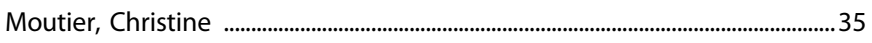

Moxon-Emre, Iska ............................................................................ M21, T197, W128

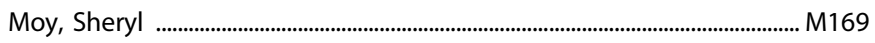

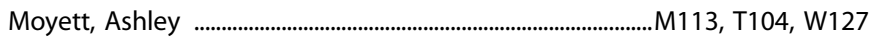

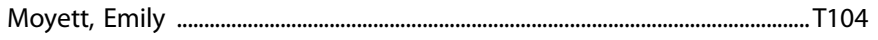

Mubeen, Muhammad ..........................................................................................T168

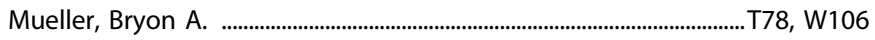

Mueller, Martina ..........................................................................................................M78

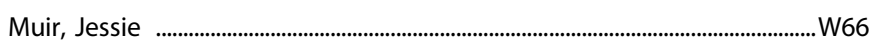

Mukamel, Eran .................................................................................................................... M195

Mullett, Jennifer ...........................................................................................................T11

Mullins, Teagan ......................................................................................................W192

Mulsant, Benoit ....................................................................... T92, T133, W107, W128

Mulvey, Bernard ......................................................................................................T23

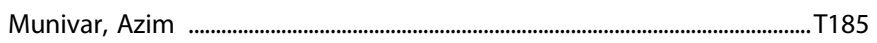

Muñoz Zamora, Andrea ...........................................................................................M6

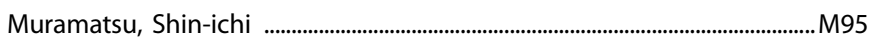

Murano, Tomoyuki ................................................................................................... M200

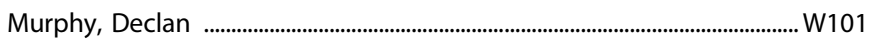

Murphy, Geoffrey ............................................................................................W184

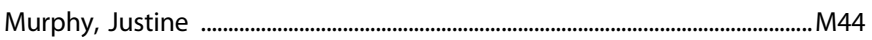

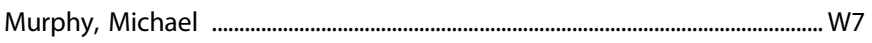

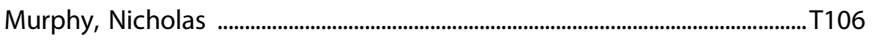

Murphy, Zackari ..........................................................................................W141, W18

Murray, Robin ..................................................................................................................... 8.2

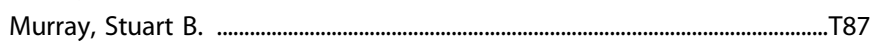

Murtagh, Lorainne ..................................................................................................W101

Murty, Vishnu .......................................................................................................T1, W17

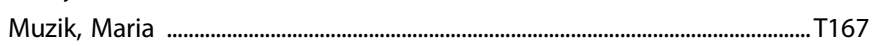


Myers, Bryn

Myers, Michael

M17, T6

Myin-Germeys, Inez

Nabeel, Nabulsi

Nabekura, Junichi

Nabel, Elisa

M119, W196

Nabulsi, Nabeel M165, T7, T45, W157, W171

Naganawa, Mika ...T171

Nagashima, Masako ....T81

Naim, Reut

T21, W36

Nairn, Angus

...W146

Najafzadeh, Soheila

T7, W165

Najibi, Seyed Morteza

M132

Nakagome, Kazuyuki

...T134

Nakahara, Tomomi

...M93

Nakajima, Shinichiro

M93, M112

Nakamura, Mitsuteru

Nakayama, Kyoko

Nakazawa, Kazutoshi

M133

Nall, Rusty

W183

Namba, Mark

Namburi, Praneeth ..................................................................................................... T118

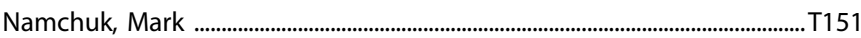

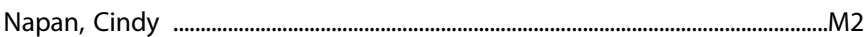

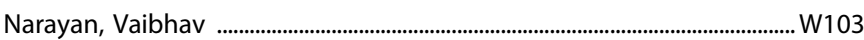

Nash, Abigail I. ............................................................................................... T93, W62

Nasiri, Nima ............................................................................................................ W119

Nasrallah, Henry ........................................................................................................ T136

Natividad, Luis ...................................................................................................................T39

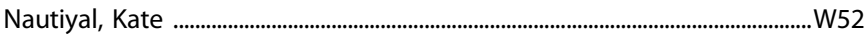

Navarra, Rachel ........................................................................................................ T191

Nawaz, Uzma ...........................................................................................................W138

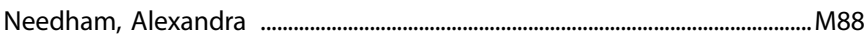

Neigh, Gretchen $23.3,35, \mathrm{M} 94$

Neisewander, Janet

$22, \mathrm{~T} 184$

Nelson, Lauren

....W87

Nestler, Eric

37, M148, M186, T18, T107

Neufeld, Nicholas

W128

Neuhofer, Daniela

M150

Neunuebel, Joshua

.. .40

New, Antonia

33, W191

Newcomer, John

M37

Newman, Amy

T49, W178

Neylan, Thomas W12

Ngai, John .................................................................................................................... 19.2

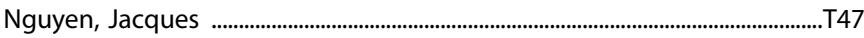

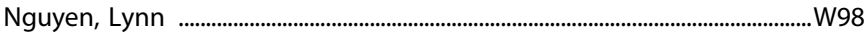

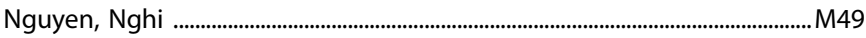

Nguyen, Nhien .......................................................................................................... T89

Nguyen, Tanya ....................................................................................................... W130

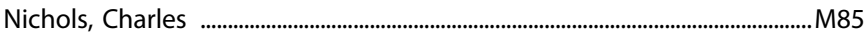

Niciu, Mark

Nickels, Stefanie M195, M196

Nickerson, Lisa M123

Nicol, Ginger

Nicole, Hinds
Nieddu, Mariella

... M38

Niedtfeld, Inga

M105, W65

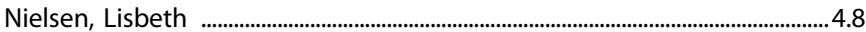

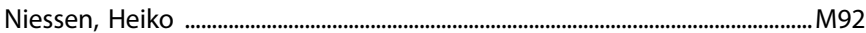

Nieto, Steven ..............................................................................................W181

Nievergelt, Caroline ...........................................................................................T72

Nikulin, Vadim .....................................................................................................W93

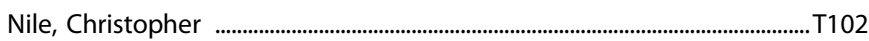

Nishi, Mayumi .............................................................................................................................

Nishizawa, Daisuke ...........................................................................................................

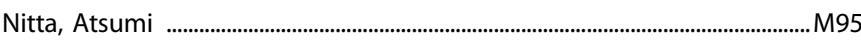

Niwa, Minae ..............................................................................................................W8

Niznikiewicz, Margaret A ....................................................................................W119

Njathi-Ori, Catherine ....................................................................................................... 35

Noall, Madison ............................................................................................................ M116

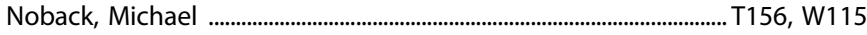

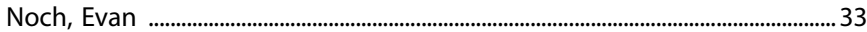

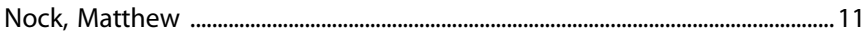

Noda, Yoshihiro ………………………………………………………........M93, M112

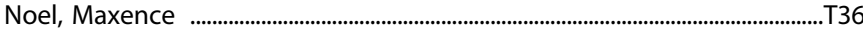

Noeldeke, Jana ............................................................................................W10

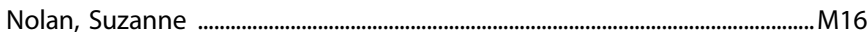

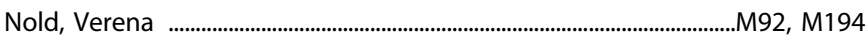

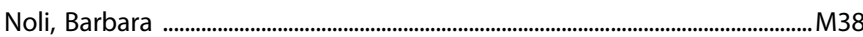

Noonan, James ........................................................................................................................ 20.4

Nooner, Kate ...................................................................................................................W34

Norbury, Agnes ..............................................................................................................W43

Norman, Kenneth .......................................................................................................W64

Norman, Kevin ..........................................................................................M119, W196

Norman, Luke ............................................................................................................W25

Norman, Nina ........................................................................................................... M182

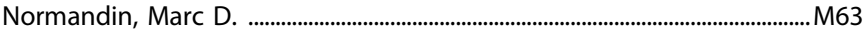

Norris, Haley .............................................................................................................................

Norris, Makenzie .........................................................................................................W48

Northoff, Georg ................................................................................................................ 45.1

Novoa, Javier ...............................................................................................

Nucifora, Frederick ......................................................................................... M1 14

Nucifora, Leslie ........................................................................................... M114, T32

Nucifora Jr., Frederick .............................................................................................T32

Nuechterlein, Keith H. .......................................................................W110, W118

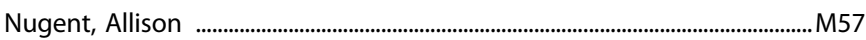

Nungary, John .............................................................................................M134, M198

Nurmi, Erika ...........................................................................................................................

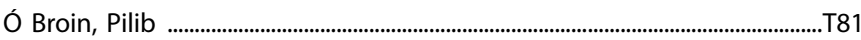

Oathes, Desmond ............................................................................................................ T5

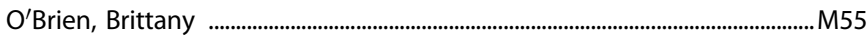

O'Brien, Charles P. ......................................................................................164, W155

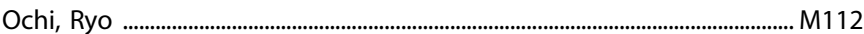

Ochiai, Yukiko .........................................................................................988, M127

Ochs-Ross, Rachel ....................................................................................T75

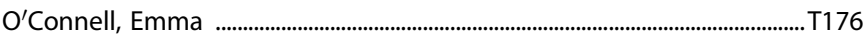

$\mathrm{O}^{\prime}$ Connell, Jeff ......................................................................................................M70

O'Donnell, James ............................................................................................................ W104

O'Donnell, Amy ............................................................................................W110, W1 18

O'Donnell, Kelley .......................................................................................................... T46

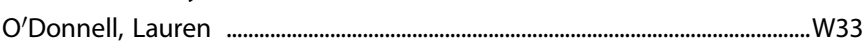


O'Donnell, Patricio …………………………………………………………………… 43.1

Odriozola, Paola .......................................................................................................................

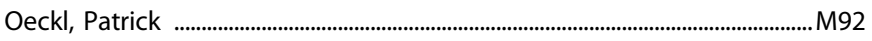

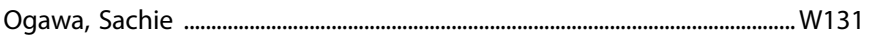

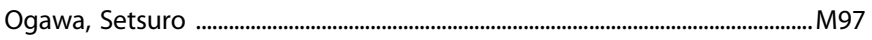

O'Hara, Marguerite .............................................................................................................T93

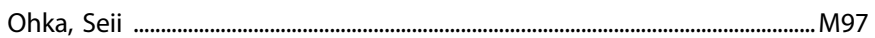

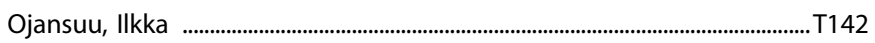

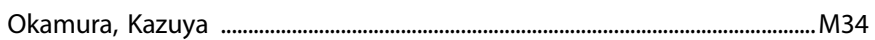

O-Keane, Veronica ........................................................................................................... M92

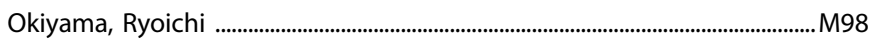

O'Leary, Olivia ...............................................................................................................W89

Olekanma, Doris ......................................................................................................... M177

Olive, M. Foster ...........................................................................................T184, T194

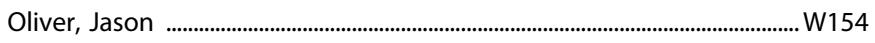

Oliver, Lindsay ............................................................................................. T197, W121

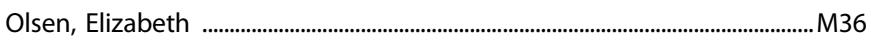

Olson, David ........................................................................................................................... 47.2

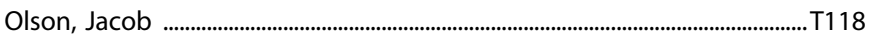

O'Malley, Stephanie ..........................................................................M165, M191, T185

$\mathrm{O}^{\prime} \mathrm{Neal}$, Timothy ........................................................................................................W18

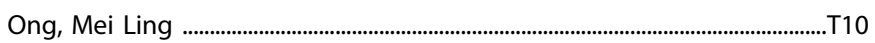

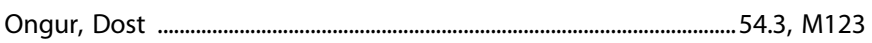

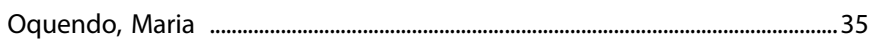

Ordones Sanchez, Evelyn .................................................................M48, T107, T178

Orihuel Menendez, Javier ..........................................................................................T196

Ornelas, Laura ........................................................................................................................W13

Ortinski, Pavel ................................................................................................................. M146

Osanai, Hisayuki .........................................................................................................W131

Ostlie, Noah ..............................................................................................................W179

Oswell, Corinna ...........................................................................................................T54

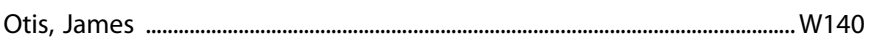

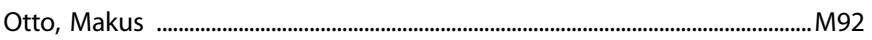

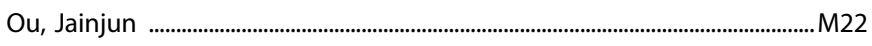

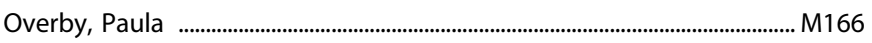

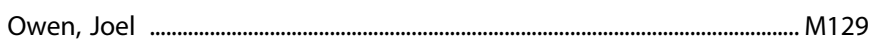

Owens, Lindsey ...................................................................................................................T4

Oxenkrug, Gregory .......................................................................................................M74

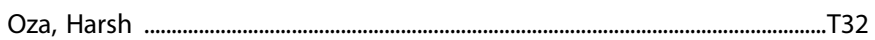

Ozburn, Angela .......................................................................................................W149

Ozenne, Brice .................................................................................................................W77

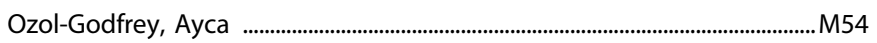

Pachas, Gladys ........................................................................................................... T182

Padilla-Coreano, Nancy .............................................................................100, T118

Pagliaccio, David .................................................................................... T31, T77, T128

Paim Diaz, Alexandre ........................................................................................M64, W57

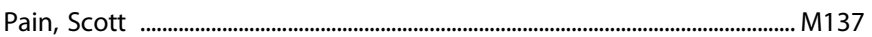

Palka, Jayme ....................................................................................................M78, T117

Pallavicini, Carla .............................................................................................................T58

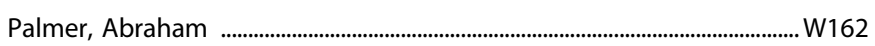

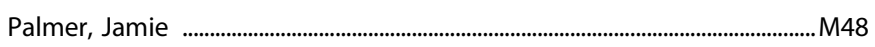

Palumbo, Michelle ..........................................................................................................T11

Pandey, Subhash ...........................................................................................M149, T164

Pandina, Gahan ................................................................................................ T75, W72

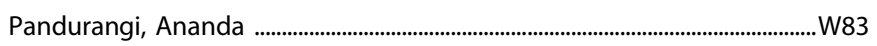

Papakostas, George ................................................................................................W5

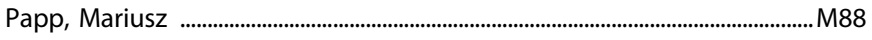

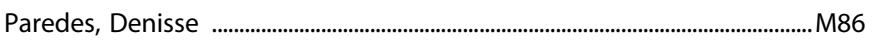

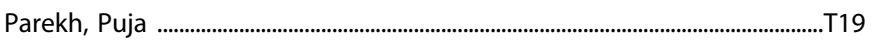

Paret, Christian ...................................................................................................... M105, W65

Paribello, Pasquale .....................................................................................................M38

Parikh, Sagar ..................................................................................................................W74

Parikh, Vinay .......................................................................................................................... W4

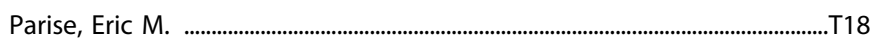

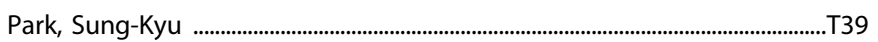

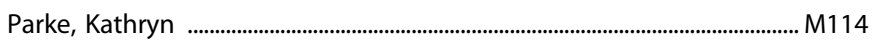

Parker, Ashley ........................................................................................................... M193

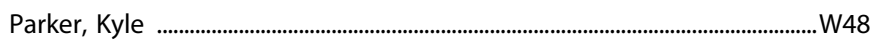

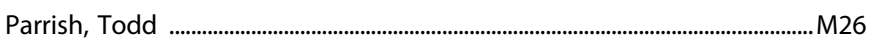

Parvaz, Muhammad ..................................................................................... M154, M156

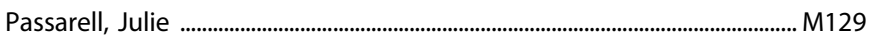

Pasternak, Ofer ...........................................................................................................W137

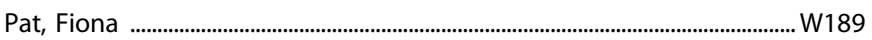

Patarino, Makenzie ......................................................................................................... M100

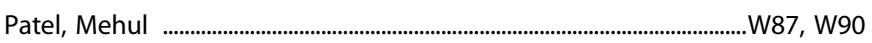

Patel, Reesha ........................................................................................................M100, T9

Pathak, Roma ............................................................................................................... M123

Patt, Marianne .................................................................................................................M73

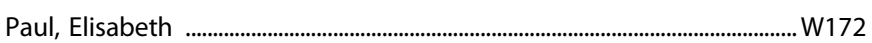

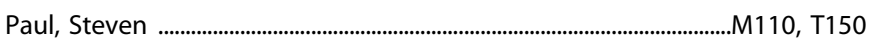

Paulus, Martin ..............................................................44, M25, T61, T73, T200, W84

Pava, Matthew ............................................................................................................ T158

Pavlova, Ina .................................................................................................................. T114

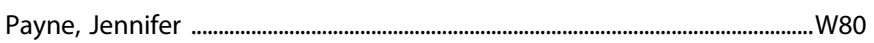

Pearce, Brad ..............................................................................................................W129

Pearlson, Godfrey ..........................................................................42.1, T168, W198

Pecina, Marta ................................................................................................................W76

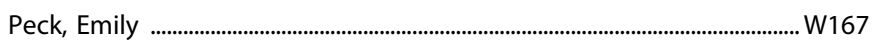

Pedapati, Ernest ................................................................................................ T80, W23

Pedersen, Christian ...................................................................................................T54

Peixoto, Jérémy ............................................................................................................T126

Pena, Catherine ....................................................................................M52, M148, T107

Penalver Bernabe, Beatriz .......................................................................................... 29.3

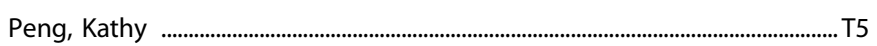

Penninx, Brenda ...........................................................................................................M92

Pennybaker, Steven ....................................................................................................W95

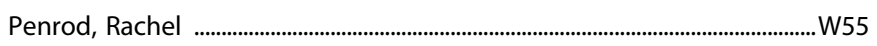

Penzes, Peter ...................................................................................................................... M107

Perez, Stephanie ....................................................................................................W134

Perez-Edgar, Koraly ......................................................................................... 13.1, T84

Perez-Rodriguez, M. Mercedes .......................................11, 33, T159, W43, W191

Pérez-Torres, José ...................................................................................................T130

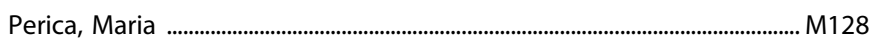

Perich, Matthew G. .......................................................................................................W69

Perino, Michael ................................................................................................................... T6

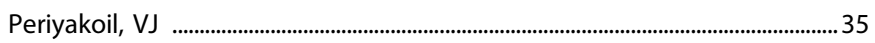

Perkins, Diana O ..........................................................................................................W1 $\mathrm{W} 19$

Perlo, Sarah ........................................................................................................................ M196

Perreault, Melissa .........................................................................................................W135

Perrotta, Katie ..................................................................................................................M11

Peters, Craig ..........................................................................................................M19, T12

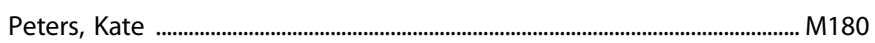


Peterson, Bradley

Peterson, Drew

Peterson, Tim

Petibon, Yoann

Petrakis, Ismene

Petrides, Georgios

Pfeffer, Gerald

M78, T104, W83

Pfeiffer, Ruth

T100

Pfister, Courtney

Pham, Lilyana

T165

Pham, Mimi

....T49

Pham, Vi

...T150

Phan, K. Luan M66, M158, T56

Phelps, Elizabeth

Philip, Noah

Philip, Vivek

Phillips, Karlye

Phillips, Mary

Phillips, Rachel

Piacentino, Daria

Piantadosi, Sean

Picard, Martin

Pickens, Charles

Pieper, Andrew

Pierre Louis, Arline

Pilitsis, Julie

Pina, Melanie

Pine, Daniel

Pinkham, Amy

Pinna, Federica

Pires, Andrea

Pirino, Breanne

Pisanu, Claudia

Piskacek, Simona

Pisoni, Angela

Pittenger, Christopher

Piven, Joseph

Pizzagalli, Diego

Placide, Rebekah

Plano, Andrea

Plasschaert, Ellen

Plessow, Franziska

Plitman, Eric

Plumb, Ashley

Poblete, Guillermo

Pocivavsek, Ana

Podhorna, Jana

Podrebarac, Samantha

Pogorelov, Vladimir

Pohl, Kilian

Polepally, Prabhakar

Politte, Laura

Polizu, Claire

Pollentier, Stephane

Pollock, Bruce

Pomeranz, Lisa
Portis, Samantha

Posey, David

Posner, Jonathan

M59, T102

Post, Anke

M137, W150

Post, Robert

.... 12

Posthuma, Danielle ......................................................................................................... 20.3

Postolache, Teodor ....................................................................................................M70

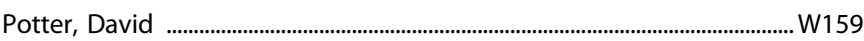

Potter, Kevin ......................................................................................................... T182

Pourdavood, Parham ...............................................................................................W136

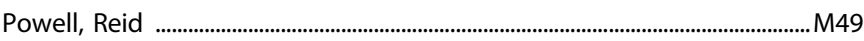

Powell, Susan ..................................................................................................................... W200

Powers, Alexander ....................................................................................................... T150

Pradhan, Amynah ......................................................................................... 32.1, 32.5

Preda, Adrian ............................................................................................................... M130

Preller, Katrin .....................................................................................................4 47.1, 47.4

Prescot, Andrew ............................................................................................................. T122

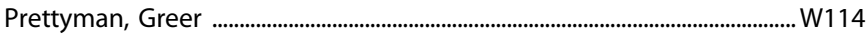

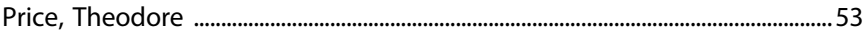

Prisinzano, Tom ................................................................................................................ T124

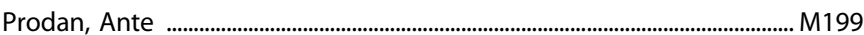

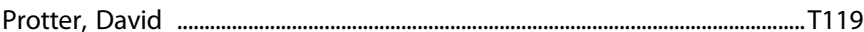

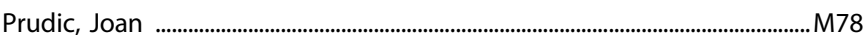

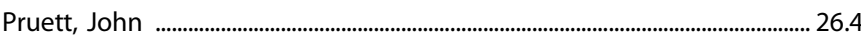

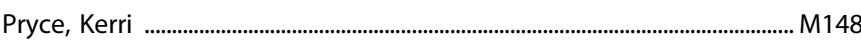

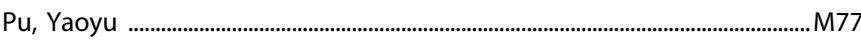

Qazi, Raza ..................................................................................................................W48

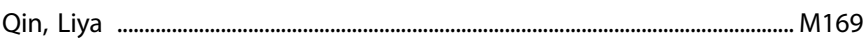

Qu, Youge .......................................................................................................................

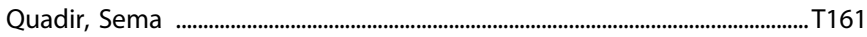

Quarmley, Megan ............................................................................................................M8

Quatrano, Susan ................................................................................................................

Quehenberger, Oswald ............................................................................................T89

Quigley, Jacqueline ...................................................................................................... T193

Quillian, Henry ........................................................................................................... T156

Quintana-Feliciano, Richard ............................................................................................

Quirk, Gregory ......................................................................................... M18, T130

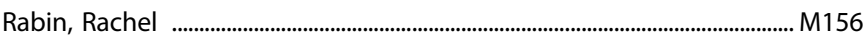

Rabinak, Christine ..........................................................................................M19, T12

Radhakrishnan, Krishnan ..................................................................................... T140

Radhakrishnan, Rajiv ................................................................................................ 43.3

Radke, Anna .................................................................................................................. W179

Radzishevsky, Inna ................................................................................................. M1 18

Rahman, Samir ...................................................................................................... M121

Raio, Candace ............................................................................................................W153

Rajagopal, Lakshmi .................................................................................................. W2

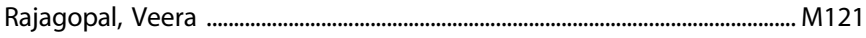

Rajan, Kanaka ............................................................................................................W69

Rajeevan, Nallakkandi .................................................................................................. T140

Rajji, Tarek .............................................................................................. T133, W107

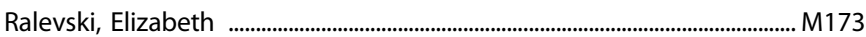

Ralf, Regenthal .............................................................................................M73, W93

Ramaekers, Johannes ..........................................................................................W61

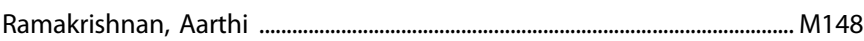

Ramakrishnan, Nithya .......................................................................................... T106

Ramchandani, Vijay ................................................M163, T171, T172, T183, T195 
Ramey, Tanya

M164, T169

Ramjas, Elizabeth

.W43

Ramos-Maciel, Stephanie

...T107

Ramphal, Bruce

...T77

Ramsay, Ian

W116

Ramsey, Christine

...T97

Ramsey, Leslie

M14

Randall, Zoe

T112

Rane, Riddhi

...T87

Rannanpää, Saara

M68

Rao, Nikhil

W109

Rasizer, Lindsay

W179

Rassnick, Stephanie ......................................................................................................W72

Rauh, Virginia ..............................................................................................................T77

Ravi, Meghna ............................................................................................................... T3

Ravichandran, Caitlin ................................................................................................T11

Rawls, Eric

Ray, Lara

M152, T42, W181

Raznahan, Armin

Reavis, Zackery

Rebola, Nelson

T126

Reda, Mariam

W17

Regier, Paul

M164

Regnier, Grace

... W5

Reichel, Carmela

....M3

Reichenberg, Abraham

T198

Reid, Meredith

..T69

Reif, Andreas

M92

Reigle, Jame

W56

Reilly, Erin

....T88

Rein, Ben

...T40

Reiner, Benjamin

25.2

Reiner, David

T196

Reininghaus, Ulrich

Reiss, Allan

Reissner, Kathryn J.

36.5, T163

Remington, Gary

T37, W45

Renshaw, Perry

T79, T122

M167

Rentsch, Christopher

..T160

Rentschler, Katherine

..14, 14.5, 18, M18, T1, T3, T118, W9, W17, W78

Ressler, Kerry

M139, T157

Reyderman, Larisa

W175

Rice, Kenner

W196

Riceberg, Justin

M145

Richard, Jocelyn

M13

Richard-Malenfant, Claude

...T96

Richards, Christine

W112

Richardson, Charles M.

M141

Richie, Christopher

Rieger, Michael

M80, W92

Rill, Rudriger

Rilling, James .................................................................................................................W26

Rincón-Cortés, Millie ................................................................................................... 34.5

Rios, Beatriz

M24

Risbrough, Victoria

M2, M195, T72
Risso, Stefania .......................W105

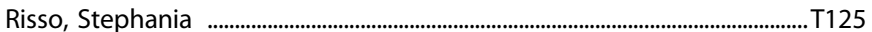

Ritchie, Jobe ................................................................................................................. W187

Riva Posse, Patricio ........................................................................................................W83

Rizzoni, Elizabeth ........................................................................................W137

Roach, Brian .............................................................M130, T34, W95, W119, W136

Roalf, David .......................................................................................................T59

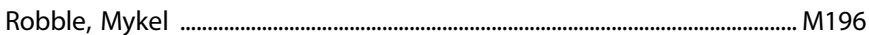

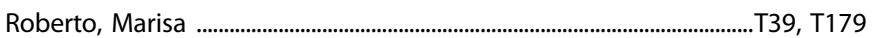

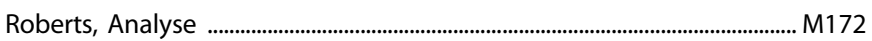

Roberts, Benjamin ....................................................................................................... M198

Roberts, Rosalinda ................................................................................................T147

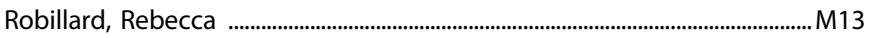

Robinson, Benjamin ............................................................................................

Robinson, Delbert ...................................................................................................W127

Robledo, Renato .............................................................................................................

Roche, Daniel ......................................................................................................... M152

Roche, Joy .................................................................................................................. T147

Rock, Rachel ............................................................................................................ M100

Rocks, Devin ...................................................................................................... W142

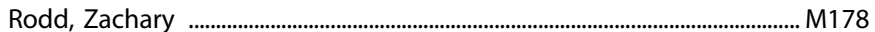

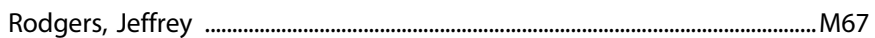

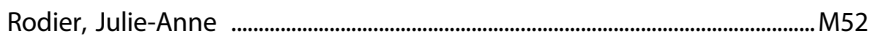

Rodriguez, Carolyn ..................................................................................................... 33

Rodriguez, Lionel ...........................................................................................................20

Rodriguez-Romaguera, Jose ................................................................................. 14.3

Roeske, Maxwell .............................................................................................T154, W133

Roffman, Joshua ....................................................................................................W31

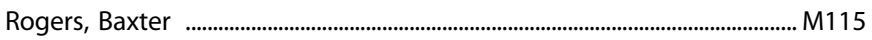

Rogers, Charleanne ...........................................................................................T178

Rogers, Hopewell ………………………………………………………………….... M24

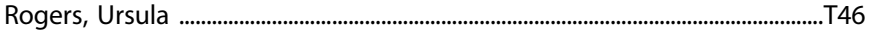

Rokham, Hooman ........................................................................................................... M122

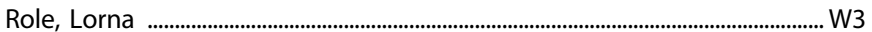

Rolin, Donna ..............................................................................................................W90

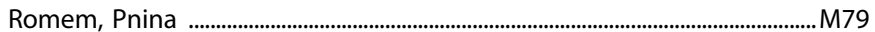

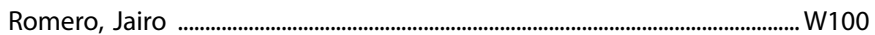

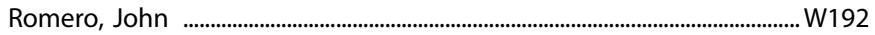

Romero, Maria Celeste ..........................................................................................T58

Romero-Medrano, Lorena .....................................................................................W43

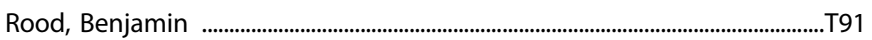

Ropchan, Jim ...............................................................................................T45, W171

Rosell, Daniel ........................................................................................................W191

Rosen, Bruce ..........................................................................................................W16

Rosenblum, Katherine .......................................................................................T167

Rosenbrock, Holger ..............................................................................................T135

Rosenquist, Peter ........................................................................................................... M78

Roshgadol, Jacob ......................................................................................................T19

Ross, Christopher .....................................................................................................T32

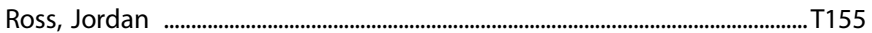

Rossi, Mark ....................................................................................................................T54

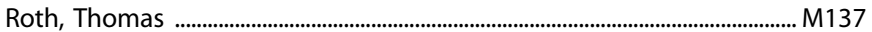

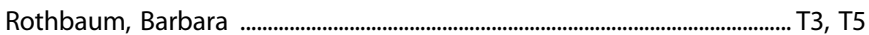

Rothschild, Anthony ................................................................................W74, W128

Rothwell, Patrick ....................................................................................................... 10.3

Rotrosen, John .....................................................................................................T4 
Rotstein, Natalie

Roussos, Panos

Rowland, Laura

Rowley, Helen

Rozalski, Vincent

Ruan, Hongyu

W12, W16

Ruan, Qiu

T174, W161

Rubin, Leah

... W198

Rubinow, David R.

M80, W92

Rudebeck, Peter

W196

Rudolph, Uwe

M84

Rujescu, Dan

.M81

Rullmann, Michael

....M73

Rumbaugh, Gavin

M143

Ruparel, Kosha

T59, W114

Rush, A. John

...W68

Rushmore, Jarrett

W137

Rusowicz, Aleksandra

Russell, Justin

M12

Russell, Trinity

M141

Russo, Scott

Ryan, Karen M.

Ryan, Neal

W102

Ryan, Niamh

M186

Ryan, Saoirse

....T128

Rynn, Moira

....W72

Saad, Ziad

...T161

Sabino, Valentina

..M73

Sabri, Osama

...W70

Sacchet, Matthew

.M73, W93

Sacher, Julia

M119, W196

Sadahiro, Masato

W185

Saddoris, Michae

Sadreyev, Ruslan

T13, W39

Saggar, Manis

...T113

Shy, Katalin

...... 18

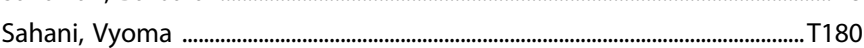

Sahin, Olcay Senay ....................................................................................................W137

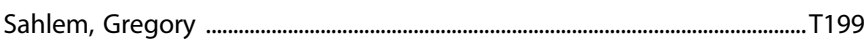

Saijo, Masayuki ..................................................................................................................

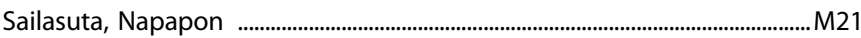

Sajatovic, Martha ............................................................................................... $31,31.2$

Sakloth, Farhana ......................................................................................................... M148

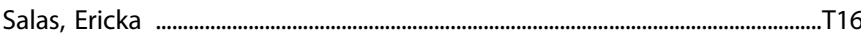

Salas, Ramiro .................................................................................................M72, T131

Salmeron, Betty Jo ................................................................................................... M151

Salome-Sanchez, Charles ......................................................................................... M94

Salum, Giovanni

Salvadore, Giacomo

28.3, W72, W110

Sam, Alinna

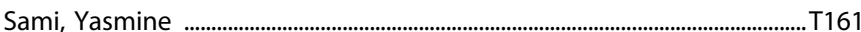

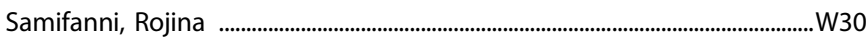

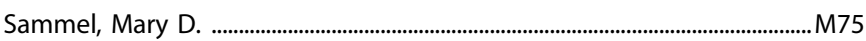

Samuels, Benjamin .........................................................................................................M62

Sánchez-Navarro, Marcos

...T130
Sand, Michael

$8.7,21$

Sander, Christin

W166

Sanders, Kevin

W101

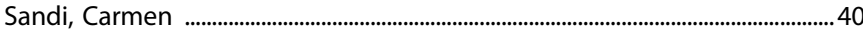

Sands, Lester ..................................................................................................... M182

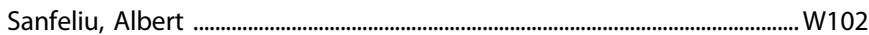

Sanghani, Sohag _................................................................................................... T104

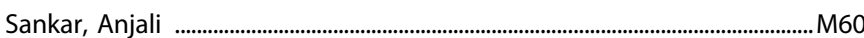

Santhumayor, Brandon ........................................................................................... W7

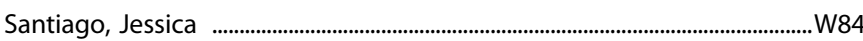

Sarabdjitsingh, Angela ...........................................................................................W78

Sarac, Cansu ..........................................................................................................W120

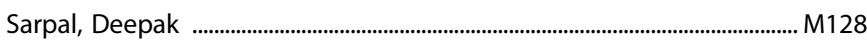

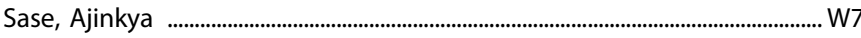

Satheesh, Agarsh .......................................................................................................W30

Satlin, Andrew .........................................................................................T110, T148

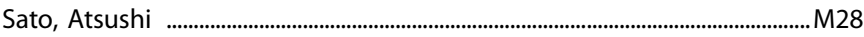

Satterthwaite, Theodore ...........................................................................T59, W114

Saudagar, Vikram ..........................................................................................................W79

Saunders, Benjamin .............................................................................................. W1

Savitz, Adam .................................................................................................W60

Sawa, Akira ....................................................................................... 114, T32, W80

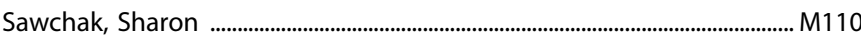

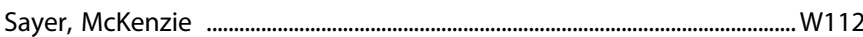

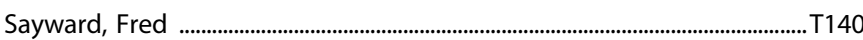

Scaini, Giselli .......................................................................................................................W57

Scangos, Katherine .................................................................................................... 46.4

Schaeffer, Andrea .........................................................................................................W37

Schafer, Matthew ............................................................................................M154, T180

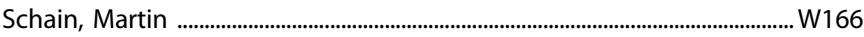

Scharrer, Ulrike ............................................................................................................W93

Schatzberg, Alan ....................................................................................... 11, 33, W67

Scheinost, Dustin .......................................... M191, W27

Schermitzler, Brandon ...........................................................................................W1 W

Schieber, Jennifer ..................................................................................................... T40

Schifani, Christin ................................................................................................. T133

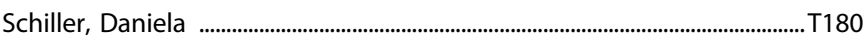

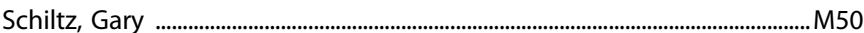

Schindler, Emmanuelle .................................................................................................. 47.5

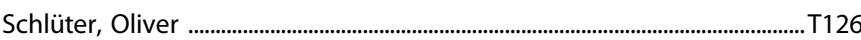

Schmack, Katharina ...........................................................................................................6

Schmahl, Christian ............................................................................................ M105, W65

Schmidt, Mathias V. .................................................................................................W78

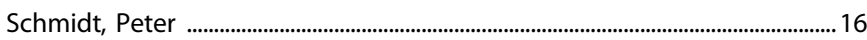

Schmitt, Andrea .................................................................................................................

Schmitt, Lauren ................................................................................................. T80, W23

Schmitt, William ..........................................................................................................T182

Schneeberger-Pane, Marc ............................................................................................ M43

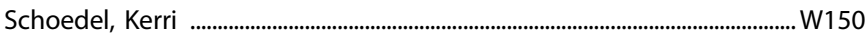

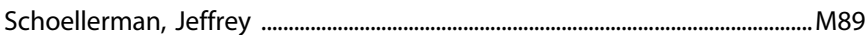

Schoenfeldt-Lecuona, Carlos ...................................................................................M92

Schroder, Hans .............................................................................................. M195, M196

Schroeder, Heidi ................................................................................................................T70

Schroeder, Rachel .......................................................................................................W98

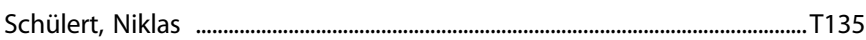

Schultheis, Christian ……………………………………………………….......T135, W65 
Schultz, Timothy

W103

Schulz, Andreas

...M92

Schulz, Kurt

W191

Schurdak, Jennifer

W197

Schwandt, Melanie

T172, T183, T195, W151, W156

Scofield, Michael

M144, W143

Scolnick, Ed

Scott, Madeline

.136

Scott, McRae

M106, W125

Seboek Kinter, Dalma

M96

Sebra, Robert

M137, W150

Sedighim, Sharona .......................................................................................................... M170

Seethaler, Magdalena ............................................................................................ W137

Sege, Christopher ..................................................................................................T5

Seidling, Kailyn ............................................................................................................ M150

Seiger, Rene ................................................................................................................. M81

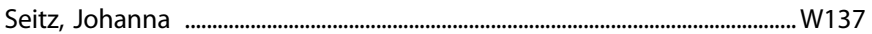

Sekar, Shwetha ....T71

Seligowski, Antonia

M18, W17

Selimbeyoglu, Aslihan

M41, M140

Seminowicz, David

.T62

Seney, Marianne

$37.1,37.4$

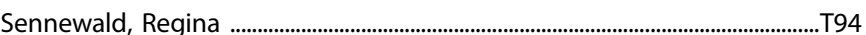

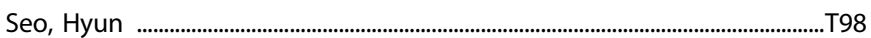

Seok, Darsol .........................................................................................................................W64

Sepulveda, Yadira ................................................................................................................. M39

Sepulveda-Orengo, Marian .....................................................................................W169

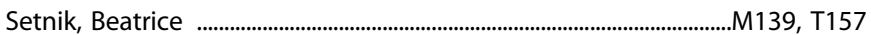

Severino, Giovanni ........................................................................................................

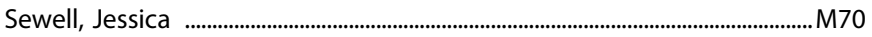

Shafer, Robin .............................................................................................................T8

Shaffer, Rebecca ...........................................................................................................W23

Shah, Abhishek

....T29

Shaham, Yavin 22, M141, M142, T196

Shahbo, Ellie M118

Shaik, Riaz

M156, W120

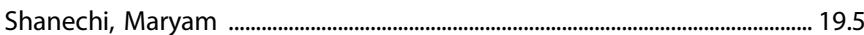

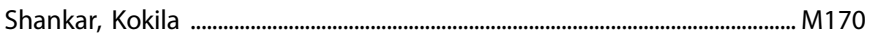

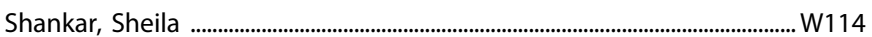

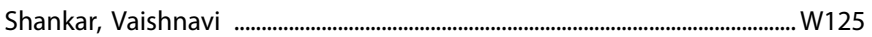

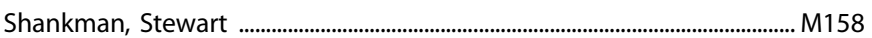

Shanley, Mary Regis ....................................................................................................... M51

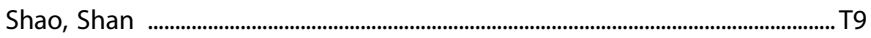

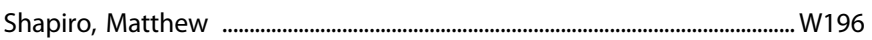

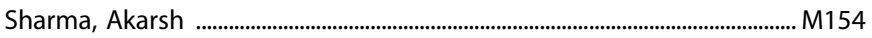

Sharma, Kamakashi ............................................................................................... M132

Sharma, Vikas ...............................................................................................................W65

Sharp, Andrew ............................................................................................... M120

Sharp, Richard ................................................................................................M198, T81

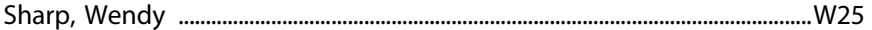

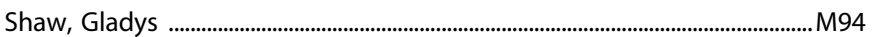

Shaw, Philip ..................................................................................W25

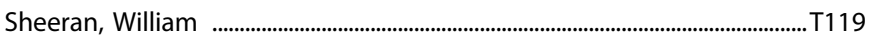

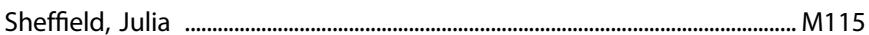

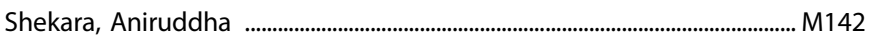

Sheline, Yvette
Shelton, Richard

W59, W74

Shen, Li

M148, T18

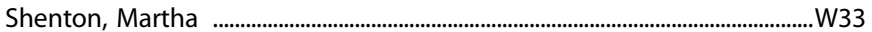

Sherafat, Yasmine ................................................................................... M183

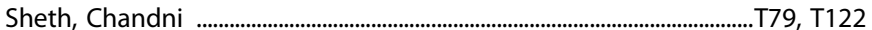

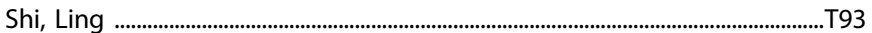

Shi, Tracey ...............................................................................................................T31

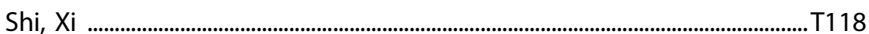

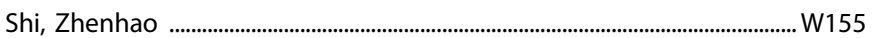

Shih, Pei-an (Betty) .......................................................................................................T89

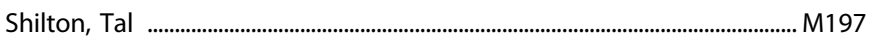

Shin, Jaemin ................................................................................................................ T3

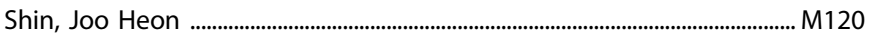

Shinohara, Russell ..................................................................................................T59

Shlik, Jakov ............................................................................................................

Shokri Kojori, Ehsan …………………………………………......M159, T171, W156

Shokri-Kojori, Ehsan ................................................................................W151, W173

Short, Annabel K. ................................................................................................W28

Shrestha, Sharon ........................................................................................................T13

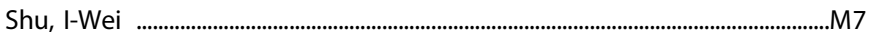

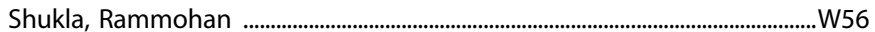

Shuldiner, Alan ....................................................................................................................M70

Shvartzman, Pesach ..........................................................................................................M79

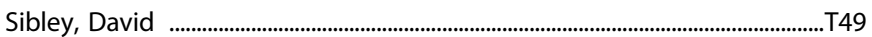

Siciliano, Cody ............................................................................................M16, T118

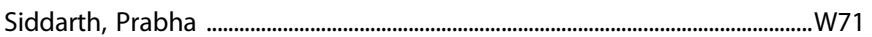

Siegle, Greg J. .....................................................................................................................W8

Sikich, Linmarie .........................................................................................................W10

Silamongkol, Thanharat ............................................................................................

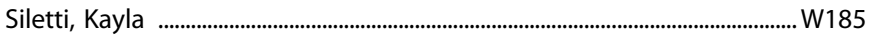

Silk, Jennifer ..........................................................................................................T84

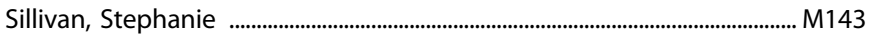

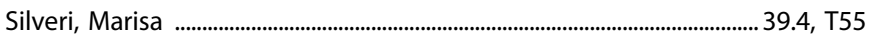

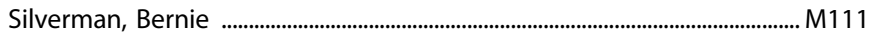

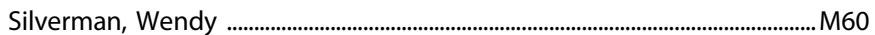

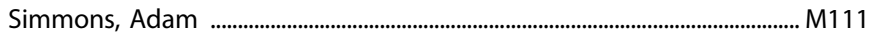

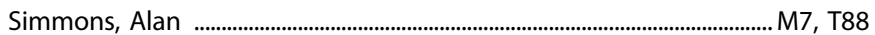

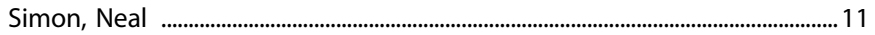

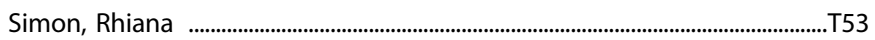

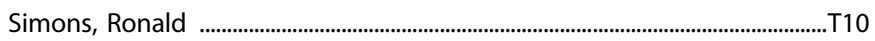

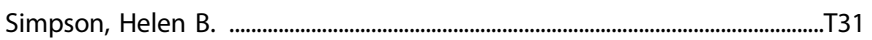

Simpson, Sierra ............................................................................................................. M168

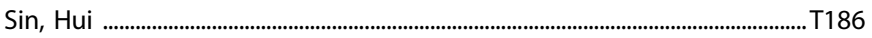

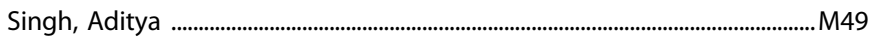

Singh, Mahendra ................................................................................................... M133

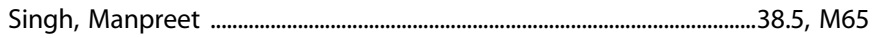

Sinha, Rajita ............................................................................................................. 34.2

Skipper, Jamie ............................................................................................. T102

Slate, Sarah Rose .........................................................................................................T67

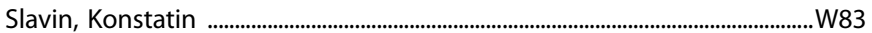

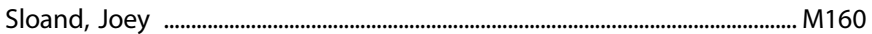

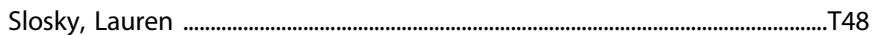

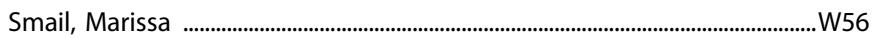

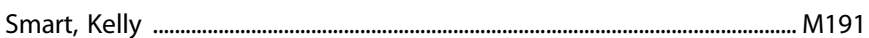

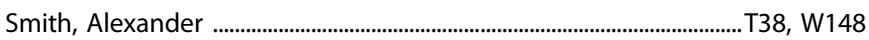

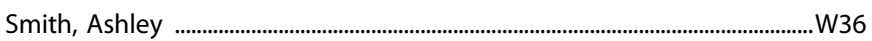


Smith, Brittany

Smith, Caroline

Smith, David

Smith, Emma

Smith, Eric ...

Smith, Janice

Smith, Jared

Smith, Lauren

Smith, Mark A.

Smith, Robert

Smith, Sharon

Smoller, Jordan

Smucny, Jason

Sneddon-Yepez, Elizabeth

Soares, Jair

Soehner, Adriane

Sogos, Valeria

Soja, Jacqueline

Solberg Woods, Leah

Song, Hongjun

Sonnenschein, Susan

Sood, Ankit

Soontornniyomkij, Benchawanna

Sorensen, David

Soriano, Esmeralda

Sortman, Bo

Soto-Muniz, Yanilka

Soula, Anais

Southwick, Steven

Spanagel, Rainer

Spann, Marisa

Speier, William

Spell, Laura

Spencer, Linda

Spencer, Sade

Spencer-Salmon, Camille

Spodnick, Mary

Sponheim, Scott

Sprock, Joyce

Squassina, Alessio

Srinivasan, Nitin

Stackmann, Michelle

Staglin, Brandon

Stahl, Stephen

Stangl, Bethany

Stankovic, Srdjan

Stanley, Barbara

Steinacker, Petra

Steiner, Johann

Steiner, Michel

Steiner, Steiner

Stenbæk, Dea S.

Stensson, Niclas

Stepanova, Ekaterina

Stephan, Clifford

Stern, Sarah
Stevens, Benson

Stevens, Hanna

Stevens, Jennifer

Stevens, Michael

Stewart, Jennifer

Stewart, Kent

Stigler, Kimberly

Stoct, Janet

Stoddard, Joel ..

Stogios, Nicolette

Straub, Richard

Strawn, Jeffrey

Strege, Marlene

Stricklin, Ashley

Stringfield, Sierra

Stuber, Garret

Sturman, Oliver

Su, Yujuan

Subar, Anni

Subotnik, Kenneth L.

Sudre, Gustavo

Suessmuth, Sigurd D.

Suhandynata, Ray

Sulima, Agnieszka

Sullivan, Anna

Sullivan, Kate

Summergrad, Paul

Sun, Hui

Sun, Kevin

Sun, Lei

Sun, Yuting

Suomi, Stephen

Süssmuth, Sigurd

Sutherland, Ashley

Suthoff, Ellison

Sutton, Brianne

Suvisaari, Jaana

Suzuki, Kanzo

Suzuki, Masako

Svensson, Kjell

Swafford, Austin

Swain, James

Swann, Alan

Swanson, Devin

Swartz, Holly

Swartzwelder, H. Scott

Swearingen, Hannah

Sweeney, John A.

Sweet, Robert

Sweitzer, Maggie

Swerdlow, Neal

Sylvester, Chad

Synan, Colleen

Szatmari, Peter

Szcezepankiewicz, Filip A.

Szeszko, Philip
M163

...W98

T1, T3, W9, W17

.T168

.T61

..T49

..T11

...W64

$\mathrm{T} 21, \mathrm{~T} 22$

.....T37

M120

....T70

...W85

M114

M157

.....T53

..... W8

M170

.....T27

W110

...W25

...M92

... M39

W175

..T65

...W87

M74

T179

...W39

W126

..T100

.....T83

.T135

.T103

..T109, W91

....W46

.T142

... M53

W142

... W2

W130

..T167, W158

11, M55, T106

...M88

...M60

M178

...M10

T70, T80, W23, W198

M107, T33, T132, W124

M153

M134, M198, W100

...13.2, T6

...T149

... M21

W137

W127 
Szombathelyi, Zsolt

Szpunar, Mercedes

Szumlinski, Karen

T174, T175

Taccheri, Giulia M119

Tadayon-Nejad, Rez

Taffe, Michael

Tagliazucchi, Enzo

Taipale, Heidi

158

Tajinda, Katsunori

...M68, T142

Takahashi, Kazushi ...T81

Takamatsu, Yukio M98

Takeuchi, Hiroyoshi ... M28

Talati, Ardesheer

M83, T37

Talledo, Jo

... M59, T102

Talmage, David

M198, W100

Tamminga, Carol

.... W3

Tan, Bowen

T25, T117, W198

Tan, Laura

M43

Tan, Yunfei

T151

Tanaka, Miho

M77

Tang, Sunny

M28

Tang, Victor W121

Tani, Hideaki ....T92

Taniguchi, Makoto

M21, M83, W128

T162, W145

Taniguchi, Masayuki ........................................................................................................ M76

Tanner, Julie-Anne M69

Tanskanen, Antti

M68, T142

Tao, Ran . M71

Tapert, Susan

50, W34

Tapia, Cynthia M.

M49

Tapia, Melissa

W48

Targum, Steven

M110

Tarumi, Ryosuke

M112

Tat, Jennifer

M16

Taylor, Anna

.M99

Taylor, Bonnie

W24

Taylor, Danielle ....T57

Taylor, Jerome

Taylor, Matthew

8.11

M164

Taylor, Stephan

45, 45.3, W83

Taylor, Valerie ....T37

Teed, Adam ....T73

Teklehaimanot, Abeba .............................................................................................. M78

Tennen, Howard

M173

Teresi, Giana

M65

Tessari, Michaela

W150

Testen, Anze

$\mathrm{T} 163$

Teyler, Timothy

....T34

Thai, Michelle

M35

Thamer, Claus

W65

Thames, April

1, W82

Thang, Loc ... M171

Thase, Michael W59, W74

Therman, Sebastian T142

Theunissen, Eef W61

Thibeault, Kimberly
Thom, Robyn ..T11

Thomas, Adam

Thomas, Christopher

M140

Thomas, Jennifer

...T15

Thomas, Mark .

..T51

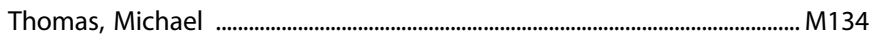

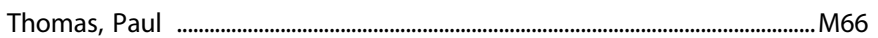

Thomashow, Katherine .............................................................................................W8

Thompson, Allison .......................................................................................................... T5

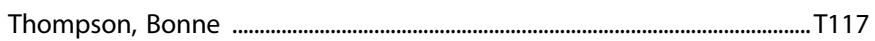

Thompson, lan .........................................................................................................T65

Thompson, Ronald ...........................................................................................W164

Thompson, Scott ................................................................................M84, W97, W99

Thompson, Wesley .....................................................................................................T73

Throop, Alanah ..........................................................................................................T92

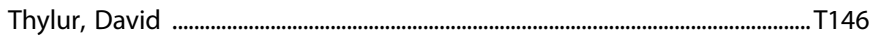

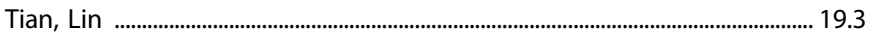

Tieu, Lani .............................................................................................................M39, M170

Tiihonen, Jari ....................................................................................................M68, T142

Tillage, Rachel .............................................................................................................W22

Timmermann, Christopher .......................................................................................T58

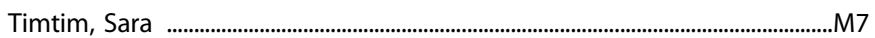

Tippani, Madhavi ......................................................................................................W10

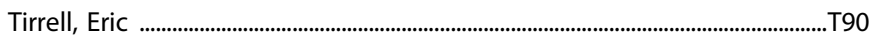

Tishler, Todd A. ........................................................................................W110, W1

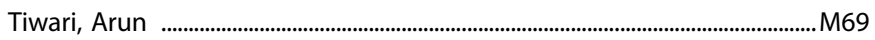

Tobe, Russell ...............................................................................................................W10

Toeroek, Michael .........................................................................................................W150

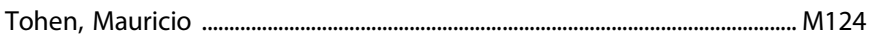

Tokuno, Shin-ichi ..................................................................................................T81

Tollkuhn, Jessica ............................................................................................................ T107

Tomandl, Josef ......................................................................................................W137

Tomasi, Dardo .......................................................M159, W47, W151, W156, W173

Torregrossa, Mary ....................................................................................M157, W146

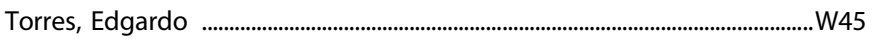

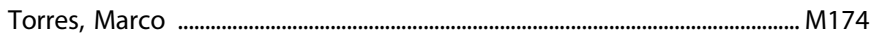

Torres-Berrio, Angelica ......................................................................................... M148

Torres-Berrío, Angélica .................................................................................................T18

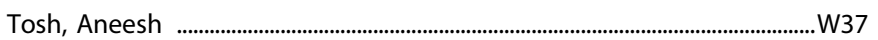

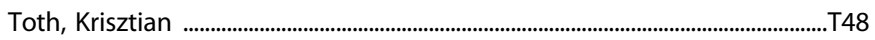

Townsend, Reid ....................................................................................................... M171

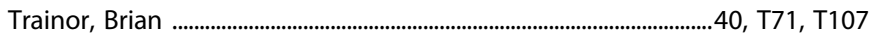

Tran, Matthew ........................................................................................................W109

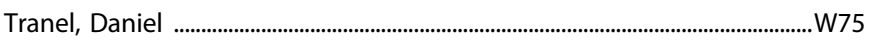

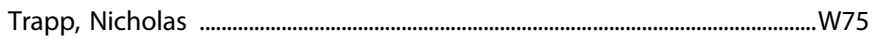

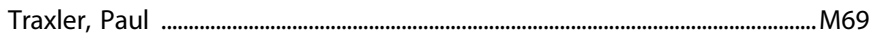

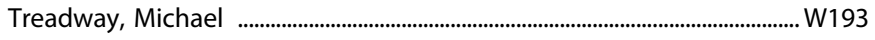

Tregellas, Jason .............................................................................................................W46

Tritschler, Laurent .............................................................................................................T29

Trivedi, Madhukar ................................................................1, T68, W57, W59, W199

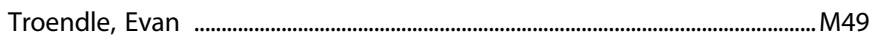

Trontti, Kalevi .......................................................................................................... T142

Tropea, Daniela ........................................................................................................W102

Troppoli, Timothy ........................................................................................M84, W99

Trotter, Claudia ......................................................................................................W140

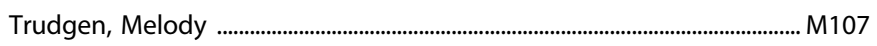




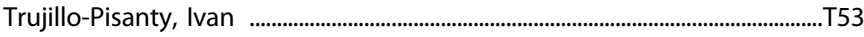

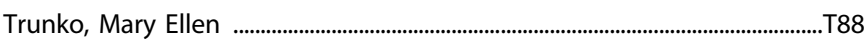

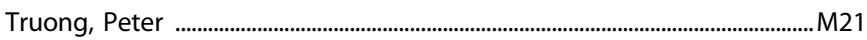

Tsai, Joyce ......................................................................................................................... M54

Tse, Yiu Chung ................................................................................................................W66

Tseng, Angela .............................................................................................................T78

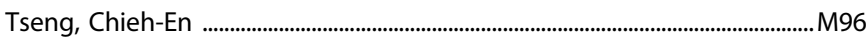

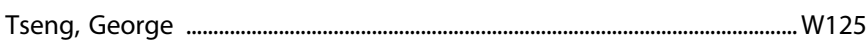

Tseng, Wan-Ling ...............................................................................................................M4

Tsuang, Ming T ..........................................................................................................W1 W

Tsugawa, Sakiko ...........................................................................................M93, M112

Tsvetkov, Evgeny ...................................................................................................... M30

Tunstall, Brendan ..............................................................................................167, T179

Tuominen, Lauri .......................................................................................................... 13

Turk-Brownes, Nicholas .........................................................................................W64

Turkoz, Ibrahim ............................................................................W62, W110, W118

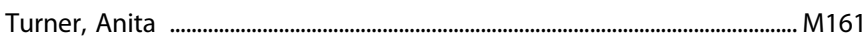

Turner, Jessica .................................................................................................................... 42.4

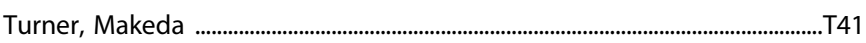

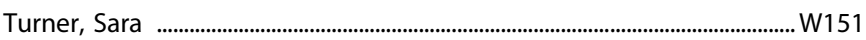

Turner, Victoria ...........................................................................................................T74

Tye, Kay .............................................................................41.1, 41.2, M100, T9, T1 18

Tyrka, Audrey .............................................................................................................T9

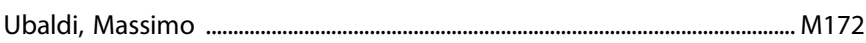

Uchida, Hiroyuki ...............................................................................M83, M112, W128

Uchino, Shigeo ..............................................................................................................

Ufer, Mike ........................................................................................................................ W150

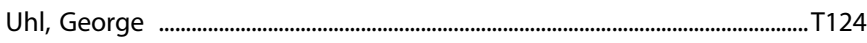

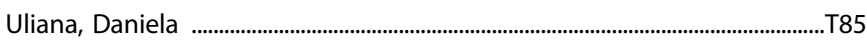

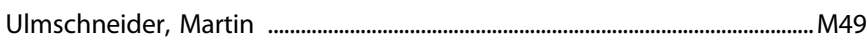

Underhill, Suzanne .........................................................................................................W6

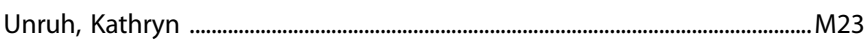

Upshaw, Valerie ...........................................................................................................T73

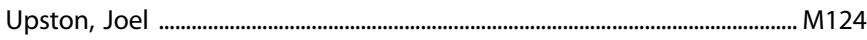

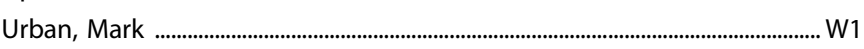

Urbano Blackford, Jennifer .................................................................................. M115

Ustohal, Libor ...............................................................................................................W137

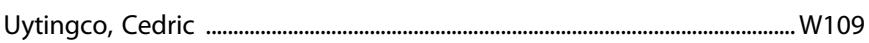

Vaccaro, Lucio ...................................................................................................................W143

Vadnie, Chelsea .........................................................................................................W163

Vadukapuram, Ramu ................................................................................................. T141

Vaessen, Thomas ......................................................................................................W117

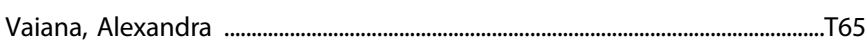

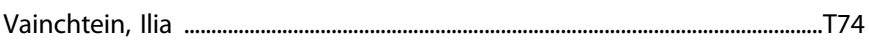

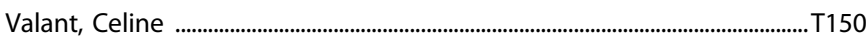

Valerino, Jessica ....................................................................................................... T156

van Aubel, Evelyne .............................................................................................W117

Van Booven, Derek .................................................................................................W147

Van Der Ark, Peter .......................................................................................................W60

Van der Veen, Jan-Willem .....................................................................................W151

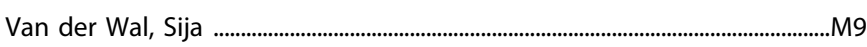

van Dijk, Milenna .............................................................................................................M59

Van Doren, Eliyana ......................................................................................................... T175

Van Eck, Kathryn ........................................................................................................W50

van Erp, Theo ............................................................................................................. M130

van Grootheest, Gerard ..................................................................................................M92 van Leeuwen, Judith ...............................................................................................T60

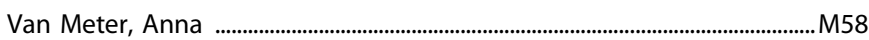

van Rooij, Sanne ................................................................................................ T1, T3

van 't Wout, Mascha .......................................................................................M14, W10

Van Voorhies, Kalynn ............................................................................................W13

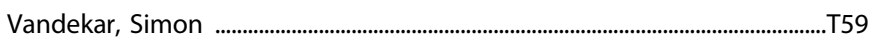

Vangel, Mark ...........................................................................................W33, W137

Vannan, Annika ............................................................................................................ T184

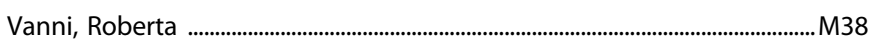

Vanover, Kimberly ......................................................................................... T110, T148

Varghese, Nimmy ........................................................................................................W61

Varney, Mark .................................................................................................................... W1

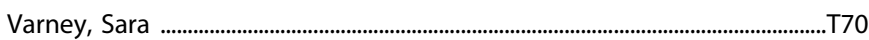

Vasaturo-Kolodner, Talia ...................................................................................... T186

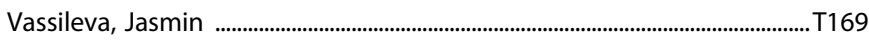

Vasudevan, Anju ........................................................................................................ M1 18

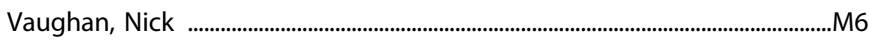

Vaurio, Olli ................................................................................................................... T142

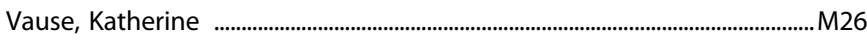

Vawter, Marquis ............................................................................................5. W4.2, W126

Vázquez-Baeza, Yoshiki ...........................................................................................W130

Veenema, Alexa ............................................................................................... 40, M32

Veenstra-VanderWeele, Jeremy ..................................................18, M101, W101

Velázquez-Díaz, Coraly ............................................................................................. T130

Velisek, Haley A. ...........................................................................................................

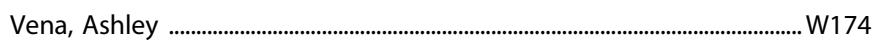

Vendruscolo, Janaina ............................................................................................W151

Vendruscolo, Leandro .................................................................M167, T179, W151

Venkatesan, Sridevi ..................................................................................................W5

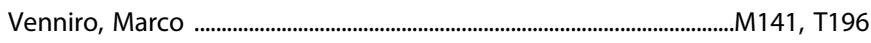

Ventura, Joseph ....................................................................................................W118

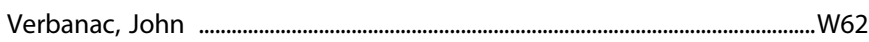

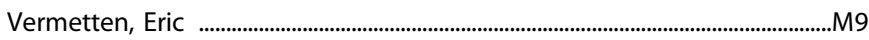

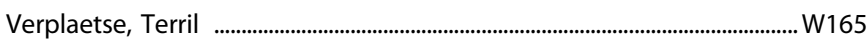

Viardot, Geoffrey .............................................................................................................. T135

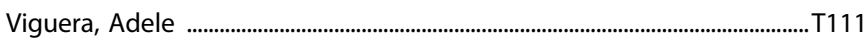

Vijay, Aishwarya .........................................................................................................W157

Villalobos-Manriquez, Francisca ............................................................................W8

Villringer, Arno ...................................................................................................M73, W93

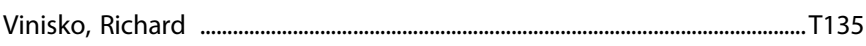

Vinkers, Christiaan .......................................................................................................... T60

Vinson, Tonette ......................................................................................................... T195

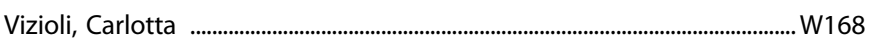

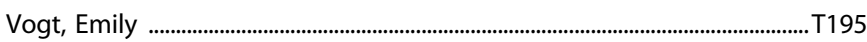

Voineskos, Aristotle ............................................... M109, T133, T197, W121, W128

Voineskos, Daphne ...................................................................................T92, T153

Vojtisek, Lubomir ...............................................................................................W137

Vo-Le, Brittany .................................................................................................................T106

Vo-Le, Bylinda ...................................................................................................T106

Volkow, Nora ..............................16, 50, M159, T171, W47, W151, W156, W173

Voloudakis, Georgios ............................................................................................... 121

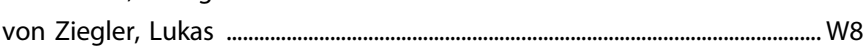

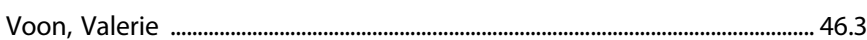

Vuijk, Pieter ...................................................................................................................W W31

Vyas, Gopal R. ...............................................................................................................W W1

Waag, Rebecca .......................................................................................................................... W8 


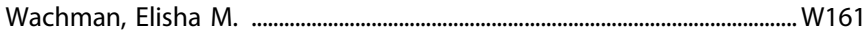

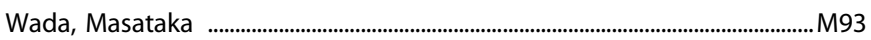

Wadsworth, Annie ....................................................................................................... M49

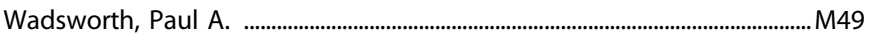

Wagner, Ariella ............................................................................................................. M156

Wagoner, Ryan ................................................................................................................ T101

Wahlestedt, Claes .................................................................................................W147

Waiess, R. Aaron ...................................................................................................... M178

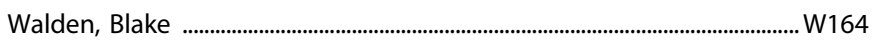

Walicki, Marie ............................................................................................................W48

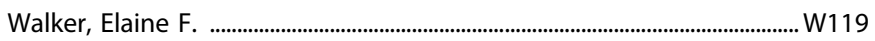

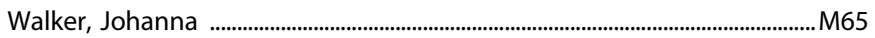

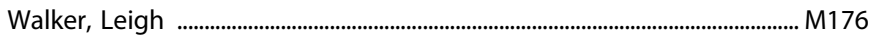

Walker, Shakeera ...................................................................................................T123

Wallace, Art ...............................................................................................................W95

Wallace, Grant ..............................................................................................................W64

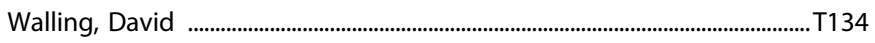

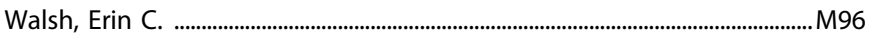

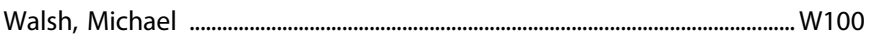

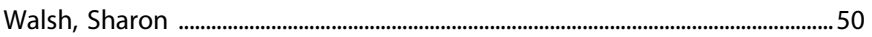

Walters, Jennifer .......................................................................................................W18

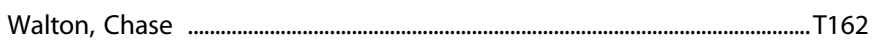

Waltz, James ................................................................................................................ W117

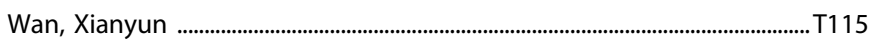

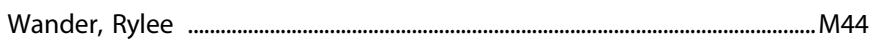

Wang, Daniel ....................................................................................................................W160

Wang, David ..................................................................................................................W37

Wang, Gene-Jack ...................................... M159, T171, W47, W151, W156, W173

Wang, Han ................................................................................................................... T163

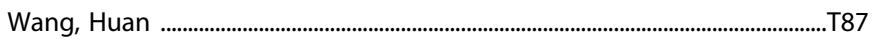

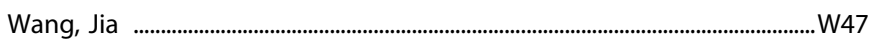

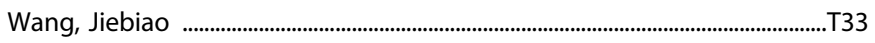

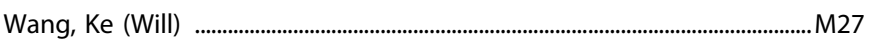

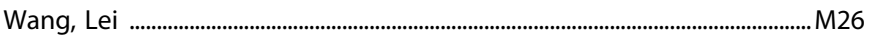

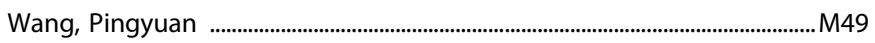

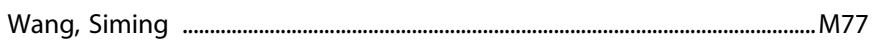

Wang, Wei ............................................................................................M109, T124, T133

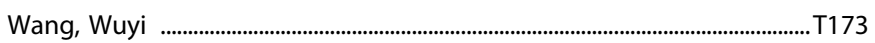

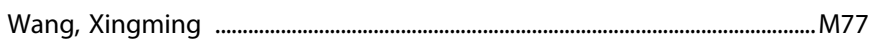

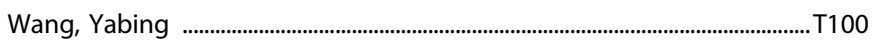

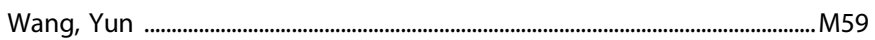

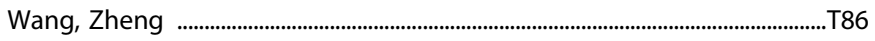

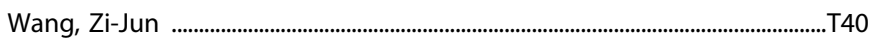

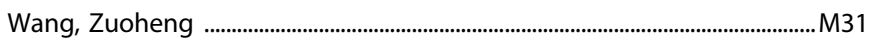

Wanna, Cassandra ..............................................................................................W17

Ward, Heather ............................................................................................................W138

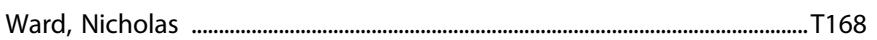

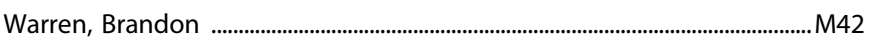

Warthen, Katherine G. .............................................................................................M56

Waterhouse, Barry .......................................................................................................T191

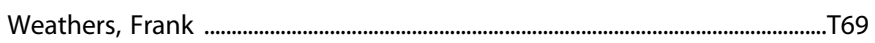

Weber, Lukas ..................................................................................................................... W109

Weddington, Javier ..................................................................................................... M100

Weera, Marcus ....................................................................................................................M5

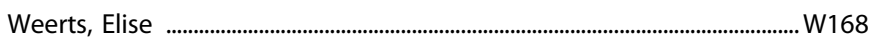

Wei, Heather .............................................................................................................W5

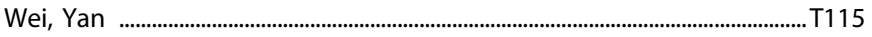

Wei, Yue ................................................................................................................. T33

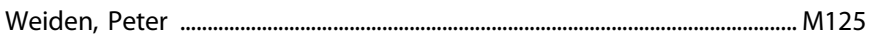

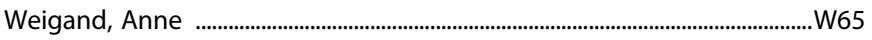

Weinberger, Daniel R. .................................................................................M71, M120

Weiner, Richard ............................................................................................................M78

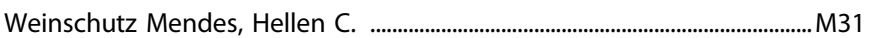

Weinshenker, David ......................................................................................W22, W144

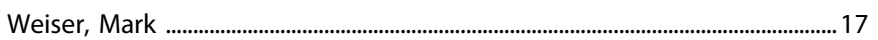

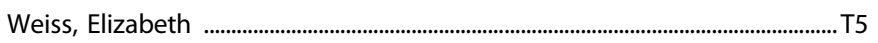

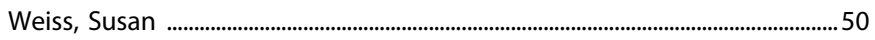

Weissman, Myrna .........................................................................................M59, T102

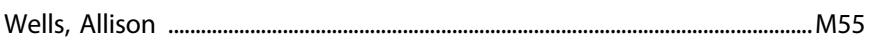

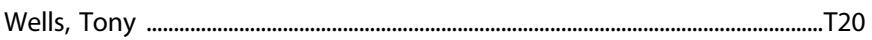

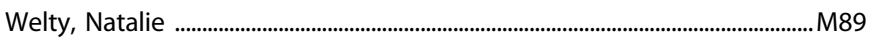

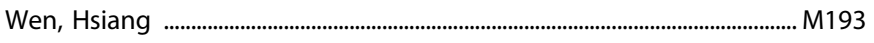

Wengler, Kenneth ................................................................................W122, W153

Wenzel, Jennifer ................................................................................................................ 22

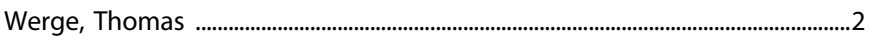

Werneberg, Brian ........................................................................................................... M80

Werneburg, Brian ...............................................................................T109, W91, W92

West, Jef ........................................................................................................... M131

Westin, Carl-Frederik ............................................................................................W137

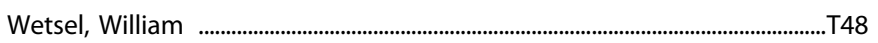

Whelan, Jessica ..........................................................................................................W90

Whelan, Tobias ................................................................................................M41, M140

Whicker, Cady Lauren ................................................................................................ M56

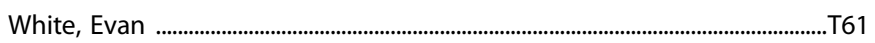

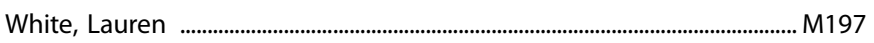

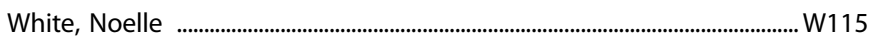

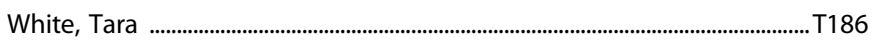

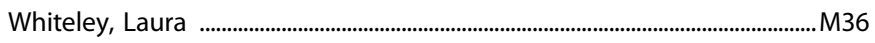

Whiteman, Sarah ......................................................................................................T69

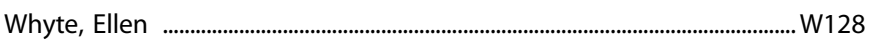

Wichmann, Romy ..................................................................................................... T1 18

Widge, Alik .................................................................................................................9, 9.3

Wiers, Corinde ....................................................M159, W151, W155, W156, W173

Wild, Phillipp S. .......................................................................................................... M92

Wilkinson, Scott .........................................................................................................W96

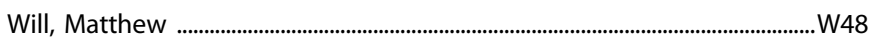

Williams, Aislinn ........................................................................................................... M193

Williams, Alexia ........................................................................................................... T107

Williams, Christopher ................................................................................................ 33

Williams, Leanne ....................................................................... 55, M56, W67, W195

Williams, Maya ..............................................................................................................W148

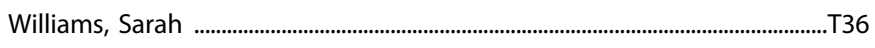

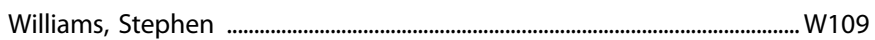

Williamson, David .....................................................................................................W1 18

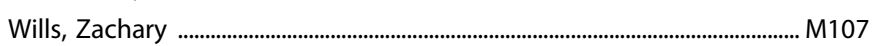

Willuhn, Ingo ................................................................................................................... 46.1

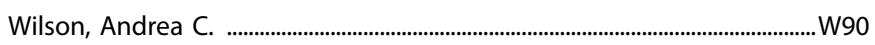

Wilson, Rashaun ............................................................................................................ M18

Wiltshire, Charis ..............................................................................................................W17

Wimmer, Mathieu ........................................................................................................ T178

Winder, Danny .................................................................................................. T38, T55

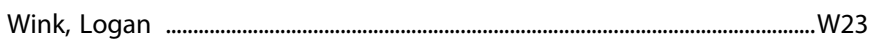


Winters, Sterling

Witt, Emily

..T163

Wohleb, Eric

T120

Wojtas, Ireneusz

Wolf, Daniel

T59, W114

Wolf, Marina

Woller, Sarah

W1

Wolosker, Herman

Wolozin, Benjamin

...T174

Won, Moojun

M47

Wong, Clara

W165

Wong, Dean

...T171

Wong, Jason

M187

Wong, Ma-Li

..T105

Wong, Wan Wa

M103

Woo, Christina

....T36

Woo, T. Wilson

W137

Wood, Daniel .T162, W80

Wood, Elizabeth $\mathrm{K}$ ....T83

Wood, Marcelo

Woodbridge, Hannah ......... M161

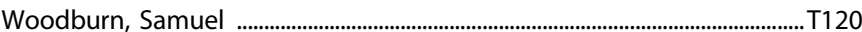

Woodcock, Eric

Woods, Scott

. 8.4, W119

Woodside, Blake

Woodward, Neil M115, M116, T154

Worhunsky, Patrick

M191

Working Group, Schizophrenia ......................................................................... W126

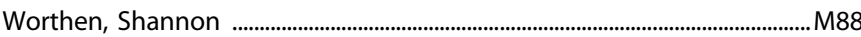

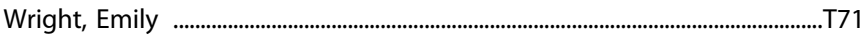

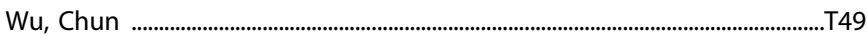

Wu, Lorna ..................................................................................................................T91

Wu, Melody ........................................................................................................................T107

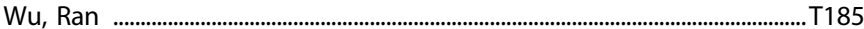

Wu, Renrong .................................................................................................M22, T137

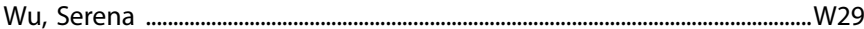

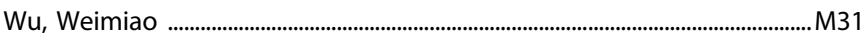

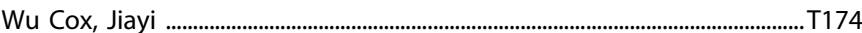

Wulff, Andreas

T41, T192, W97, W99

Wunder, Andreas M105, W65 M88, M89

Wyatt, Ryan

T50, W178

$X i$, Zheng-Xiong

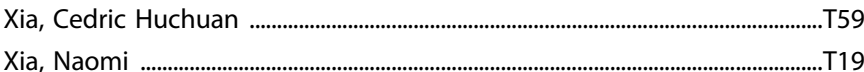

Xia, Yan .................................................................................................................... T141

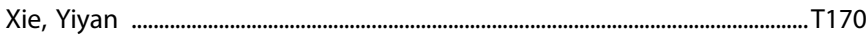

Xiong, Yanyu .....................................................................................................................W48

$\mathrm{Xu}$, Christine ........................................................................................................ T107

$\mathrm{Xu}$, Xiangmin ......................................................................................................W2

Xu, Ying .............................................................................................................................W104

Xue, Joany ..............................................................................................................W153

Yagoda, Sergey ..................................................................................................... M125

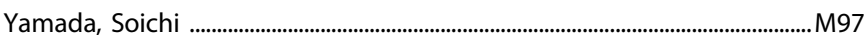

Yamamoto, Jun ................................................................................W131

Yamamuro, Kazuhiko ..................................................................................... M34, W196

Yamauchi, Takahira
Yan, Zhen ..................T40

Yanes-Lukin, Paula K. ............................................................... T128

Yang, Genevieve ...................................................................................... T200

Yang, Hao ......................................................................................................... T101

Yang, Joanna ............................................................................................................. T161

Yang, Kun ..........................................................................................................W8

Yang, Ruirong ...................................................................................................... T127

Yang, Shanna ................................................................................................................... W151

Yang, Yvonne .................................................................................................. T145

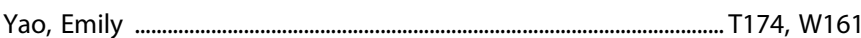

Yarema, Mark .............................................................................................................W73

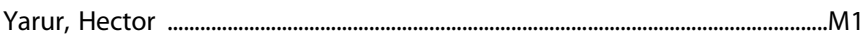

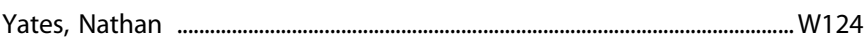

Yates III, John ...........................................................................................................T39

Yavorskyy, Vasyl .................................................................................................... T100

Ye, Kenny ......................................................................................................................... T81

Ye, Qiying ...…………………………………………………………………………... T170

Yee, Joshua .....................................................................................................T52

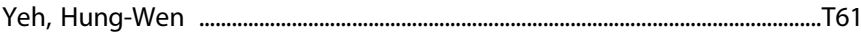

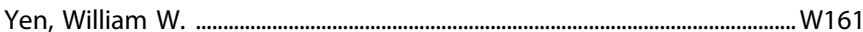

Yin, Jiani ........................................................................................... M111

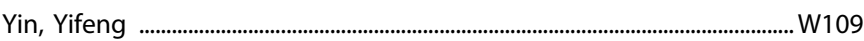

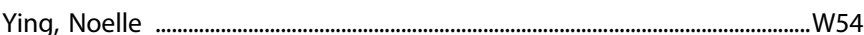

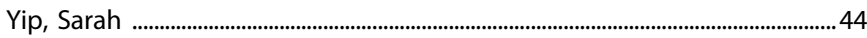

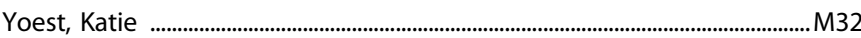

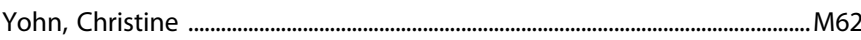

Yokoyama, Sae ............................................................................................................... T107

Yolken, Robert ..........................................................................................................W113

Yonga, Michele-Vera .............................................................................................W156

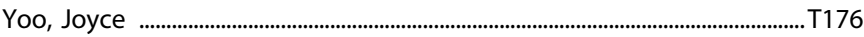

Yorgason, Jordan ...…………………………………………………………………...... M16

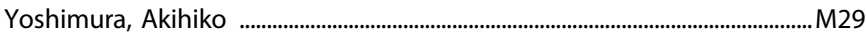

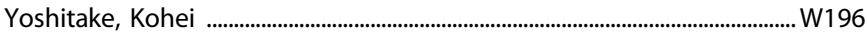

YOu, Zhi-Bing ...…………………………………………………………………….... T179

Young, Amanda ................................................................................................................. T111

Young, Andrea .................................................................................................W50

Young, Jared ........................................................................................................... M198

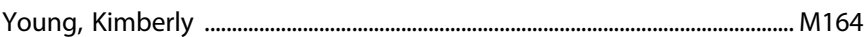

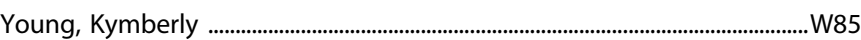

Young, Larry ……………………………………………………………...40, T98, W26

Young, Robert ................................................................................................................M78

Youngstrom, Eric A. ......................................................................................W35, W50

$\mathrm{Yu}$, Bei ..................................................................................................................... M1 $\mathrm{M} 11$

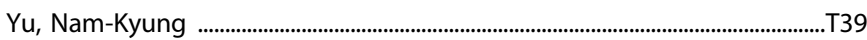

$\mathrm{Yu}$, Qiongru .................................................................................................................. T6

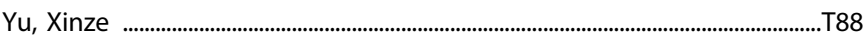

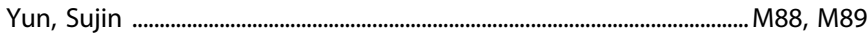

Yurgelun-Todd, Deborah ...................................................................T79, T122

Zabik, Nicole ...................................................................................................................

Zacharek, Sadie J. .......................................................................................................M24

Zachariou, Venetia .................................................................... 32, 32.3, M148, T107

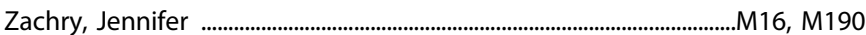

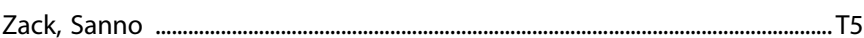

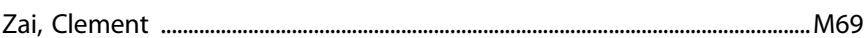

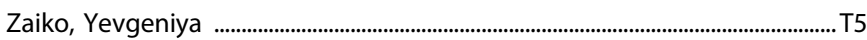

Zakiniaeiz, Yasmin ........................................................................................................W157 


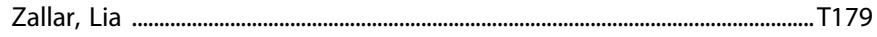

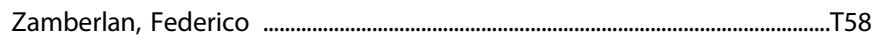

Zammit, Gary ............................................................................................................. M137

Zand, Amanda ........................................................................................................W37

Zand Vakili, Amin ..........................................................................................M10, W86

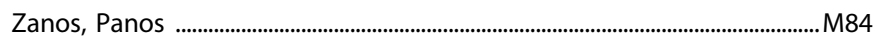

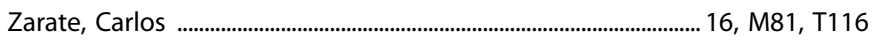

Zarate Jr., Carlos ............................................................................................................M57

Zegers, Juan ............................................................................................................................

Zeilhofer, Hanns U. ......................................................................................................... W8

Zeni, Courtney ........................................................................................................... M108

Zhang, Chunling ..................................................................................................... T141

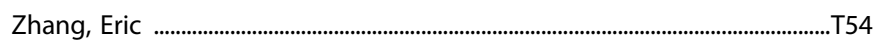

Zhang, Eva Yu ....................................................................................................... M100

Zhang, Fan ...........................................................................................................W3

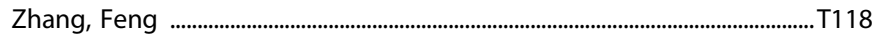

Zhang, Gongliang ...........................................................................................W115

Zhang, Huaibo ................................................................................................M149, T164

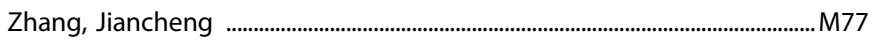

Zhang, Qiaoyi .................................................................................................................T93

Zhang, Rui ..........................................................................................M159, W151, W156

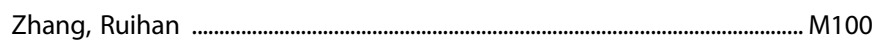

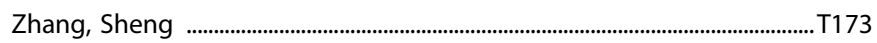

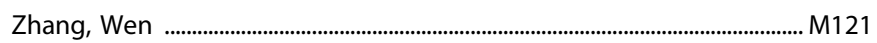

Zhang, Wenchao ......................................................................................................W4

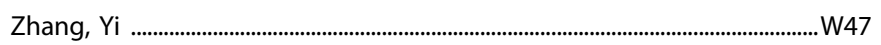

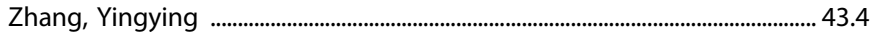

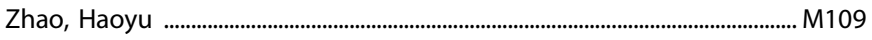

Zhao, Jingping …………………………………………………………...... M22, T137

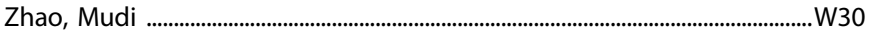

Zhao, Yihua ................................................................................................126, T134

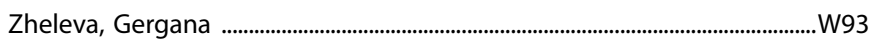

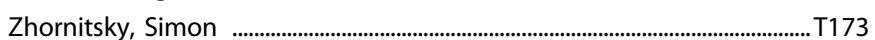

Zhou, Jia .....................................................................................................................M49

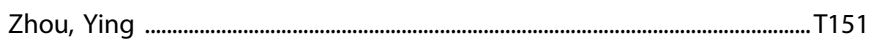

Zhu, Bokai ........................................................................................................... M106

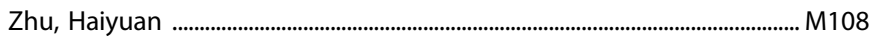

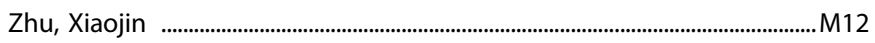

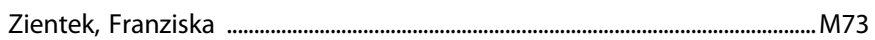

Zigman, Jeffrey ..........................................................................................................T179

Zilverstand, Anna .......................................................................................................T43

Zimmerman, Sara .................................................................................................M44

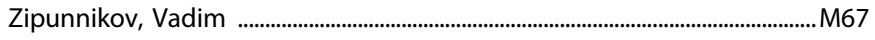

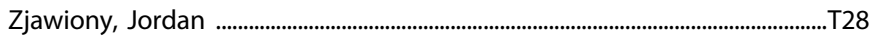

Zlebnik, Natalie ......................................................................................... M180, M18

Zohar, Joseph ............................................................................................................... 18

Zomorrodi, Reza ..............................................................................T25, T153, W107

Zong, Wei ........................................................................................................... W125

Zsido, Rachel ......................................................................................................M73, W93

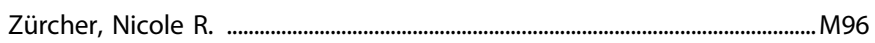

Zweifel, Larry ................................................................................................... T52, T54

Zywiak, William ........................................................................................................... T18 